\title{
Bargaining power in strategic games and economic decision-making
}

Citation for published version (APA):

Britz, V. (2011). Bargaining power in strategic games and economic decision-making. [Doctoral Thesis, Maastricht University]. Universitaire Pers Maastricht. https://doi.org/10.26481/dis.20111216vb

Document status and date:

Published: 01/01/2011

DOI:

10.26481/dis.20111216vb

Document Version:

Publisher's PDF, also known as Version of record

\section{Please check the document version of this publication:}

- A submitted manuscript is the version of the article upon submission and before peer-review. There can be important differences between the submitted version and the official published version of record.

People interested in the research are advised to contact the author for the final version of the publication, or visit the DOI to the publisher's website.

- The final author version and the galley proof are versions of the publication after peer review.

- The final published version features the final layout of the paper including the volume, issue and page numbers.

Link to publication

\footnotetext{
General rights rights.

- You may freely distribute the URL identifying the publication in the public portal. please follow below link for the End User Agreement:

www.umlib.nl/taverne-license

Take down policy

If you believe that this document breaches copyright please contact us at:

repository@maastrichtuniversity.nl

providing details and we will investigate your claim.
}

Copyright and moral rights for the publications made accessible in the public portal are retained by the authors and/or other copyright owners and it is a condition of accessing publications that users recognise and abide by the legal requirements associated with these

- Users may download and print one copy of any publication from the public portal for the purpose of private study or research.

- You may not further distribute the material or use it for any profit-making activity or commercial gain

If the publication is distributed under the terms of Article $25 \mathrm{fa}$ of the Dutch Copyright Act, indicated by the "Taverne" license above, 
Bargaining Power in Strategic Games and Economic Decision-Making 
Bargaining Power in Strategic Games and Economic Decision-Making

(C) Copyright Volker Britz, Maastricht 2011

All rights reserved. No part of this publication may be reproduced, stored in a retrieval system, or transmitted, in any form, or by any means, electronic, mechanical, photocopying, recording or otherwise, without the prior permission in writing from the author.

This book was typeset by the author using $\mathrm{LT}_{\mathrm{EX}}$.

Published by Universitaire Pers Maastricht

ISBN: 9789461591005

Printed in The Netherlands by Datawyse Maastricht 


\title{
BARGAINING POWER IN STRATEGIC GAMES AND ECONOMIC DECISION-MAKING
}

\author{
DISSERTATION \\ to obtain the degree of Doctor at \\ Maastricht University, \\ on the authority of the Rector Magnificus, \\ Prof. dr. G.P.M.F. Mols, \\ in accordance with the decision of the Board of Deans, \\ to be defended in public
}

on Friday, December 16, 2011, at 14.00 hours

by

Volker Britz

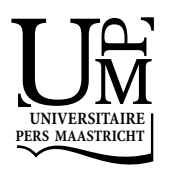




\section{Supervisor:}

Prof. dr. P.J.J. Herings

\section{Co-supervisor:}

Dr. A. Predtetchinski

\section{Assessment Committee:}

Prof. dr. H.J.M. Peters (chairman)

Prof. dr. J. Davila, Paris

Dr. A. Perea

The research for this dissertation was financially supported by the Netherlands Organization for Scientific Research (NWO). 


\section{Acknowledgements}

On the face of it, this dissertation is the product of five years of research done as a Ph.D. student. At the same time, for me personally, it also marks the end of nine years spent in Maastricht. This has been a long and formative time period. Looking back, I am glad to say that I do not regret a single day.

It is impossible to address all those who have made a difference to me throughout the years. Therefore, what I write in acknowledgement is certainly not all that I feel - but all that I do write is sincerely felt.

First of all, I would like to thank my promotor and co-promotor, Prof. P. Jean-Jacques Herings and Dr. Arkadi Predtetchinski for their guidance. From the first to the last day of my Ph.D. project, Arkadi and Jean-Jacques have been very generous, consistent, and patient in giving their advice, encouragement, and support. Neither Arkadi nor Jean-Jacques have ever limited themselves to pouring red ink on my countless draft versions. They have let me share in and benefit from their impressive knowledge, insight, and experience.

Some other members of the faculty have played a special role in my development, knowing me since I was a first-year undergraduate student and encouraging me to begin a research career: Ronald Peeters and Martin Strobel. Moreover, I would like to thank Prof. Julio Davila and Dr. Andrés Perea for being part of the assessment committee and Prof. Hans Peters for being its chairman.

I have enjoyed the company of many colleagues in the Economics department and especially of the other Ph.D. candidates with whom I could share experiences and have fun during and after work (and, of course, in the sometimes lengthy lunch breaks). In particular, I have had the honor to be the paranimf of Helga Habis, and I am very grateful to Franziska Tausch and Eva Wölbert for their friendship and for being my paranimfen.

The department secretaries, Nicole Hulsman and Elke Lucas, have given me a lot of valuable support - in particular with regard to the many administrative hurdles and procedures involved in my job market activities at the end of my project.

I have studied and worked hard in Maastricht, but I have also played (although, on second thought, I might have played a bit harder ...). I have enjoyed being part of some courses and activities of the students' drama association Alles is Drama. Kiki, Lennart, and Lieneke have been patient and skillful teachers. I have very much enjoyed my small moments in the limelight, and there might also have been beneficial side-effects on my conference and seminar presentations.

I have taken part in many activities of Ph.D. Academy (a.k.a. Provum) which have brought me in touch with a lot of Ph.D. students from other departments and faculties. Naturally, doing a Ph.D. requires specializing in a very narrow field for several years. So, it has been 
reassuring and pleasant to socialize with people in the same situation, but often from completely different fields. Fortunately, many people involved with Ph.D. Academy share my interest in theatre. In particular, Jan, Jasper, and Jessie have taken the initiative to organize the acting and improvisation evenings which have been a welcome (and challenging) distraction from my research.

The student chaplaincy Tafelstraat 13 has played a very special role during my time in Maastricht. In terms of social student life, I took quite some time to look for my place, but here I found it. In many different situations throughout the years, when I was happy or sad, full of hopes or full of doubts, or just hungry, I have felt welcome and at home here. There are more people to thank for this than I can possibly name: members of the team and members of the plein, cooks and helpers, regulars and visitors. Representative of all of them, I would like to thank Berenike and Julia who have been the chaplaincy's backbone for the last few years - and Elleke, who has quite literally opened the door of Tafelstraat to me for the very first time.

Finally, I would like to thank my parents, Wolfgang and Dagmar Britz, for their enduring love and support. Throughout my early years, my parents have done a lot of things for me which do not seem so unlike those you do when writing a paper - taken care of countless subtle details, trusted their intuitions, overcome the setbacks, celebrated the breakthroughs, and shelved some unfavourable referee reports. Pushing the analogy a little bit further, my grandparents would surely deserve more than a footnote for the advice and support which they have contributed. In the meantime, of course, this analogy has become somewhat obsolete. Unlike the lines we write for the papers, I have developed a life of my own, with friends, interests, and a job which my parents know only from afar. Yet it remains a blessing that they continue to follow the latest developments with interest, sometimes lending a hand, but always keeping their fingers crossed. 


\section{Contents}

$\begin{array}{ll}\text { Acknowledgements } & 5\end{array}$

1 Introduction $\quad 9$

1.1 The surplus-division problem and Rubinstein bargaining . . . . . . . . . . . . 9

1.2 Proposal Power . . . . . . . . . . . . . . . . . . . . . . . . . 9 9

1.3 Commitment power . . . . . . . . . . . . . . . . . 12

1.4 Theory of the firm . . . . . . . . . . . . . . . . . . 14

2 Non-cooperative support for the asymmetric Nash Bargaining Solution 17

2.1 Introduction . . . . . . . . . . . . . . . . . . . . . . . 17

2.2 The Bargaining Game . . . . . . . . . . . . . . . . . . . 20

2.3 Analysis of Stationary Equilibrium . . . . . . . . . . . . . . . . . . . 21

2.4 The Limit Equilibrium . . . . . . . . . . . . . . . . . . . . . . . . . . 25

2.5 Conclusion . . . . . . . . . . . . . . . . . . . . 32

3 Optimal value commitment in bilateral bargaining 33

3.1 Introduction . . . . . . . . . . . . . . . . . . . . . . . 33

3.2 The game with one committed player . . . . . . . . . . . . . . . . 35

3.3 Subgame perfect bargaining equilibrium . . . . . . . . . . . . 36

3.4 Optimal commitment . . . . . . . . . . . . . . . . . . . . 42

3.5 Bargaining with two committed players . . . . . . . . . . . . . . 47

3.6 Optimal commitment for two players . . . . . . . . . . . . . . . . . . 51

3.7 Conclusion . . . . . . . . . . . . . . . . . . . . . . 58

4 Theory of the firm - Bargaining and competitive equilibrium 59

4.1 Introduction . . . . . . . . . . . . . . . . . . . . . . . 59

4.2 Model Description . . . . . . . . . . . . . . . . . . . . 61

4.3 Reduced Form Bargaining Game . . . . . . . . . . . . . . . . . . . . . . . . . . 64

4.4 Weighted Nash Bargaining Solution . . . . . . . . . . . . . . . . 70

4.5 Producer Choice . . . . . . . . . . . . . . . . . . . . . . 74

4.6 Conclusion . . . . . . . . . . . . . . . . . . . . . 82

$\begin{array}{lr}\text { Summary } & 85\end{array}$

$\begin{array}{ll}\text { Nederlandse Samenvatting } & 87\end{array}$

$\begin{array}{lr}\text { References } & 89\end{array}$

$\begin{array}{ll}\text { Short Curriculum Vitae } & 93\end{array}$ 



\section{Introduction}

\subsection{The surplus-division problem and Rubinstein bargaining}

The main theme of this dissertation is bargaining power. Strategic sources of bargaining power are addressed as well as its importance in a particular context of economic decision-making. The topic is treated from the angle of microeconomic theory and, in particular, non-cooperative game theory.

The basic economic problem which underlies the dissertation is the division of gains from cooperation. There are a number of players who can create or access some surplus. However, each individual player can only consume any of this surplus once all the players have agreed on how the surplus should be divided among them.

One approach which economic theorists have taken to this problem is to assume that the parties involved engage in a formal bargaining process in order to find an agreement. This bargaining process can then be modeled as a non-cooperative game and analyzed with the relevant game-theoretic tools.

A seminal paper by Rubinstein (1982) has inspired a rich literature on bargaining games. In a typical model of Rubinstein bargaining, the bargaining process is subdivided into rounds, each of which has the following structure. First, one particular player is designated as the proposer according to some rule (the protocol). Second, the proposer suggests a particular division of the surplus. Third, the other players accept or reject this proposal. If a proposal has been approved by all players, then the surplus is divided according to this proposal and the game ends. If, however, a proposal is not accepted by all players, then a new round of bargaining starts. However, the disagreement and ensuing delay to the next round comes at a cost. This cost is either represented in the model as discounting future consumption in the players' utility functions, or as an exogenously given risk that negotiations break down for good after the rejection of a proposal. In either case, the cost of delay is often referred to as bargaining friction.

The first two chapters of the dissertation deal with sources of strategic power in a Rubinstein bargaining situation. In the first chapter, I study the role of the bargaining protocol (that is, the rule determining which player proposes in which round) as a source of bargaining power. In the second chapter, I study a situation where players can make commitments before they come to the bargaining table, and I am interested in the role of the ability to commit as a source of bargaining power. In the third chapter, I apply the insights gained on surplus division by bargaining to a situation where a number of shareholders jointly own a firm and need to agree (through a bargaining process) on the choice of a production plan for their firm.

\subsection{Proposal Power}

One of the crucial ingredients in a Rubinstein bargaining model is the protocol which determines the proposer in each round of bargaining. The specification of a protocol is a very important modeling choice since the right to make proposals is arguably the most recognized and discussed 
source of strategic power in bargaining games. It can be interpreted as corresponding to the common sense idea that the "right to set the agenda" confers power in a bargaining situation.

Shaked and Sutton (1984) have concisely pointed out that in Rubinstein bargaining a player's payoff "coincides with the sum of the shrinkages" of the surplus which occur while that player is the proposer. Before addressing the contribution of my dissertation, I will briefly summarize the intuition underlying Shaked and Sutton's statement. To this end, suppose that two players, Miss One and Mister Two, bargain about the division of a surplus of size one. If they do not agree in the current round, then only a part of the surplus will be left on the table in the next round, while the rest will be lost - indeed, delay is costly. Let Miss One be the proposer in the current round. Why would Mister Two ever reject Miss One's proposal? He would do so only if that proposal gives him less of the surplus than what he hopes to get in the sequel of the bargaining process (his continuation payoff). Clearly, this amount can never exceed that which will be left in the next round. Suppose for the sake of the argument that Miss One can slice up the surplus into two pieces, namely the one which will disappear if no agreement is reached in the current round, and the one which will be left on the table in the next round. In order to give Mister Two his continuation payoff, she has to use only (part of) the piece which will remain in the next round. Conversely, she can appropriate the piece which would disappear for herself. Since the same argument can be repeated for each round of bargaining, a player's payoff will indeed correspond to the sum of all those pieces of the surplus which would disappear over time if her proposals were to be rejected.

With this intuition in mind, the main result of Chapter 2 should have some natural appeal. It says the following.

If the degree of bargaining friction is sufficiently small, then a player's bargaining power is given by the frequency with which this player expects to be the proposer in the long-run.

I will now provide some more detail on the model in Chapter 2, and briefly discuss a number of technical issues. On the basis of this discussion, I will then be able to restate the main result in a more precise way.

The model in Chapter 2 of the dissertation is one where some finite number of players need to choose one payoff allocation from a set of feasible allocations. Players are assumed to be expected utility maximizers, but utility does not need to be transferable among them. The bargaining friction is embodied in an exogenous risk of breakdown at each instance of disagreement. The bargaining protocol is such that proposers are selected through a Markov process. That is, in each round the proposer is drawn from some probability distribution, and which probability distribution this is in a particular round depends solely on the identity of the player who proposed in the previous round. We impose assumptions on this Markov process which guarantee that it has a unique stationary distribution. Loosely speaking, this means that the probability with which a player expects to be the proposer in the long-run is independent of the identity of the current proposer.

The bargaining protocol based on the Markov process generalizes several protocols which are well-established in the bargaining literature. One such protocol is the one where the proposer in each round is drawn from one and the same probability distribution, see for instance Miyakawa 
(2008). This case corresponds to a Markov process in which all rows of the transition matrix are identical. Another well-known protocol involves a fixed order of proposers. That is, the proposers are selected one after the other in a deterministic way. This case corresponds to a Markov process for which the transition matrix is a permutation matrix. It can be seen as a natural extension of Rubinstein's (1982) alternating offer protocol to the case with more than two players.

The solution concept applied to the game in Chapter 2 of the dissertation is the subgameperfect Nash equilibrium in stationary strategies. In the aforementioned bargaining model with two players due to Rubinstein (1982), the standard subgame-perfect equilibrium concept uniquely predicts a payoff allocation. However, it is well-known in the literature that this uniqueness is lost when one extends the model to more than two players (at least if one is interested in unanimous agreement among all players at once). In order to narrow down the set of equilibrium payoffs, many authors have restricted attention to those subgame-perfect equilibria which are in stationary strategies. In the context of Rubinstein bargaining, a stationary strategy has a player make the same proposal whenever she is the proposer. Moreover, whether a player accepts or rejects a proposal under a stationary strategy depends only on the proposal (and the proposer).

The Nash bargaining solution is a well-known solution concept in bargaining theory. It chooses a particular element of a set of feasible payoff allocations by maximizing the product of the gains which players make relative to a disagreement point. Nash (1953) shows that this solution is unique in satisfying a number of axioms: Symmetry, Invariance, Pareto-optimality, and Independence of Irrelevant Alternatives. The asymmetric Nash bargaining solution is a generalization of the Nash bargaining solution in which the payoffs of different players may have different weights. Mathematically, the asymmetric Nash bargaining solution is found by maximizing a product of players' payoffs where each factor is raised to some power. If the exponent is the same across players we have the standard Nash bargaining solution.

In Chapter 2, the asymmetric Nash bargaining solution is obtained as the payoff allocation corresponding to subgame-perfect equilibria in stationary strategies. A more formal statement of the main result is the following.

In the limit as the risk of breakdown goes to zero, all subgame-perfect equilibria in stationary strategies lead to the payoff allocation which corresponds to the asymmetric Nash bargaining solution with bargaining weights given by the stationary distribution of the Markov process.

As pointed out before, the assumption that the selection of the proposer follows a Markov process admits two special cases which have some prominence in the literature. The first case is where the proposer in each bargaining round is drawn from one and the same probability distribution on the players. For this case, the result in Chapter 2 implies previous findings by Hart and Mas-Colell (1996), Miyakawa (2008) and Laruelle and Valenciano (2008). The second case is where players propose in a fixed order. For that case, our result implies the previous finding by Kultti and Vartiainen (2007). The result provided in Chapter 2 unifies a number of existing results in the literature and substantially generalizes them. 


\subsection{Commitment power}

While Chapter 1 of the dissertation has focussed on strategic power derived from the protocol, we now consider pre-bargaining commitment as a source of power.

In a game-theoretic context, a commitment is a way in which a player can rule out some possible course of action which he might otherwise have taken in the future. Differently put, a commitment is a way in which a player can "tie his own hands" to obtain a strategic advantage. Schelling (1960) has highlighted the importance of commitment power in bargaining.

"The power to constrain an adversary may depend on the power to bind oneself; [ ...] in bargaining, weakness is often strength, freedom may be freedom to capitulate, and to burn bridges behind one may suffice to undo the opponent."

In the bargaining context of this dissertation, a commitment should be thought of as a credible announcement by a player before the bargaining process not to enter into certain agreements.

One very well-known illustration of pre-bargaining commitment is mentioned by Schelling (1956). In this example, a firm and a union are about to bargain about the future wage of the workers. Before bargaining starts, the negotiators who are going to represent the union at the bargaining table address the crowd of workers with a dramatic announcement to the effect that they will never settle for less than a certain wage. This announcement is observable by the firm's management. The idea is that the union negotiators can now claim at the bargaining table that their hands are tied so as not to accept less than the wage they had announced to the workers. After all, if they were to settle for less, these workers would live out their disappointment and treat their negotiators in a number of unpleasant ways, such as unseating the union leadership, throwing tomatoes, etc. In a more game-theoretic terminology, the ability to raise expectations and arouse emotions among the workers gives the negotiators the chance to make credible threats at the bargaining table.

One important problem is to decide how committed a player can be. To see where the problem lies, suppose that we want to assume that before bargaining, one player can indeed make a commitment not to settle for less than some amount. Assume furthermore that this commitment is indeed perfect, that is, it is binding and observable by the opponent. In that case, the power of commitment totally overrules any form of power derived from the actual bargaining situation. The strategic situation resembles that in the well-known ultimatum game, and the committed player can receive the entire surplus in equilibrium. In Schelling's (1960) original terms, the ability to make a perfect commitment allows a player to "squeeze the range of indeterminacy down to the point most favorable to him".

If we would like to assume instead that both players can simultaneously make a prebargaining commitment, then we create a strategic situation similar to that in a version of the Nash demand game (Nash 1953) without noise: Any (efficient) division of the surplus could be supported by an equilibrium. The conclusion is that perfect pre-bargaining commitments lead either to an extreme prediction or no prediction at all on the allocation of the surplus. The literature has developed somewhat weaker notions of commitment. For instance, both 
making and breaking the commitment may come at a cost. It may cost a player something to make a commitment credible, or a player may be able to revoke a standing commitment at some (non-prohibitive) cost (Muthoo 1992, Muthoo 1996). Another approach has been the introduction of an imperfect information framework in which players are uncertain about each other's rationality (Abreu and Gul 2000, Kambe 1999). The approach which I have taken in the dissertation is different from the existing literature. I have taken the notion of perfect commitment as a starting point and tried to limit the degree to which commitment is "perfect" - not by writing a more sophisticated model but by imposing two restrictions on commitment which I find rather mild and naturally appealing. The two restrictions which I impose on the ability to commit can be summarized intuitively as follows.

1. One cannot commit to what one does not care about.

2. One cannot remain committed to what has become impossible.

I discuss both restrictions in turn. In the existing literature on commitment in bargaining, it is often the case that commitments are expressed in terms of a percentage share of the surplus to be divided. At the same time, however, it is assumed that the players care about the discounted present value of their consumption. Hence, the players are assumed to be committed in terms which are different from those from which they derive utility. Li (2007) has suggested that it would be more credible to let players express their commitments in those terms which also enter the utility function, and he has called this approach value-commitment as opposed to the aforementioned share-commitment. The first of the restrictions which I have imposed is in the spirit of Li (2007) in that it means that players make value-commitments. I assume that the available surplus shrinks over time and that players commit prior to bargaining to some amount (rather than a share) of that surplus. Consequently, when disagreement lasts long enough, the size of the surplus will eventually shrink below the amount of the commitment. Now, the second restriction above means that player 1's commitment is no longer credible and thus no longer binding from that point onwards. The two restrictions on commitment create a trade-off for the player. Bold commitments lose their binding power soon, while milder commitments remain in force longer. This makes it intuitively clear that is no longer optimal to commit to the entire surplus.

I study three versions of this game: one in which only one player makes a pre-bargaining commitment, and the cases in which both players can do so either simultaneously or sequentially. For each case, I devise a solution concept which is a slight refinement of subgame-perfect Nash equilibrium. When the discount factor is sufficiently small, I obtain results which are similar to those in the case of perfect commitment. That is, in equilibrium the committed player obtains the entire surplus if only one player can commit, and (almost) every efficient allocation is an equilibrium if both players can commit. In the limit as the discount factor becomes large, however, these results substantially change. One noteworthy result is that if the proposal power is concentrated in one player while the other player can commit then the division converges to the equal split. In this particular sense, proposal power and commitment power are equally important determinants of the bargaining outcome. 
In the case where both players make simultaneous commitments prior to the bargaining process, I find a range of efficient surplus divisions which can be supported by equilibria. If the discount factor is large, this range consists of at most one fifth of the range of feasible efficient divisions. The endpoints of the range are determined by the recognition probabilities. The equal split is the unique division which is an equilibrium for any values of the discount factor and the recognition probabilities.

\subsection{Theory of the firm}

Chapters 2 and 3 address sources of strategic power in bargaining. In the final chapter of the dissertation, the idea is to apply bargaining theory to the problem of decision-making within the firm when financial markets are incomplete.

Suppose that a firm is jointly owned by several shareholders. This firm exists in an environment with two time periods (today and tomorrow) in which there is uncertainty about the future. Differently put, the owners of the firm are aware today that one out of many states of the world is going to realize tomorrow but neither do they know which one, nor do they necessarily have a common belief on the probability of the states. Facing this uncertainty, the owners have to decide on a production plan for the firm. That is, there is a set of productive activities which the firm could undertake, one of which has to be chosen jointly by the owners. A production plan can be thought of as a specification of an input or output today, and an input and output for each possible state of the world tomorrow.

It can be the case that the different owners of the firm have different preferences over consumption today and in the different possible states of the world tomorrow. For example, one owner may be more patient than the other and therefore find consumption today relatively less important. Moreover, one owner may believe with a very high probability that a particular state of the world will occur tomorrow and will therefore find the consumption in that state very important. Another owner may have a different belief about the future and thus different preferences over consumption in those states.

How can the owners come to an agreement? In economic theory, the easiest way to deal with this problem is to assume the existence of complete financial markets. When financial markets are complete, it is possible for the agents in an economy to trade off consumption across time and across all states of the world. But when trades are possible across time and states, then this effectively creates prices for consumption in each state. As a result, all agents can agree on maximizing the profit of the firm. They can then split this profit and individually access the financial markets in order to purchase they most desirable consumption bundle they can afford.

This approach breaks down, however, in a world of incomplete financial markets. It is well-known in the literature that when some or all asset markets are missing, the different shareholders of a firm tend to have a conflict about the choice of a production plan. How should this conflict be dealt with in a formal economic model? The existing literature has often taken a normative approach to the question: Based on the idea of constrained Paretooptimality, Drèze (1974) and Grossman and Hart (1979) have designed criteria for the choice of 
a production plan which rely, loosely speaking, on a weighted average of the owners' valuations of consumption in different states. These criteria form the basis for the standard equilibrium concepts in the literature on incomplete markets. As pointed out in the authoritative textbook of Magill and Quinzii (1996), those equilibrium concepts suffer from the drawback that they are not as yet supported by an explicit bargaining process by which the owners would choose a production plan satisfying some normative criterion. The main contribution of Chapter 4 is to develop and analyze such a bargaining process. The results derived from this model are only partially in line with standard economic theory.

The bargaining process used in Chapter 4 is similar to Rubinstein bargaining as described earlier. The proposer is selected from the same probability distribution in each round of bargaining, and unanimous approval is required for a proposal to pass. The time horizon is potentially infinite but there is an exogenous risk of breakdown after each instance of disagreement. As pointed out before, this protocol is a special case of the protocol analyzed in Chapter 2. The main difference between the bargaining process in Chapter 4 and Rubinstein bargaining is that a proposal in this context does not consist of a payoff allocation but rather of a production plan and a scheme of transfers, that is, side-payments among the owners of the firm which are expressed in terms of consumption today. It is demonstrated that under some assumptions there is a one-to-one correspondence between the problem of choosing a production plan (plus a scheme of transfer payments) and the problem of choosing a payoff allocation from a set of feasible allocations as in Chapter 2. Put another way, it is shown that the economic problem discussed in Chapter 4 can be seen as an instance of the abstract problem analyzed in Chapter 2. While Chapter 2 assumes that some abstract set of feasible payoff allocations has certain properties, it turns out in Chapter 4 that the problem of decision-making within the firm under incomplete financial markets gives rise to a set of feasible payoff allocations which does have those same properties.

In the special case where markets are complete, the equilibrium of the bargaining model in Chapter 4 does involve the profit-maximizing production plan. However, the generated profits need not be distributed to the owners according to their shares of ownership alone. In fact, redistribution through transfers does occur generically in the initial endowments which parameterize the economy and in the distribution of bargaining power among the owners (which need not be identical with the shares of ownership). Hence, compared to standard economic theory, the bargaining model in this dissertation predicts the same production plan but not the same payoff allocation in the case with complete markets. In the case with incomplete markets, the bargaining model makes predictions about the choice of a production plan and the concomitant payoff allocation which are generically different from standard theory. 



\section{Non-cooperative support for the asymmetric Nash Bargaining Solution}

\subsection{Introduction}

This paper ${ }^{1}$ contributes to the Nash program of supporting solution concepts from cooperative game theory by obtaining them as equilibrium outcomes of suitably constructed non-cooperative games. More specifically, we will be concerned with the asymmetric Nash bargaining solution. Consider a situation where two players receive a given pair of payoffs if they disagree, but may obtain any element of a convex set of other (superior) payoff pairs if they mutually agree on one such element. The Nash bargaining solution (NBS) is that payoff pair which maximizes the product of players' gains over their disagreement payoff. Nash (1950) showed that this is the unique bargaining solution satisfying the axioms of scale invariance, symmetry, efficiency, and independence of irrelevant alternatives. One can generalize the NBS by assigning different weights to the players. The asymmetric Nash bargaining solution (ANBS) is that payoff pair which maximizes a weighted product of players' gains over their disagreement payoff, see Kalai (1977).

The ANBS is used to gain insights on a wide variety of problems in economics. For instance, Bester (1993) compares the effects of different pricing mechanisms on price and quality of a product. In particular, posted pricing is compared to bargaining between a buyer and a seller. In the latter case, the ANBS is taken to be the outcome of the bargaining interaction.

Another common application is wage bargaining between a firm and a union: Firm owners and workers can agree to produce and hence create a surplus. A part of the surplus goes to the workers as their wage, and the rest goes to the shareholders. If, however, the two sides cannot find an agreement, the workers may strike or the firm may shut down so that no surplus is generated. In a seminal paper on wage bargaining, Grout (1984) studies the effect of different legal frameworks on wage bargaining. Throughout the analysis, it is assumed that bargaining between the firm and the union leads to the outcome predicted by the ANBS.

The prominent use of the ANBS in applications highlights the need for strong noncooperative underpinnings of this concept. In the case of the ANBS, it is imperative to examine the non-cooperative or strategic sources of players' "bargaining power" which is borne out in the weight vector of the ANBS. Nash (1953) presents a non-cooperative demand game with two players who are uncertain about which payoff pairs are feasible. In the limit as the uncertainty vanishes, equilibrium payoffs converge to those predicted by the NBS. Carlsson (1991) takes a similar approach, but with a different source of uncertainty: While the set of feasible payoffs is known to both players, their actions are subject to noise. If players make demands which do not exhaust the available surplus, the remainder is distributed according to an exogenously fixed rule. In the limit as the noise vanishes, there is a unique efficient equilibrium. The payoff

\footnotetext{
${ }^{1}$ This chapter corresponds to the paper V.Britz, P.J.J.Herings, and A.Predtetchinski (2010), "Noncooperative support for the asymmetric Nash Bargaining Solution", Journal of Economic Theory, 145, 19511967.
} 
pair is a particular ANBS; the bargaining weights are determined by the exogenous division rule.

In a seminal paper, Rubinstein (1982) provides a non-cooperative game in which two players negotiate on the division of a pie. The players take turns acting as the proposer. The division of the pie in the unique subgame perfect equilibrium depends upon how strongly players prefer current over future payoffs. In the limit as players become perfectly patient, the equilibrium division converges to the NBS. In their discussion of cooperative and non-cooperative approaches to bargaining, Binmore, Rubinstein and Wolinsky (1986) obtain the NBS in the limit if either players' impatience or the risk of an exogenous breakdown of the negotiations is vanishing.

Although the relationship between cooperative and non-cooperative approaches to bargaining are well understood for the case of two players, such is far less the case when more than two players are involved in the negotiation process. While it is straightforward to generalize the ANBS to $n$ players, the extension of its non-cooperative justification has turned out to be a much more difficult problem. Krishna and Serrano (1996) make use of Lensberg's (1988) stability (consistency) property. They design a non-cooperative bargaining protocol in which players can exit after partial agreements. This game has a unique subgame perfect equilibrium and the payoffs implied by that equilibrium converge to the NBS as the discount factor goes to one. Chae and Yang (1994) obtain uniqueness of perfect equilibrium and convergence to the NBS in a game where a proposer negotiates with one responder at a time. In both papers, the results come at the cost of allowing partial agreements, rather than requiring unanimous consent to a comprehensive proposal.

For the case with unanimous consent, an early support result for the NBS in the $n$ player case is implied by the analysis of Hart and Mas-Colell (1996). More recently, support results for the ANBS have been given by Miyakawa (2008) and Laruelle and Valenciano (2008). All these papers consider a bargaining game where the proposer in each period is drawn from an invariant probability distribution. The stationary equilibrium payoffs turn out to converge to the ANBS with that probability distribution as the weight vector. Our analysis covers this result as a special case. Another special case is a fixed order of proposers, as analyzed by Kultti and Vartiainen (2007), who also show that differentiability of the payoff set's Pareto frontier is essential for the convergence result if there are at least three players.

Other strands of the bargaining literature consider much more general bargaining protocols. For instance, in their analysis of uniqueness and efficiency of equilibria in bargaining games, Merlo and Wilson (1995) assume that both the size of the cake to be divided and the order in which players propose and respond follow a Markov process. Kalandrakis (2004) examines no-delay equilibria in stationary strategies under a Markov selection protocol, where agreement does not necessarily require unanimous consent of all players, but only of those within a winning coalition. The set of such equilibria is characterized and shown to be non-empty. Herings and Predtetchinski (2007) study a game with Markov recognition probabilities, where the set of alternatives is one-dimensional. While studying delay or inefficiencies in bargaining games, other authors have used proposer protocols following stochastic processes, see for instance Cho and Duggan (2005) and Hyndman and Ray (2007) . In these papers, the stochastic process 
is not even required to have the Markov property, so the choice of the proposer may depend on aspects of history other than the identity of the previous proposer. Since more general selection protocols are used in much of the bargaining literature, we find it important to extend this approach to the support results for the ANBS.

In this paper we take a general approach towards multilateral bargaining. We aim at results for the case with $n$ players, a general set of feasible payoffs, and a general bargaining protocol. An informal description of the game we consider in this paper is as follows.

In the first period of an infinitely repeated bargaining game, the identity of the proposer is completely arbitrary. In each subsequent period, one out of the $n$ players is recognized as the proposer according to an irreducible Markov process. Upon recognition, the proposer offers a particular element of a convex and comprehensive set of feasible payoffs. If all players accept the offer, it is implemented. If a player rejects the offer, with some exogenously given and constant breakdown probability the game ends, whereas with the complementary probability the next period starts.

We show that subgame perfect equilibria in stationary strategies exist and we characterize the set of such equilibria. We then study the limit of an arbitrary sequence of such equilibria corresponding to a sequence of vanishing breakdown probabilities. We show that in the limit all players make the same proposal. Our main result is that in the limit this common proposal coincides with the ANBS with the stationary distribution of the Markov proposer selection process as the weight vector. Hence, equilibrium payoffs depend only on the set of feasible payoffs and the stationary distribution associated with the matrix of transition probabilities.

The proof of our result goes well beyond mere technical generalizations of existing proof strategies. Since the reservation payoff of a responding player depends on the identity of the current proposer, reservation payoffs cannot be expressed by a single vector, but correspond to a matrix. For any value of the exogenous breakdown probability, we consider the vectors corresponding to the difference between the equilibrium proposals of a pair of players. We show that in the limit as the breakdown probability vanishes, these vectors span an $(n-1)$ dimensional supporting hyperplane to the set of feasible payoffs at the point corresponding to the common limit proposal of the players. Finally, we demonstrate that the unique normal vector to this supporting hyperplane is proportional to the gradient of the asymmetric Nash product with weights equal to the stationary distribution associated with the matrix of transition probabilities.

Our analysis includes two very frequently encountered proposer protocols as special cases. First, consider the protocol where the proposer in each round is drawn from the same probability distribution. This corresponds to a Markov chain where all the rows of the transition matrix are the same. Our result implies that stationary equilibrium payoffs converge to the ANBS with the time-invariant probability distribution as bargaining weights. Consequently, our findings cover the support results of Hart and Mas-Colell (1996), Miyakawa (2008), and Laruelle and Valenciano (2008). Second, suppose that players make proposals in some fixed order, which is a straight-forward $n$-player extension of the alternating-offer protocol in the classical paper by Rubinstein (1982). The fixed order of proposers induces a stationary distribution of the 
Markov chain with equal weights for all players. The limit equilibrium then corresponds to the NBS, the result shown by Kultti and Vartiainen (2007). In the current paper, we reveal how the aforementioned results are instances of a much more generally valid principle.

We assume that the set of feasible payoffs is comprehensive from below and that all weakly Pareto-efficient payoffs are also strongly Pareto-efficient, implying that the relevant bargaining space is $(n-1)$-dimensional. Herings and Predtetchinski (2007) consider the same proposer selection protocol as in this paper, but study the case of a 1-dimensional set of alternatives where players have utility functions that are linear in the distance to their most preferred alternative. A unique prediction for the equilibrium payoffs is obtained in the limit as the discount factor goes to one. The equilibrium alternative of the bargaining game is contrasted with the prediction of both the median voter theorem and the ANBS and it is argued that there are no general relationships. Full dimensionality of the space of payoffs is therefore crucial to obtain convergence to ANBS.

The paper is organized as follows. Section 2 gives the formal description of the bargaining game and the definition of the equilibrium concept. In Section 3, we give necessary and sufficient conditions for a profile of stationary strategies to be an equilibrium in accordance with that concept. We also show that such an equilibrium exists. In Section 4, the main result is established: our non-cooperative support for the ANBS. Section 5 concludes.

\subsection{The Bargaining Game}

We consider the bargaining game $\Gamma(N, M, V)$. The set of players is denoted by $N$, and its members are indexed from 1 until $n$. The game is played for potentially infinitely many periods $t=0,1,2, \ldots$. In each period, one player acts as the proposer. In period $t=0$, the proposer is determined in an arbitrary way. In all later periods, the proposer is chosen by a Markov chain. The probability distribution on the players in period $t>0$ depends on the identity of the proposer in period $t-1$. The entry $m_{i j}$ of the matrix $M$ is the probability that player $j$ will propose in period $t$ given that player $i$ has proposed in period $t-1$. All entries of $M$ are nonnegative and for each $i \in N$, it is true that $\sum_{j=1}^{n} m_{i j}=1$. The set $V$ corresponds to all feasible payoffs. We denote $V \cap \mathbb{R}_{+}^{n}$ by $V_{+}$. Our assumptions are as follows.

(A1) The set $V$ is closed, convex, and comprehensive from below. The origin lies in the interior of $V$. The set $V_{+}$is bounded and all weakly Pareto-efficient points in $V_{+}$are also strongly Pareto-efficient.

(A2) The matrix $M$ is irreducible.

We denote the interior and boundary of a set $X$ by $\operatorname{int}(X)$ and $\partial X$ respectively. A vector $\eta$ with $\|\eta\|=1$ is said to be normal to the convex set $V$ at a point $\bar{v} \in V$ if $(v-\bar{v})^{\top} \eta \leq 0$ for every $v \in V$. The set of all vectors $\eta$ normal to $V$ at $\bar{v}$ is called the normal to $V$ at $\bar{v}$.

(A3) There is a unique vector in the normal to $V$ at every $v \in \partial V \cap \mathbb{R}_{+}^{n}$. 
The assumption that all weakly Pareto-efficient points in $V_{+}$are also strongly Pareto-efficient is essential to our results. As we show later in Section 3, this assumption implies that a proposal of a player $i$ gives all other players their respective reservation payoffs. Thus a proposer always extracts the full surplus from all other players. Our assumptions with respect to $V$ are similar to those in Merlo and Wilson (1995) and Miyakawa (2006).

The game proceeds as follows. In any period $t$, first the proposer is chosen in the aforementioned way. Next, the proposer offers a vector $v \in V$. Then, all players (including the proposer) decide sequentially whether to accept or reject the offer $v$, where for the sake of simplicity we assume that player $i$ responds before player $i+1$. We define the set $S(i)$ consisting of player $i$ and all its successors by $S(i)=\{j \in N \mid j \geq i\}$. If all players have accepted the vector $v$ in period $t$, the game ends and each player $i$ receives a payoff of $v_{i}$. As soon as one player rejects $v$, period $t+1$ starts with probability $\delta$, and the game ends with probability $1-\delta$. In the latter case, as well as in the case of perpetual disagreement, all players receive zero payoff. We assume that players maximize expected payoffs.

We denote by $H_{i}^{\mathrm{p}}$ the set of histories after which player $i$ has to make a proposal and by $H_{i}^{\mathrm{r}}$ the set of histories after which player $i$ has to respond to a proposal. Then, a strategy for player $i$ is a map $s_{i}: H_{i}^{\mathrm{p}} \cup H_{i}^{\mathrm{r}} \rightarrow V \cup\left\{\right.$ Yes, No\}, where $s_{i}\left(H_{i}^{p}\right) \subset V$ and $s_{i}\left(H_{i}^{r}\right) \subset\{$ Yes, No .

Player $i$ 's strategy is stationary if the same proposal is made at all histories $H_{i}^{\mathrm{p}}$ and if the action taken at any history $H_{i}^{\mathrm{r}}$ depends only on the current proposal and the current proposer. ${ }^{2}$

A Nash equilibrium is a profile of strategies from which no player has an incentive to unilaterally deviate. A subgame perfect Nash equilibrium (SPE) is a profile of strategies such that its restriction to any subgame is a Nash equilibrium of that subgame.

A stationary subgame perfect Nash equilibrium (SSPE) is a profile of stationary strategies which is an SPE.

\subsection{Analysis of Stationary Equilibrium}

In this section, we characterize the set of subgame perfect equilibria in stationary strategies. Theorem 2.3.11 gives the necessary and sufficient conditions for a strategy profile to be an SSPE and Theorem 2.3.12 asserts that an SSPE exists.

The analysis in this section resembles Kalandrakis (2004), but some important differences should be noted: We conclude rather than assume that agreement is immediate in SSPE and we do not impose assumptions on the behavior of players who are indifferent between acceptance and rejection of some proposal. Furthermore, Banks and Duggan (2000) have given an equilibrium analysis similar to the one developed in this section, but not covering the general proposer protocol under consideration here.

Consider a profile of stationary strategies. It can be described by an $n \times n$-matrix $\Theta$, where

\footnotetext{
${ }^{2}$ This notion of stationarity is weaker than the notion of subgame consistency due to Harsanyi and Selten [?], which implies that a player chooses the same action at any two nodes for which the continuation game is the same. For instance, suppose that rows $i$ and $j$ of $M$ are identical. Then, the continuation games after rejection of player $i$ 's proposal and that after rejection of player $j$ 's proposal are identical. Yet, our definition allows $A_{k}^{i}$ to be different from $A_{k}^{j}$ for one or more players $k \in N$.
} 
the entry $\theta_{j}^{i}$ is the payoff proposed to player $j$ by player $i$, and a collection $\mathcal{A}$ of $n^{2}$ acceptance sets, where the acceptance set $A_{j}^{i}$ is the set of vectors in $V$ which player $j$ will accept when proposed by player $i$. The set of vectors in $V$ accepted by player $j$ and his successors, if proposed by $i$, is $A_{S(j)}^{i}=\bigcap_{k \in S(j)} A_{k}^{i}$. We refer to $A^{i}=A_{S(1)}^{i}=\bigcap_{j \in N} A_{j}^{i}$ as the social acceptance set for proposer $i$.

Suppose that in period $t$, the proposal of player $i$ is rejected. With probability $1-\delta$ the game ends and all players receive zero payoff, and with probability $\delta$ period $t+1$ is reached and play proceeds according to the profile $(\Theta, \mathcal{A})$ of stationary strategies. The expected payoff to player $j$ after rejection is $r_{j}^{i}(\Theta, \mathcal{A})$. Omitting the argument $(\Theta, \mathcal{A})$ from the notation wherever possible, we refer to $r_{j}^{i}$ as the reservation payoff of player $j$ when $i$ proposes.

Proposition 2.3.1 The reservation payoff $r^{i}$ belongs to int $(V)$.

Proof: Conditional on the next period being reached, the payoffs are determined by a probability distribution on $V$ (notice that also $0 \in V$ ), so expected payoffs belong to $V$ since $V$ is convex. Since with probability $1-\delta$ the next period is not reached, these expected payoffs equal $\delta^{-1} r^{i}$, so $\delta^{-1} r^{i} \in V$. Since $0 \in \operatorname{int}(V)$, the convex combination $(1-\delta) 0+\delta \delta^{-1} r^{i}=r^{i}$ belongs to $\operatorname{int}(V)$.

One implication of Proposition 4.3.8 is that a proposer always has the option to make a proposal that strictly exceeds the reservation payoff of every player.

Proposition 2.3.2 In $S S P E$, for $j \in N$, if $v \in A_{S(j)}^{i}$, then $v_{k} \geq r_{k}^{i}$ for all $k \in S(j)$.

Proof: Suppose that $(\Theta, \mathcal{A})$ is a profile of stationary strategies such that $v \in A_{S(j)}^{i}$ but $v_{k}<r_{k}^{i}$ for some player $k \in S(j)$. Consider a history in $H_{k}^{\mathrm{r}}$, where player $k$ responds to the proposal $v$ made by player $i$. At that history player $k$ could deviate from $(\Theta, \mathcal{A})$ by rejecting $v$. In that case, an expected payoff of $r_{k}^{i}$ would result. Hence, this deviation is profitable and $(\Theta, \mathcal{A})$ cannot be an SSPE.

Proposition 2.3.2 implies that for a vector of payoffs $v$ to belong to the social acceptance set, it should satisfy $v_{j} \geq r_{j}^{i}$ for all $j \in N$.

Proposition 2.3.3 In SSPE (a) if $v \in V$ is such that $v_{n}>r_{n}^{i}$, then $v \in A_{n}^{i}$, and (b) for each $j=1, \ldots, n-1$ if $v \in A_{S(j+1)}^{i}$ is such that $v_{j}>r_{j}^{i}$, then $v \in A_{j}^{i}$.

Proof: To prove part (a) of the proposition suppose that $v \in V$ satisfies $v_{n}>r_{n}^{i}$. Consider a history where player $n$ has to respond to the proposal $v$ of player $i$. If $n$ accepts, the proposal will be implemented, so a payoff of $v_{n}$ will result, which is strictly greater than the payoff $r_{n}^{i}$ which would result from a rejection. Therefore, SSPE requires that $v \in A_{n}^{i}$.

To prove part (b) take a $j=1, \ldots, n-1$ and a $v \in A_{S(j+1)}^{i}$ such that $v_{j}>r_{j}^{i}$. Consider a history where player $j$ responds to the proposal $v$ of player $i$. If player $j$ accepts, the proposal will be implemented since all the players succeeding $j$ accept. Since rejection results in a payoff of $r_{j}^{i}$, SSPE requires that $v \in A_{j}^{i}$. 
Proposition 4.3.2 established a kind of converse of Proposition 3.2. One implication of Proposition 4.3.2 is that a vector $v \in V$ that satisfies $v_{j}>r_{j}^{i}$ for all $j \in N$ belongs to the social acceptance set $A^{i}$.

Proposition 2.3.4 In SSPE, each player's proposal $\theta^{i}$ lies in the social acceptance set $A^{i}$ for proposer $i$.

Proof: Suppose by way of contradiction that under some SSPE there is a player $i \in N$ such that $\theta^{i} \notin A^{i}$. Consider the subgame starting at a history where player $i$ is the proposer. Since $\theta^{i}$ is rejected, $r^{i}$ is the vector of expected payoffs by definition. By Proposition 4.3.8, $r^{i} \in \operatorname{int}(V)$. Consequently, there exists $v \in V$ such that $v_{j}>r_{j}^{i}$ for all $j \in N$. By the previous proposition, $v \in A^{i}$. Hence, it would be a profitable deviation for player $i$ to propose $v$ instead of $\theta^{i}$.

Proposition 2.3.5 In SSPE, $\theta_{j}^{i} \geq 0$ and $r_{j}^{i} \geq 0$ for all $(i, j) \in N \times N$.

Proof: Suppose by way of contradiction that $(\Theta, \mathcal{A})$ is an SSPE and that $\theta_{j}^{i}<0$ for some $(i, j) \in N \times N$. Consider a history where player $j$ has to respond to the proposal $\theta^{i}$. By Proposition 2.3.4, $\theta^{i} \in A^{i}$, so player $j$ will receive a strictly negative payoff if play proceeds according to $(\Theta, \mathcal{A})$. But then, it would be a profitable deviation for player $j$ to reject the proposal. Consequently, it holds that $\theta_{j}^{i} \geq 0$ for all $(i, j) \in N \times N$. It then follows that $r_{j}^{i} \geq 0$ for all $(i, j) \in N \times N$.

The next proposition shows that an equilibrium proposal of any player gives all other players their respective reservation payoffs. Thus a proposer always extracts the entire surplus from the other players.

Proposition 2.3.6 In SSPE, $\theta_{j}^{i}=r_{j}^{i}$ for all $(i, j) \in N \times N$ such that $i \neq j$.

Proof: Since $\theta^{i} \in A^{i}$ by Proposition 2.3.4, Proposition 2.3.2 implies that $\theta_{j}^{i} \geq r_{j}^{i}$ for all $j \in N$. Suppose $\theta_{k}^{i}>r_{k}^{i}$ for some $k \in N$ such that $k \neq i$. Define the vector $v$ as follows,

$$
v_{j}= \begin{cases}\theta_{i}^{i} & \text { if } j=i \\ r_{j}^{i} & \text { if } j \neq i .\end{cases}
$$

The vector $v$ is clearly non-negative and it is in $V$, because $v \leq \theta^{i}$ and $V$ is comprehensive. Furthermore, $v_{k}=r_{k}^{i}<\theta_{k}^{i}$, so the vector $v$ is dominated by $\theta^{i}$ and is therefore not strongly Pareto-efficient. Since we assume that all weakly Pareto-efficient vectors of $V_{+}$are also strongly Pareto-efficient, the vector $v$ is not weakly Pareto-efficient. Thus, there exists a vector $v^{\prime}$ such that $v_{j}^{\prime}>v_{j}$ for all $j \in N$.

We show now that $v^{\prime} \in A^{i}$. Indeed, $v_{j}^{\prime}>v_{j}=r_{j}^{i}$ for all $j \neq i$. And for player $i$ we have the inequality $v_{i}^{\prime}>v_{i}=\theta_{i}^{i} \geq r_{i}^{i}$. Thus we conclude that $v_{j}^{\prime}>r_{j}^{i}$ for all $j \in N$. Proposition 4.3.2 now implies that $v^{\prime} \in A^{i}$, as desired.

But then player $i$ has a profitable deviation at any history where he is entitled to make a proposal, namely propose the vector $v^{\prime}$ rather than $\theta^{i}$. Indeed, the vector $v^{\prime}$ is accepted and results in a payoff of $v_{i}^{\prime}>\theta_{i}^{i}$ to player $i$.

Proposition 3.7 claims that all players make a proposal belonging to the boundary of $V$. 
Proposition 2.3.7 In $S S P E, \theta^{i} \in \partial V$ for each $i \in N$.

Proof: Suppose by way of contradiction that there is a player $i \in N$ such that $\theta^{i} \in \operatorname{int}(V)$. Equivalently, there exists $v \in V$ such that $v_{j}>\theta_{j}^{i}$ for all $j \in N$. By the immediate agreement property, $\theta^{i} \in A^{i}$. This implies that $v \in A^{i}$ as well. But then it would be a profitable deviation for player $i$ to propose $v$ rather than $\theta^{i}$.

The previous propositions are collected in the following theorem.

Theorem 2.3.8 Suppose $(\Theta, \mathcal{A})$ is an SSPE profile inducing reservation payoffs $r^{i}$. For each $i \in N$

$$
\begin{aligned}
& \theta^{i} \in A^{i} \cap \partial V \cap \mathbb{R}_{+}^{n}, \\
& r^{i}=\delta \sum_{k=1}^{n} m_{i k} \theta^{k}, \\
& \theta_{j}^{i}=r_{j}^{i}, j \in N \backslash\{i\}, \\
& A_{S(j)}^{i} \subset \cap_{k \in\{j, \ldots, n\}}\left\{v \in V \mid v_{k} \geq r_{k}^{i}\right\}, j \in N, \\
& A_{n}^{i} \supset\left\{v \in V \mid v_{n}>r_{n}^{i}\right\}, \\
& A_{j}^{i} \supset\left\{v \in A_{S(j+1)}^{i} \mid v_{j}>r_{j}^{i}\right\}, j \in\{1, \ldots, n-1\} .
\end{aligned}
$$

In what follows, we establish the converse; the conditions of Theorem 2.3.8 characterize the set of SSPE. To do so, we will first state the well-known one-shot deviation principle.

Proposition 2.3.9 Let $(\Theta, \mathcal{A})$ be a stationary strategy profile satisfying (1)-(6). If there is a subgame such that some player has a profitable deviation, then there is a subgame where he has a profitable one-shot deviation.

Here, a one-shot deviation in a subgame is a single deviation by the player at the root of the subgame. The proof of this principle is standard in the literature and is based on the optimality principle from dynamic programming. We will show next that no player has a profitable one-shot deviation from a profile of strategies satisfying conditions (1)-(6).

Proposition 2.3.10 Let $(\Theta, \mathcal{A})$ be a stationary strategy profile satisfying $(1)-(6)$. There is no subgame where a player has a profitable one-shot deviation.

Proof: Consider the subgame at a history $h \in H_{i}^{\mathrm{p}}$. Suppose player $i$ has a one-shot deviation involving a proposal $v^{i}$ different from $\theta^{i}$. If $v^{i}$ does not belong to $A^{i}$, it leads to a payoff $r_{i}^{i}$ for player $i$. Since $\theta_{j}^{i}=r_{j}^{i}$ for all $j \neq i$ and $r^{i} \in \operatorname{int}(V)$, the Pareto-efficiency of $\theta^{i}$ implies $\theta_{i}^{i}>r_{i}^{i}$, so the deviation is not profitable. If $v^{i}$ belongs to $A^{i}$, then, for $j \neq i, v_{j}^{i} \geq r_{j}^{i}=\theta_{j}^{i}$. Now $v_{i}^{i} \leq \theta_{i}^{i}$, since otherwise the Pareto-efficiency of $\theta^{i}$ would be violated. The deviation is not profitable.

Consider a subgame at a history $h \in H_{j}^{\mathrm{r}}$ where player $j$ has to respond to a proposal $v$ made by player $i$. If $j \in\{1, \ldots, n-1\}$ and $v \notin A_{S(j+1)}^{i}$, then either action by player $j$ leads to a payoff of $r_{j}^{i}$ and hence the deviation is not profitable. Suppose now that either $j=n$ or $j \in\{1, \ldots, n-1\}$ and $v \in A_{S(j+1)}^{i}$. If $v \in A_{j}^{i}$ then the original strategy leads to a payoff of $v_{j}$ 
while the deviation yields a payoff of $r_{j}^{i}$. Since $v_{j} \geq r_{j}^{i}$ by condition (4), the deviation is not profitable. If $v \notin A_{j}^{i}$ then the original strategy leads to a payoff of $r_{j}^{i}$ while the deviation yields a payoff of $v_{j}$. Since $v_{j} \leq r_{j}^{i}$ by conditions (5)-(6), the deviation is not profitable.

The previous two propositions imply that the strategies conforming to the conditions of Theorem 2.3.8 are subgame perfect. Since they are also stationary, we have the following.

Theorem 2.3.11 The strategy profile $(\Theta, \mathcal{A})$ fulfills conditions $(1)-(6)$ if and only if it is an SSPE.

If a matrix $\Theta$ of proposals is part of an SSPE, then it is part of many SSPE's. This inessential multiplicity has two sources. First, if a responding player is proposed exactly the reservation payoff, then our characterization restricts behavior only if the proposal on the table is the equilibrium proposal. This is reflected by the fact that each point on the boundary of the social acceptance set (except the relevant equilibrium proposal) may or may not be an element of that set in SSPE. Second, if a proposal lies outside a social acceptance set, it is indeterminate which player will reject the proposal. Consider for example the case where $N=\{1,2,3,4\}$ and $r^{1}=(1,1,1,1)$. Now suppose that player 1 has proposed $v=(2,2,0,0)$ in some subgame. Since $v_{3}<r_{3}^{1}$ and $v_{4}<r_{4}^{1}$, Proposition 2.3.2 implies that $v \notin A^{1}$, and by Proposition 2.3.4 $v \neq \theta^{1}$. It is also true that $v \notin A_{4}^{1}$ : If the node where player 4 has to respond is reached, that player effectively chooses between a payoff of 1 and a payoff of 0 , so SSPE requires rejection of the proposal. However, the SSPE characterization leaves indeterminate whether players 1, 2, and 3 will accept or reject $v$. Consequently, there is an SSPE for any configuration of responses by these players. In particular, player 3 may accept $v$ in SSPE although $v_{3}<r_{3}^{1}$, and player 2 may reject $v$ although $v_{2}>r_{2}^{1}$. This reasoning even extends to player 1: In SSPE, it is possible that $v \notin A_{1}^{1}$ although $v_{1}>r_{1}^{1}$ and player 1 is the proposer. However, this does not mean that player 1 may reject his own proposal on the equilibrium path, since the SSPE characterization requires the specific proposal $\theta^{1}$ to be made and immediately accepted by all players.

Theorem 2.3.12 An SSPE exists.

For a proof of SSPE existence, we refer to Kalandrakis (2004).

\subsection{The Limit Equilibrium}

Our proofs so far did not rely on Assumptions A2 and A3. They will be needed for the results of this section. Since the matrix $M$ is irreducible, it has a unique stationary distribution denoted by $\mu$. Recall that the stationary distribution $\mu$ is a probability distribution on the set of players $N$ satisfying the equation $\mu M=\mu$. Furthermore, irreducibility of $M$ implies that all states occur with positive probability under the stationary distribution, that is $\mu_{i}>0$ for each $i \in N$. If the matrix $M$ was reducible, the state space of $M$ could be partitioned into several communicating classes. In this case, one obtains results analogous to those in the sequel within each communicating class. 
Theorem 2.4.2 below is the main result of the paper. As the continuation probability goes to one, along any sequence of stationary subgame perfect equilibria of $\Gamma$, the equilibrium proposal of all players converges to the same limit. This common limit is the asymmetric Nash bargaining solution weighted by the stationary distribution $\mu$, denoted $\mu$-ANBS.

Definition 2.4.1 The asymmetric Nash product with weights $\mu$ is the function $\rho: V_{+} \rightarrow \mathbb{R}$ defined by

$$
\rho(v)=\prod_{i \in N}\left(v_{i}\right)^{\mu_{i}} .
$$

The $\mu$-ANBS is the unique maximizer of the function $\rho$ on the set $V_{+}$.

Theorem 2.4.2 Let $\left\{\delta_{h}\right\}_{h \in \mathbb{N}}$ be a sequence of continuation probabilities in $[0,1)$ converging to 1. For each $h$, let $\Theta\left(\delta_{h}\right)$ be a matrix of proposals in some SSPE of the game $\Gamma$ with continuation probability $\delta_{h}$. Then the limits $\lim _{h \rightarrow \infty} \theta^{i}\left(\delta_{h}\right)$ exist for each $i \in N$. All limits are equal to the $\mu-A N B S$.

Let $\delta_{h}$ and $\Theta\left(\delta_{h}\right)$ be as in Theorem 2.4.2. The sequence $\left\{\Theta\left(\delta_{h}\right)\right\}$ has a convergent subsequence, as it lies in the compact set $V_{+}^{n}$, the Cartesian product of $n$ copies of $V_{+}$. For the remainder of this section, we will fix any such convergent subsequence and denote its limit by $\bar{\Theta}$. Since the convergent subsequence considered is arbitrary, to prove Theorem 2.4 .2 it is sufficient to show that each column of the matrix $\bar{\Theta}$ is the $\mu$-ANBS.

We now give a brief overview of the argument. First we show that along the sequence $\left\{\Theta\left(\delta_{h}\right)\right\}$ of equilibria the proposals of all players converge to a common limit, say the point $\bar{\theta} \in V$. We then compute the tangent space to the set $\partial V$ at the point $\bar{\theta}$ by considering the pairwise differences of the equilibrium proposals of players $i$ and $n$, and show they converge to zero at the same speed as $1-\delta_{h}$. In fact, we are able to compute the limits of the vectors $\left(\theta^{i}\left(\delta_{h}\right)-\theta^{n}\left(\delta_{h}\right)\right) /\left(1-\delta_{h}\right)$ explicitly, which are then shown to span the tangent space to the set $\partial V$ at the point $\bar{\theta}$. Using this result we show next that the tangent space at $\bar{\theta}$ is orthogonal to the gradient of the asymmetric Nash product with weights $\mu$, thereby showing that $\bar{\theta}$ is the $\mu$-ANBS.

Proposition 2.4.3 All columns of $\bar{\Theta}$ are identical.

Proof: For any $i \neq j$, it follows from the SSPE characterization that

$$
\bar{\theta}_{j}^{i}=\sum_{k=1}^{n} m_{i k} \bar{\theta}_{j}^{k} .
$$

Suppose that, contrary to the proposition, not all limit proposals $\bar{\theta}^{1}, \ldots, \bar{\theta}^{n}$ are the same. Let $j \in N$ be such that in the limit not all players propose the same to player $j$, and choose $\bar{\theta}_{j}^{\diamond}$ to be either $\min _{i \in N}\left\{\bar{\theta}_{j}^{i}\right\}$ or $\max _{i \in N}\left\{\bar{\theta}_{j}^{i}\right\}$, whichever is not equal to $\bar{\theta}_{j}^{j}$. Define $N^{\diamond}=\left\{i \in N \mid \bar{\theta}_{j}^{i}=\bar{\theta}_{j}^{\diamond}\right\}$. For any $i \in N^{\diamond}$, we have

$$
\bar{\theta}_{j}^{\diamond} \bar{\theta}_{j}^{\diamond} \sum_{k \in N^{\diamond}} m_{i k}+\sum_{k \in N \backslash N^{\diamond}} m_{i k} \bar{\theta}_{j}^{k}
$$


which is equivalent to

$$
\bar{\theta}_{j}^{\diamond} \sum_{k \in N \backslash N^{\diamond}} m_{i k}=\sum_{k \in N \backslash N^{\diamond}} m_{i k} \bar{\theta}_{j}^{k} .
$$

Suppose first that for some $i \in N^{\diamond}$,

$$
\sum_{k \in N \backslash N^{\diamond}} m_{i k}>0
$$

Then

$$
\bar{\theta}_{j}^{\diamond}=\frac{\sum_{k \in N \backslash N^{\diamond}} m_{i k} \bar{\theta}_{j}^{k}}{\sum_{k \in N \backslash N^{\diamond}} m_{i k}},
$$

which contradicts the fact that either $\bar{\theta}_{j}^{\diamond}<\bar{\theta}_{j}^{k}$ for all $k \in N \backslash N^{\diamond}$ or $\bar{\theta}_{j}^{\diamond}>\bar{\theta}_{j}^{k}$ for all $k \in N \backslash N^{\diamond}$. Therefore, $m_{i k}=0$ for all $(i, k) \in N^{\diamond} \times\left(N \backslash N^{\diamond}\right)$ and thus $N^{\diamond}$ is an absorbing set. Since $N^{\diamond} \neq N$, this contradicts the irreducibility assumption on $M$.

We denote a column of $\bar{\Theta}$ by $\bar{\theta}$. For $i \in N \backslash\{n\}$, we define

$$
d^{i}\left(\delta_{h}\right)=\frac{1}{1-\delta_{h}}\left(\theta^{i}\left(\delta_{h}\right)-\theta^{n}\left(\delta_{h}\right)\right)
$$

Let $D\left(\delta_{h}\right)$ be the $n \times(n-1)$-matrix with columns $d^{1}\left(\delta_{h}\right), \ldots, d^{n-1}\left(\delta_{h}\right)$.

The rest of the proof is organized as follows. In Proposition 2.4.5 we compute the limits of $d^{i}\left(\delta_{h}\right)$ as $h$ goes to infinity. Proposition 2.4.6 establishes that the limit of $\left\{d^{i}\left(\delta_{h}\right)\right\}$ belongs to the tangent space to $\partial V$ at $\bar{\theta}$. We then proceed to show in Proposition 2.4.8 that the limits of $d^{1}\left(\delta_{h}\right), \ldots, d^{n-1}\left(\delta_{h}\right)$ are linearly independent and thus span the tangent space to $\partial V$ at $\bar{\theta}$. And finally, Proposition 2.4.9 establishes that the gradient of the Nash product with weights $\mu$ at the point $\bar{\theta}$ is orthogonal to the tangent space of $V$, thereby showing that $\bar{\theta}$ is the $\mu$-ANBS.

For a matrix $A$, we denote by $A_{-i}$ and $A^{-i}$ the matrix $A$ without its $i^{\text {th }}$ row and column, respectively. We write $\mathbf{1}$ for a column vector of ones and $I$ for the identity matrix.

For $j \in N$, we define the matrix $L(j)$ by

$$
L(j)=\left[M^{\top}-I\right]_{-n}^{-j}
$$

Thus $L(j)$ is the $(n-1) \times(n-1)$-matrix obtained from $M^{\top}-I$ by deleting column $j$ and row $n$. Proposition 2.4.4 is an auxiliary result used in the proof of Proposition 2.4.5.

Proposition 2.4.4 The matrix $L(j)$ is invertible for all $j \in N$.

Proof: Suppose $L(j)$ is singular. Let $a$ be a non-zero vector such that $\left[M^{\top}-I\right]_{-n}^{-j} a=0$. Since the elements in any column of the matrix $M^{\top}-I$ add up to zero, we also have the equation $\left(M^{\top}-I\right)_{n}^{-j} a=0$, so $\left[M^{\top}-I\right]^{-j} a=0$. By using $\left[M^{\top}-I\right]^{-j}=\left(M^{\top}-I\right) I^{-j}$ and defining $b=I^{-j} a$, we see that $\left(M^{\top}-I\right) b=0$. Thus the vector $b$ is an eigenvector of $M^{\top}$ associated with eigenvalue 1 . By the Perron-Frobenius theorem, any non-zero eigenvector of $M^{\top}$ associated with eigenvalue 1 is a strictly positive vector. However, since $b_{j}=0$, we have obtained a contradiction. Consequently, the matrix $L(j)$ is invertible. 
Proposition 2.4.5 The sequence $\left\{D\left(\delta_{h}\right)\right\}$ of matrices converges to the matrix $\bar{D}$ with rows given by $\bar{d}_{j}=\bar{\theta}_{j} \mathbf{1}^{\top} L^{-1}(j)$ for $j \in N$.

Proof: We fix $h$ and denote $\delta_{h}$ by $\delta, \theta^{i}\left(\delta_{h}\right)$ by $\theta^{i}$, and $d^{i}\left(\theta^{h}\right)$ by $d^{i}$.

For each $j \in N$ and $i \in N \backslash\{j, n\}$,

$$
\begin{aligned}
d_{j}^{i}(1-\delta) & =\theta_{j}^{i}-\theta_{j}^{n}=\delta \sum_{k=1}^{n} m_{i k} \theta_{j}^{k}-\theta_{j}^{n} \\
& =\delta \sum_{k=1}^{n} m_{i k}\left(\theta_{j}^{k}-\theta_{j}^{n}\right)+\delta \theta_{j}^{n}-\theta_{j}^{n},
\end{aligned}
$$

where we use conditions (1) and (5) for the second equality, so

$$
d_{j}^{i}=\delta \sum_{k=1}^{n-1} m_{i k} d_{j}^{k}-\theta_{j}^{n}
$$

We have found that

$$
\theta_{j}^{n}=\delta \sum_{k \notin\{i, n\}} m_{i k} d_{j}^{k}+\left(\delta m_{i i}-1\right) d_{j}^{i}, \quad j \in N, i \in N \backslash\{j, n\} .
$$

Similarly, for $j \neq n$,

$$
\begin{aligned}
d_{j}^{j}(1-\delta) & =\theta_{j}^{j}-\delta \sum_{k=1}^{n} m_{n k} \theta_{j}^{k} \\
& =\theta_{j}^{j}-\delta \sum_{k=1}^{n} m_{n k}\left(\theta_{j}^{k}-\theta_{j}^{n}\right)-\delta \theta_{j}^{n} \\
& =\theta_{j}^{j}-\theta_{j}^{n}-\delta \sum_{k=1}^{n} m_{n k}\left(\theta_{j}^{k}-\theta_{j}^{n}\right)+(1-\delta) \theta_{j}^{n}
\end{aligned}
$$

where we use conditions (1) and (5) for the first equality, so

$$
d_{j}^{j}=d_{j}^{j}-\delta \sum_{k=1}^{n-1} m_{n k} d_{j}^{k}+\theta_{j}^{n}
$$

We have found that

$$
\theta_{j}^{n}=\delta \sum_{k=1}^{n-1} m_{n k} d_{j}^{k}, \quad j \in N \backslash\{n\} .
$$

We write (7)-(8) in vector-matrix notation as

$$
\theta_{j}^{n} \mathbf{1}^{\top}=d_{j}\left(\delta M^{\top}-I\right)_{-n}^{-j}, \quad j \in N
$$

The matrix $\left(M^{\top}-I\right)_{-n}^{-j}$ is invertible by Proposition 2.4.4, and so is the matrix $\left(\delta M^{\top}-I\right)_{-n}^{-j}$ for $\delta$ close enough to one. Thus, for every $j \in N$, we can solve the above system for $d_{j}$ as

$$
d_{j}=\theta_{j}^{n} \mathbf{1}^{\top}\left[\left(\delta M^{\top}-I\right)_{-n}^{-j}\right]^{-1} .
$$


As $\delta_{h}$ goes to one, the sequence $\theta_{j}^{n}\left(\delta_{h}\right)$ converges to $\bar{\theta}_{j}$ by Proposition 2.4.3. Thus the sequence $d_{j}\left(\delta_{h}\right)$ converges to $\bar{\theta}_{j} \mathbf{1}^{\top} L^{-1}(j)$, as desired.

Proposition 2.4.5 expresses each row $j$ of the matrix $\bar{D}$ as the sum of the rows of the matrix $L^{-1}(j)$ multiplied by the scalar $\bar{\theta}_{j}$.

We show now that each column of the matrix $\bar{D}$ is orthogonal to the normal vector of $V$ at the point $\bar{\theta}$, which is unique by Assumption A3. This is equivalent to saying that each column of the matrix $\bar{D}$ belongs to the tangent space of $\partial V$ at $\bar{\theta}$. We let $\operatorname{span}(\bar{D})$ denote the column span of the matrix $\bar{D}$.

Proposition 2.4.6 It holds that $\operatorname{span}(\bar{D})$ is orthogonal to the normal vector of $V$ at $\bar{\theta}$.

Proof: Let $\eta^{i}\left(\delta_{h}\right)$ denote the normal vector of $V$ at the point $\theta^{i}\left(\delta_{h}\right)$. Since $\left\{\theta^{i}\left(\delta_{h}\right)\right\}$ converges to $\bar{\theta}$, the sequence $\left\{\eta^{i}\left(\delta_{h}\right)\right\}$ converges to $\bar{\eta}$, the normal vector to the set $V$ at the point $\bar{\theta}$. By the definition of the normal vector,

$$
\eta^{n}\left(\delta_{h}\right)^{\top}\left(\theta^{i}\left(\delta_{h}\right)-\theta^{n}\left(\delta_{h}\right)\right) \leq 0 \text { and } \eta^{i}\left(\delta_{h}\right)^{\top}\left(\theta^{i}\left(\delta_{h}\right)-\theta^{n}\left(\delta_{h}\right)\right) \geq 0
$$

Dividing by $1-\delta_{h}$ and passing to the limit yields the inequalities $\bar{\eta}^{\top} \bar{d}^{i} \leq 0$ and $\bar{\eta}^{\top} \bar{d}^{i} \geq 0$, therefore $\bar{\eta}^{\top} \bar{d}^{i}=0$, as desired.

Propositions 2.4.7 and 2.4.8 address the dimension of $\operatorname{span}(\bar{D})$. We show that the columns of $\bar{D}$ are linearly independent, thus establishing that $\operatorname{span}(\bar{D})$ equals the tangent space of $\partial V$ at $\bar{\theta}$.

For $j \in N$, let $K_{j}$ be the sum of the rows of the matrix $L^{-1}(j)$, thus

$$
K_{j}=\mathbf{1}^{\top} L^{-1}(j)
$$

Define $K$ as the $n \times(n-1)$-matrix with rows $K_{j}$. Proposition 2.4.7 expresses all rows of $K$ in terms of rows of $L^{-1}(n)$ and the stationary distribution $\mu$ induced by $M$.

Proposition 2.4.7 Any combination of $n-1$ distinct rows of the matrix $K$ is linearly independent. Furthermore,

$$
K_{j}=\mathbf{1}^{\top} L^{-1}(j)=\mathbf{1}^{\top} L^{-1}(n)-\frac{1}{\mu_{j}}\left(L^{-1}(n)\right)_{j}, \quad j \in N \backslash\{n\} .
$$

Proof: We define $x=\left[M^{\top}-I\right]_{-n}^{n}$. Consider some $j \in N \backslash\{n\}$. It can be verified by a direct 
computation that

$$
L^{-1}(j)=\left[\begin{array}{c}
\left(L^{-1}(n)\right)_{1}-\frac{\left(L^{-1}(n) x\right)_{1}}{\left(L^{-1}(n) x\right)_{j}}\left(L^{-1}(n)\right)_{j} \\
\vdots \\
\left(L^{-1}(n)\right)_{j-1}-\frac{\left(L^{-1}(n) x\right)_{j-1}}{\left(L^{-1}(n) x\right)_{j}}\left(L^{-1}(n)\right)_{j} \\
\left(L^{-1}(n)\right)_{j+1}-\frac{\left(L^{-1}(n) x\right)_{j+1}}{\left(L^{-1}(n) x\right)_{j}}\left(L^{-1}(n)\right)_{j} \\
\vdots \\
\left(L^{-1}(n)\right)_{n-1}-\frac{\left(L^{-1}(n) x\right)_{n-1}}{\left(L^{-1}(n) x\right)_{j}}\left(L^{-1}(n)\right)_{j} \\
\frac{1}{\left(L^{-1}(n) x\right)_{j}}\left(L^{-1}(n)\right)_{j}
\end{array}\right] .
$$

The formula above is well-known in linear programming and is used to compute the simplex tableau following from a change in basis variables. By definition of the stationary distribution we have

$$
L(n) \mu_{-n}^{\top}+x \mu_{n}=0
$$

We multiply this expression by $L^{-1}(n)$ and rearrange to obtain

$$
L^{-1}(n) x=-\frac{1}{\mu_{n}} \mu_{-n}^{\top}
$$

By substitution, we find that

$$
L^{-1}(j)=\left[\begin{array}{c}
\left(L^{-1}(n)\right)_{1}-\frac{\mu_{1}}{\mu_{j}}\left(L^{-1}(n)\right)_{j} \\
\vdots \\
\left(L^{-1}(n)\right)_{j-1}-\frac{\mu_{j-1}}{\mu_{j}}\left(L^{-1}(n)\right)_{j} \\
\left(L^{-1}(n)\right)_{j+1}-\frac{\mu_{j+1}}{\mu_{j}}\left(L^{-1}(n)\right)_{j} \\
\vdots \\
\left(L^{-1}(n)\right)_{n-1}-\frac{\mu_{n-1}}{\mu_{j}}\left(L^{-1}(n)\right)_{j} \\
-\frac{\mu_{n}}{\mu_{j}}\left(L^{-1}(n)\right)_{j}
\end{array}\right] .
$$

Summing up the rows of $L^{-1}(j)$ we get

$$
\left.\mathbf{1}^{\top} L^{-1}(j)=\sum_{i \in N \backslash\{j, n\}}\left(L^{-1}(n)\right)_{i}+\frac{\mu_{j}-1}{\mu_{j}}\right)\left(L^{-1}(n)\right)_{j}=\mathbf{1}^{\top} L^{-1}(n)-\frac{1}{\mu_{j}}\left(L^{-1}(n)\right)_{j} .
$$

Therefore,

$$
K_{-n}=\left[11^{\top}-C\right] L^{-1}(n),
$$

where $C$ is the $(n-1)$-diagonal matrix with element $1 / \mu_{i}$ in column $i$.

The matrix $\left[\mathbf{1 1}^{\top}-C\right]$ is non-singular. Suppose not, then there is $y \neq 0$ such that $\left[\mathbf{1 1}^{\top}-\right.$ $C] y=0$. It follows that $\mathbf{1 1}^{\top} y=C y=\left(y_{1} / \mu_{1}, \ldots, y_{n-1} / \mu_{n-1}\right)^{\top}$, from which it follows in 
particular that $\mathbf{1}^{\top} y \neq 0$. By pre-multiplying the last equality with the row vector $\left(\mu_{1}, \ldots, \mu_{n-1}\right)$, we find that $\left(1-\mu_{n}\right) \mathbf{1}^{\top} y=\mathbf{1}^{\top} y$, a contradiction since $\mu_{n}>0$. Consequently, the matrix $\left[\mathbf{1 1}^{\top}-C\right]$ is non-singular.

It follows that $K_{-n}$ is non-singular. Since the labeling of players is arbitrary, we have shown that any combination of $n-1$ distinct rows of the matrix $K$ is linearly independent.

Proposition 2.4.8 It holds that $\bar{\theta}_{i}>0$ for all $i \in N$. The column span of the matrix $\bar{D}$ has dimension $n-1$.

Proof: We know that $\bar{\theta}_{i} \geq 0$ for each $i \in N$. Partition $N$ into the set $N_{0}$ of players $i$ such that $\bar{\theta}_{i}=0$ and the set $N_{+}$of players $i$ such that $\bar{\theta}_{i}>0$.

Suppose that the set $N_{0}$ is non-empty, so that the set $N_{+}$consists of at most $n-1$ elements. We show first that $\bar{\theta} \in \operatorname{span}(\bar{D})$ by constructing a vector $z \in \mathbb{R}^{n-1}$ such that $\bar{D} z=\bar{\theta}$. Since the rows $K_{i}$ of the matrix $K$ corresponding to the elements $i$ of the set $N_{+}$are linearly independent by Proposition 2.4.7, there exists a vector $z \in \mathbb{R}^{n-1}$ such that $K_{i} z=1$ for all $i \in N_{+}$. Then $\bar{d}_{i} z=\bar{\theta}_{i} K_{i} z=\bar{\theta}_{i}$ for all $i \in N_{+}$. Trivially, $z$ also satisfies the equations $\bar{d}_{i} z=\bar{\theta}_{i} K_{i} z=0=\bar{\theta}_{i}$ for each $i \in N_{0}$.

Let $\bar{\eta}$ be the normal vector to $V$ at the point $\bar{\theta}$. Since $\bar{\eta}$ is orthogonal to $\operatorname{span}(\bar{D})$ by Proposition 2.4.6, we have $\bar{\eta}^{\top} \bar{\theta}=0$. Since zero is in the interior of $V$ by assumption, the vector $\varepsilon \eta$ is in the set $V$ for $\varepsilon>0$ small enough. But then we have the inequality $\bar{\eta}^{\top}(\varepsilon \bar{\eta}-\bar{\theta})=$ $\varepsilon\left(\bar{\eta}^{\top} \bar{\eta}\right)>0$, contradicting the definition of a normal vector. Consequently, the set $N_{0}$ is empty. We have shown the first part of the proposition.

To prove the second part of the proposition, notice that $\bar{D}$ can be written as the product $T K$, where $T$ is a diagonal matrix with $\bar{\theta}_{i}$ in column $i$. Since $\bar{\theta}_{i}>0$ for each $i \in N$, the matrix $T$ has full rank $n$, and the matrix $K$ has rank $n-1$ by Proposition 2.4.7. This establishes the second part of the proposition.

We now proof that the gradient of the logarithm of the asymmetric Nash product with weights $\mu$ is orthogonal to the column span of the matrix $\bar{D}$. We observe that for $v \gg 0$,

$$
\ln \rho(v)=\sum_{i \in N} \mu_{i} \ln \left(v_{i}\right)
$$

Proposition 2.4.9 It holds that $\operatorname{span}(\bar{D})$ is orthogonal to the gradient of the function $\ln \rho$ at the point $\bar{\theta}$.

Proof: The gradient of $\ln \rho$ at $\bar{\theta}$ is the vector $g$ given by $g_{j}=\mu_{j} / \bar{\theta}_{j}, j \in N$. We have the following chain of equations

$$
\begin{aligned}
\sum_{j \in N} g_{j} \bar{d}_{j} & =\sum_{j \in N} \mu_{j} \mathbf{1}^{\top} L^{-1}(j) \\
& =\sum_{j \in N \backslash\{n\}} \mu_{j}\left[\mathbf{1}^{\top} L^{-1}(n)-\frac{1}{\mu_{j}}\left(L^{-1}(n)\right)_{j}\right]+\mu_{n} \mathbf{1}^{\top} L^{-1}(n) \\
& =\mathbf{1}^{\top} L^{-1}(n)-\sum_{j \in N \backslash\{n\}}\left(L^{-1}(n)\right)_{j} \\
& =\mathbf{1}^{\top} L^{-1}(n)-\mathbf{1}^{\top} L^{-1}(n)=0,
\end{aligned}
$$


where the first equality uses the result of Proposition 2.4.5, and the second one Proposition 2.4.7. This establishes the proposition.

The proof of Theorem 2.4.2 is now immediate. The column span of the matrix $\bar{D}$ is orthogonal to the normal vector of $V$ at $\bar{\theta}$ by Proposition 2.4.6, and at the same time it is orthogonal to the gradient of $\ln \rho$ at $\bar{\theta}$ by Proposition 2.4.9. Since $\operatorname{span}(\bar{D})$ has dimension $n-1$ (Proposition 2.4.8), it follows at once that the gradient of the function $\ln \rho$ is proportional to the normal vector to $V$ at $\bar{\theta}$. Hence, the point $\bar{\theta}$ is the maximizer of the function $\ln \rho$ on the strictly positive vectors in the set $V$, as well as the maximizer of the function $\rho$ on the set $V_{+}$.

\subsection{Conclusion}

In this paper we have provided further non-cooperative support to the asymmetric Nash bargaining solution. We demonstrate that existing results are instances of a much more generally valid principle. We consider a bargaining process involving any number of players, an arbitrary irreducible Markov process that determines the selection of the proposer, and any set of feasible payoffs that is bounded, convex, and has a smooth boundary. As long as no agreement is reached, negotiations break down with some fixed probability.

We fully characterize the set of subgame perfect equilibria in stationary strategies. We show that at least one such equilibrium exists and argue that in general there are many such equilibria. We continue by studying the limit of an arbitrary sequence of equilibria when the probability of breakdown goes to zero. We establish that in the limit all players make the same proposal. Moreover, this proposal is the same as the one corresponding to the asymmetric Nash bargaining solution, where the weights in the Nash product are equal to the stationary distribution of the Markov process that determines the selection of the proposer.

One implication is that if players are selected as proposer in some fixed order, then the symmetric Nash bargaining solution is achieved in the limit. This can be seen as a generalization of alternating offer bargaining to more than two players. Another implication is that if players are selected according to time-invariant probabilities, these probabilities are equal to the weights in the Nash product. The symmetric Nash bargaining solution would again result if the timeinvariant probabilities are uniform.

It is noteworthy that the bargaining power of the players is only affected by the stationary distribution of the proposer selection process. The particular shape of the set of feasible payoffs is irrelevant for the weights of the players in the Nash product, as are the particular probabilities by which the proposer in the next period is chosen conditional on the current proposer. 


\section{Optimal value commitment in bilateral bargaining}

\subsection{Introduction}

Two players bargain on how to divide a pie of unit size among themselves. They can only consume the pie once they have agreed on its division. Players are impatient and thus discount future consumption. ${ }^{3}$

We are interested in the ability to commit as a source of bargaining power. We study this question using a notion of commitment with the following two characteristics:

First, the commitment is not expressed as a share of the pie but rather in terms of the pie's time value discounted back to the beginning of the bargaining process. The simple rationale behind this specification is that a commitment should be stated in the terms which the impatient player cares about. Such "value-committing" has been introduced to the literature earlier by Li (2007) and stands in contrast to the idea of "share-committing", which is more standard in the literature.

Second, we will assume that the commitment to a certain time value is perfectly binding as long as the pie has at least the committed value. However, as soon as so much time has elapsed that even the receipt of the entire pie would not lead to the committed value anymore, the commitment is assumed to become void. To the best of our knowledge, this notion of commitment is new to the bargaining literature.

Our assumption simply means that we do not allow a player to remain committed to something which is not feasible (anymore). This form of commitment confronts the player with the following dilemma: A high commitment becomes void soon, whereas a low commitment stays in effect for a long time.

It has long been recognized that an irrevocable (and perfectly credible) commitment would be an extremely powerful tool. In fact, if only one player can make such a commitment, the strategic situation resembles that in an ultimatum game, and the committed player captures the entire surplus - a result which seems unattractively lopsided. The literature has looked for ways to obtain more attractive or reasonable results by limiting the commitment's credibility. The standard approach which has been taken is to introduce a cost at which a commitment can be revoked. For instance, Muthoo (1992) presents a model of bargaining which generalizes the Nash (1953) demand game as well as Rubinstein's (1982) well-known alternating offer bargaining procedure. The former is seen as a polar case of irrevocable commitments, and the latter as an extreme case of revocable commitments, and the cost of revoking a commitment is used as the parameter scaling between the two. A typical result in this literature is that the player with the higher cost of revoking a commitment has an advantage, see, for instance, Muthoo (1996).

A different well-established approach is that of endogenous commitments. For instance, Fershtman and Seidmann (1993) and Li (2007) consider the possibility that rejecting a proposal commits a player not to accept any worse proposal in the future, an approach which can be

\footnotetext{
${ }^{3}$ This chapter corresponds to the paper V.Britz (2010), "Optimal value commitment in bilateral bargaining," METEOR Research Memorandum RM/10/056.
} 
motivated by shifts in players' reference points. This approach has been extended and combined with the cost of revoking approach by Calabuig, Cunyat, and Olcina (2002). In Cunyat (2004), a player can choose the strength of his commitment before the bargaining starts. ${ }^{4}$

In what follows, we will study the following game: One out of two players has access to the aforementioned commitment device. That player announces his commitment level. Subsequently, a potentially infinite number of bargaining rounds follows. In each such round, one of the two players is recognized as the proposer by a draw from a time-invariant probability distribution. The proposing player makes an offer and the game ends if this offer is accepted by the opponent. In case of a rejection the next round starts. However, any consumption in the next round will be discounted by a constant factor $\delta \in(0,1)$. In line with our earlier discussion, the commitment device punishes the committed player if he accepts less than his commitment level while the pie's value is still higher than that level. But once the "moment of truth "where the pie's value shrinks below the commitment level has passed, no punishment is given. One interpretation is that the device punishes "treason" but forgives "failure". Agreeing to less than the commitment while the pie is still sufficiently valuable is akin to giving in to the opponent (treason, weakness), while making an agreement after the moment of truth is giving in to the facts after the failure of an excessively strong bargaining posture. One alternative commitment device would punish the player not only if he breaks his commitment before the moment of truth, but also as soon as the moment of truth is reached. After all, in the latter case it is clear that his promise cannot be fulfilled anymore. It can be shown that this seemingly stronger commitment device does not confer any bargaining power and is therefore not useful. We will see, however, that the more flexible commitment device which we propose, does confer substantial bargaining power.

Using a slight refinement of subgame-perfect Nash equilibrium, we find immediate and efficient agreement on a unique division of the pie. If the pie shrinks very rapidly, then the ability to commit is extremely valuable. The committed player can obtain almost the entire surplus even if his proposal power is close to zero. If the pie shrinks very slowly, commitment creates less bargaining power and the recognition probabilities become more important in determining the allocation of the surplus. In the limit as $\delta$ goes to one, proposal power and commitment power are "equally important" in the following sense: If one player can commit and the other player has a recognition probability close to one, then the surplus is shared almost equally.

We present extensions of the model to games where both players can make a commitment before the bargaining starts. If they do so sequentially, then in the limit as $\delta$ goes to one, the first mover receives a share between one half and two thirds of the pie, depending on the recognition probabilities. With irrevocable and permanent commitment, one would expect the first mover to obtain the entire surplus.

We also consider the case where players make their commitments simultaneously. With irrevocable and permanent commitment, one would expect all efficient pie divisions to be supported

\footnotetext{
${ }^{4}$ In a different stream of literature, players can be of a fully rational type or of a stubborn type. Stubbornness is then a form of commitment. A typical issue within that literature is the possible incentive of a rational player to try and mimic a stubborn type. Well-known examples are Abreu and Gul (2000) and Kambe (1999). In this paper, we do not consider different types of the same player.
} 
by equilibria irrespective of the value of the discount factor. With the notion of commitment which we suggest, this is no longer true. If the discount factor is chosen sufficiently large, then we find a rather narrow range of efficient divisions which are supported by equilibria. More precisely, the share of the pie whose allocation is left unpredicted by the equilibrium concept in the limit is at most one fifth. Moreover, a player can never receive less than one third of the pie in an equilibrium with $\delta$ close to one - even with arbitrarily small proposal power. The equal split is the unique division with the property that it can be supported by an equilibrium regardless of the parameter choices for the discount factor and the recognition probabilities.

The rest of the paper is organized as follows: In the next section, we formally describe the game in which only a single player can make a commitment. In section 3 , we study the bargaining stage of that game and solve for the equilibrium given the choice of commitment. In section 4 , the game as a whole is solved and the optimal commitment is thus derived. In section 5 , the game is extended to the case where both players can choose a commitment before the bargaining starts. Again, an analysis of the bargaining stage given the commitment levels is given. The conclusions of section 4 will be essential for this analysis. Section 6 deals with the optimal choice of commitments by both players. Section 7 concludes.

\subsection{The game with one committed player}

The player set is $N=\{1,2\}$. The two players have a perfectly divisible pie of unit size at their disposal. They consume the pie once they have agreed on its division. Each player's instantaneous utility is equal to his consumption of pie, but future consumption is discounted by a constant and common factor $\delta \in(0,1)$. This implies that at any time $t$, the players can divide among themselves a surplus of value $\delta^{t}$. In the sequel, we will mean by the surplus the time value, discounted to time $t=0$, of the pie to be divided.

The game $G$ consists of a commitment stage and a bargaining stage. The game starts with the commitment stage in which a player (without loss of generality, we suppose it is player 1 ) chooses a level of commitment $c_{1} \in[0,1]$. The ensuing bargaining stage is set in discrete time $t=0,1, \ldots$. At the start of each such round $t$, one player is recognized as the proposer according to the probability distribution $\left(\beta_{1}, \beta_{2}\right)$, where $\beta_{1}+\beta_{2}=1$ and $\beta_{k}>0$ for both $k=1,2$. This player then proposes a division of the surplus, i.e. a pair $\left(x_{1}, x_{2}\right) \in \mathbb{R}_{+}^{2}$ such that $x_{1}+x_{2} \leq \delta^{t}$. If the other player rejects the proposal, round $t+1$ starts. If the other player accepts the proposal, it is implemented and the game ends with the following payoffs for the players:

$$
\begin{aligned}
u_{1}\left(x_{1}, c_{1}, t\right) & = \begin{cases}x_{1}-\lambda & \text { if } x_{1}<c_{1} \leq \delta^{t} \\
x_{1} & \text { otherwise }\end{cases} \\
u_{2}\left(x_{2}\right) & =x_{2}
\end{aligned}
$$

If players disagree forever, their payoffs are zero.

If $c_{1} \leq \delta^{t}$, we will say that the commitment $c_{1}$ is effective at time $t$. If $c_{1}>\delta^{t}$, we say that the commitment $c_{1}$ is void at time $t$. 
The extensive form of the model admits two different interpretations. The main interpretation we use here is that a pie of size one is available but the players are impatient. Their commitments are expressed in terms of time value rather than the underlying pie itself. Another interpretation of the model is that a pie of unit size is available initially but physically shrinks by the factor $\delta$ each round, while players are indifferent to the passage of time. With this interpretation, the commitment is expressed in terms of the physical pie and expires as soon as that pie has shrunk below the commitment level.

If player 1 agrees to receive less than $c_{1}$ while the commitment is still effective, he incurs a cost $\lambda$. We are interested in commitments which are perfectly binding until they expire. Therefore, we assume that $\lambda$ is large enough so that $u_{1}\left(x_{1}, c_{1}, t\right)<0$ whenever $x_{1}<c_{1} \leq$ $\delta^{t} .{ }^{5}$ Thus, perpetual disagreement is better for player 1 than the violation of an effective commitment.

Suppose that in the game $G$, player 1 has chosen the commitment level $c_{1}$ at his initial decision node. Given $c_{1}$, the game's bargaining stage will start. We refer to this bargaining stage as the bargaining (sub-)game $G\left(c_{1}\right)$, which will be analyzed in the next section. Moreover, we will denote by $G\left(c_{1}, t\right)$ some subgame of $G\left(c_{1}\right)$ which begins with the move of nature in round $t \geq 1$ of bargaining. Given a bargaining subgame $G\left(c_{1}\right)$ and a round $t$, there are many such subgames $G\left(c_{1}, t\right)$, all of which are, however, equivalent with regard to strategies and payoffs. ${ }^{6}$

\subsection{Subgame perfect bargaining equilibrium}

In this section, we consider the bargaining subgame $G\left(c_{1}\right)$ for any given commitment level $c_{1}$, and solve for the equilibrium division of the surplus. As a solution concept, we will use a slight refinement of the well-known subgame-perfect Nash equilibrium (SPE). The purpose of the refinement is to break ties in favor of agreement. Such a behavior would follow from SPE in the entire game $G$, but has to be imposed exogenously when a bargaining subgame is considered in isolation. More formally, let $s_{i}^{B}$ be the (bargaining) strategy for player $i=1,2$ in the game $G\left(c_{1}\right)$. In accordance with the usual definition of a strategy, $s_{i}^{B}$ assigns to each history of $G\left(c_{1}\right)$ either a proposal to be made by player $i$ or a decision on whether to accept or reject the opponent's current proposal. As by the standard definition, a strategy pair $\left(\bar{s}_{i}^{B}, \bar{s}_{j}^{B}\right)$ is a subgame-perfect Nash equilibrium (SPE) if its restriction to any subgame of $G\left(c_{1}\right)$ is a Nash equilibrium in that subgame. In the following definition, we introduce a refinement of this equilibrium concept.

Definition 3.3.1 A strategy pair $\left(\bar{s}_{i}^{B}, \bar{s}_{j}^{B}\right)$ is a subgame-perfect bargaining equilibrium (SPBE) of $G\left(c_{1}\right)$ if it is an SPE and, in addition, satisfies the following conditions.

1. Suppose that under the profile $\bar{s}^{B}$, player $j=1,2$ rejects a particular proposal, say $x$, at some history, say $h$. Let $\tilde{s}_{j}^{B}$ be the strategy which accepts the proposal $x$ at $h$ but agrees

\footnotetext{
${ }^{5}$ This is ensured for any $\lambda \geq 1$. A different appealing specification is that $\lambda\left(c_{1}\right)=c_{1}$ for any $c_{1}$.

${ }^{6}$ For the analysis, it will be convenient that the random draw of the proposer in each round gives the game a stationary structure. Matters would be more complicated with a sequential-offers protocol such as Rubinstein (1982), although the flavor of the main (limit) results in this paper would be expected to carry over to such a setting as well.
} 
with strategy $\bar{s}_{j}^{B}$ at all other histories. Then, the restriction of the profile $\left(\bar{s}_{i}^{B}, \bar{s}_{j}^{B}\right)$ to the subgame starting at $h$ leads to a strictly greater payoff for player $j$ in this subgame than the restriction of the profile $\left(\bar{s}_{i}^{B}, \tilde{s}_{j}^{B}\right)$.

2. Suppose that under the profile $\bar{s}^{B}$, there is a history $h$ at which player $i=1,2$ makes a proposal, say $x$, which is subsequently rejected by player $j \neq i$. Suppose further that in the subgame following this rejection, the appropriate restriction of $\bar{s}^{B}$ leads to a payoff of $r_{i}$ for player $i$. Let $\tilde{X}$ be the set of proposals which, if made by player $i$ at history $h$, would subsequently be accepted by player $j$ under the profile $\bar{s}^{B}$. Then, it holds that $r_{i}>\tilde{x}_{i}$ for all $\tilde{x} \in \tilde{X}$.

The first condition above says that a player only rejects a proposal in SPBE when accepting the proposal would make him strictly worse off. The second condition says that a player only makes an unacceptable proposal in SPBE if this is strictly better for him than making an acceptable proposal.

In standard bargaining models without commitment, the fact that delay is costly implies that in any round there is a feasible agreement which strictly Pareto-dominates the payoff vector which would result from disagreement in that round. Under such conditions, SPE strategy profiles have the properties that agreement is reached immediately, and that a responder accepts a proposal when indifferent between acceptance and rejection. In the model at hand, however, the commitment leads to a discontinuity in the utility function. Such a discontinuity makes it possible that in some round, some feasible agreement is strictly preferred to disagreement by one player, while the other player is indifferent, and no feasible agreement makes both players strictly better off than disagreement. In such a situation, the standard SPE concept leaves it indeterminate whether or not an agreement will be reached. With SPBE as the solution concept, agreement is ensured. It can be shown that delay on the equilibrium path is inconsistent with SPE if the entire game (including the commitment stage) is considered. In this sense, the refinement from SPE to SPBE can be seen as merely technical.

We will now formalize the idea of the "moment of truth" mentioned in the introduction.

Define

$$
\tau\left(c_{1}\right)= \begin{cases}\min \left\{t \in \mathbb{N} \mid t>\ln \left(c_{1}\right) / \ln (\delta)\right\} & \text { if } c_{1} \in(0,1] \\ 0 & \text { if } c_{1}=0\end{cases}
$$

so that in round $\tau\left(c_{1}\right)$ and all later rounds, the commitment $c_{1}$ is void. We will say that the commitment $c_{1}$ expires at time $\tau\left(c_{1}\right)$.

Remark 3.3.2 The definition of $\tau\left(c_{1}\right)$ readily implies that:

1. $\delta^{\tau\left(c_{1}\right)}<c_{1}$ for all $c_{1} \in(0,1]$;

2. $\delta c_{1} \leq \delta^{\tau\left(c_{1}\right)}$ for all $c_{1} \in[0,1]$; 
3. $\tau\left(c_{1} \delta^{t}\right)=\tau\left(c_{1}\right)+t$ for all $c_{1} \in(0,1]$ and $t \in \mathbb{N}$.

These properties of $\tau\left(c_{1}\right)$ will be exploited repeatedly throughout the paper.

Consider a subgame $G\left(c_{1}, t\right)$ for any $t \geq \tau\left(c_{1}\right)$. Any such subgame is equivalent to a bargaining game without commitment. In fact, the only difference compared to the game in Rubinstein (1982) is that proposals are not made in an alternating fashion but that the proposer in each round is determined by a fixed recognition probability. The following lemma says that in such a subgame following the expiry of the commitment, the available surplus is divided in the proportion of the recognition probabilities.

Lemma 3.3.3 In any subgame $G\left(c_{1}, \tau\left(c_{1}\right)\right)$, player $k$ 's $(k=i, j)$ expected SPBE payoff is equal to $\beta_{k} \delta^{\tau\left(c_{1}\right)}$.

Proof: Binmore (1987) proves that the SPE payoffs in such a subgame are equal to $\beta_{k} \delta^{\tau\left(c_{1}\right)}$. The corresponding SPE strategy profile conforms to our definition of an SPBE strategy profile.

By setting a commitment of zero, player 1 can effectively choose to play the bargaining game without commitment, recall that $\tau(0)=0$. Lemma 3.3.3 implies that in an SPBE of that bargaining game, the surplus will be divided in the proportion of the recognition probabilities, as stated in the following corollary. As one would intuitively expect, the possibility to commit cannot weaken player 1's bargaining position compared to an analogous bargaining game without commitment.

Corollary 3.3.4 If $c_{1}=0$, then the SPBE payoffs in $G\left(c_{1}\right)$ are $\left(\beta_{1}, \beta_{2}\right)$.

We have earlier introduced $G\left(c_{1}, t\right)$ as the notation for a subgame of $G\left(c_{1}\right)$ which starts with the move of nature at round $t \geq 1$. Clearly, there are many such subgames, and each such subgame may in principle have more than one SPBE.

Definition 3.3.5 We will say that the SPBE payoffs of $G\left(c_{1}, t\right)$ are essentially unique if all SPBE of all subgames of the type $G\left(c_{1}, t\right)$ lead to the same payoffs.

In particular, Lemma 3.3.3 implies that the SPBE payoffs of $G\left(c_{1}, \tau\left(c_{1}\right)\right)$ are essentially unique for any $c_{1}$.

We now introduce the notion of a player's aspiration, which is crucial in the equilibrium analysis.

Definition 3.3.6 Suppose that for some $t \in \mathbb{N}_{0}$, the SPBE payoffs of $G\left(c_{1}, t+1\right)$ are essentially unique and equal to $\left(v_{1}^{t+1}, v_{2}^{t+1}\right)$. Then, the players' aspirations at time $t$ under the commitment $c_{1}$ are

$$
\begin{aligned}
& \left.\alpha_{1}^{t}\left(c_{1}\right)=\min \left\{x_{1} \in[0,1] \mid u_{1}\left(x_{1}, c_{1}, t\right) \geq v_{1}^{t+1}\right)\right\} \\
& \alpha_{2}^{t}\left(c_{1}\right)=v_{2}^{t+1}\left(c_{1}\right)
\end{aligned}
$$


A player's aspiration at some point in time is that amount of surplus which the player would have to receive at that point in order to realize at least his reservation utility, that is, the expected utility from delaying the agreement to the next time period. The presence of commitment in our model creates a discontinuity in the utility function of the committed player. This discontinuity leads to a distinction between what is commonly called the reservation utility and what we have here defined as the aspiration. More formally, the above definition implies the following.

$$
\begin{gathered}
\alpha_{1}^{t}\left(c_{1}\right)= \begin{cases}v_{1}^{t+1}\left(c_{1}\right) & \text { if } t \geq \tau\left(c_{1}\right) \\
\max \left\{c_{1}, v_{1}^{t+1}\left(c_{1}\right)\right\} & \text { otherwise }\end{cases} \\
\alpha_{2}^{t}\left(c_{1}\right)=v_{2}^{t+1}\left(c_{1}\right)
\end{gathered}
$$

In particular, if $\tau\left(c_{1}\right) \geq 1$, then the aspirations of players 1 and 2 at time $t=\tau\left(c_{1}\right)-1$ are $\alpha_{1}^{t}\left(c_{1}\right)=c_{1}$ and $\alpha_{2}^{t}\left(c_{1}\right)=\beta_{2} \delta^{\tau\left(c_{1}\right)}$. Intuitively, when we reason backwards from round $\tau\left(c_{1}\right)$ to round $\tau\left(c_{1}\right)-1$, then player 1's aspiration level jumps up to $c_{1}$ due to the discontinuity which occurs in the utility function. As a consequence, the sum of the aspirations before time $\tau\left(c_{1}\right)$ may exceed the available surplus so that there is no scope for an agreement.

Lemma 3.3.7 Suppose that for some $t \in \mathbb{N}_{0}$, the SPBE payoffs of $G\left(c_{1}, t+1\right)$ are essentially unique and equal to $\left(v_{1}^{t+1}, v_{2}^{t+1}\right)$. Then, the SPBE of $G\left(c_{1}, t\right)$ are essentially unique as well and are given by

$$
v_{i}^{t}\left(c_{1}\right)= \begin{cases}\alpha_{i}^{t}\left(c_{1}\right)+\beta_{i}\left(\delta^{t}-\alpha_{i}^{t}\left(c_{1}\right)-\alpha_{j}^{t}\left(c_{1}\right)\right) & \text { if } \alpha_{i}^{t}\left(c_{1}\right)+\alpha_{j}^{t}\left(c_{1}\right) \leq \delta^{t} \\ v_{i}^{t+1} & \text { otherwise }\end{cases}
$$

for $i=1,2$ and $j \neq i$.

Proof: Suppose without loss of generality that in round $t$, player $i$ is the proposer and player $j$ is the responder. Consider the case where $\alpha_{i}^{t}+\alpha_{j}^{t} \leq \delta^{t}$. (In this proof, we omit the argument $\left(c_{1}\right)$ from the notation.) We claim that in SPBE, agreement will be reached on the division $\left(\delta^{t}-\alpha_{j}^{t}, \alpha_{j}^{t}\right)$. By definition of $\alpha_{j}^{t}$ and the supposed essential uniqueness of SPBE of $G\left(c_{1}, t+1\right)$, it holds that player $j$ weakly prefers an agreement $x$ at $t$ to disagreement at $t$ if and only if $x_{j} \geq \alpha_{j}^{t}$. By the definition of SPBE, it follows that any proposal $x$ will be accepted by player $j$ at time $t$ if and only if $x_{j} \geq \alpha_{j}^{t}$. In particular, the proposal $\left(\delta^{t}-\alpha_{j}^{t}, \alpha_{j}^{t}\right)$ is acceptable to player $j$. Indeed, proposing this division is weakly preferred by player $i$ to disagreement because of the supposition that $\delta^{t}-\alpha_{j}^{t} \geq \alpha_{i}^{t}$. By the definition of SPBE, it follows that player $i$ will make an acceptable proposal. Standard arguments imply that player $i$ will not make an inefficient proposal, and not offer more than $\alpha_{j}^{t}$ to player $j$, so that the SPBE proposal is indeed exactly $\left(\delta^{t}-\alpha_{j}^{t}, \alpha_{j}^{t}\right)$. Taking into account that the proposer is chosen from the distribution $\beta$, 
we have now shown the lemma for the case where $\alpha_{i}^{t}+\alpha_{j}^{t} \leq \delta^{t}$. Suppose next that $\alpha_{i}^{t}+\alpha_{j}^{t}>\delta^{t}$. We show that no agreement is reached at $t$. As we have argued before, player $j$ weakly prefers an agreement $x$ to disagreement if and only if $x_{j} \geq \alpha_{j}^{t}$. Thus, if an agreement $x$ were reached at time $t$, then $x_{i} \leq \delta^{t}-\alpha_{j}^{t}<\alpha_{i}^{t}$. By definition of $\alpha_{i}^{t}$, the agreement $x_{i}$ gives player $i$ a strictly lower utility than he would get if there were no agreement at $t$. But player $i$ has the option to make an unacceptable proposal to player $j$, for instance $\left(\tilde{x}_{i}, \tilde{x}_{j}\right)=(1,0)$. Thus, no agreement is possible in SPBE at time $t$, so that the reservation utilities at $t$ are equal to the reservation utilities at $t+1$, as desired.

Lemma 3.3.3 and Lemma 3.3.7 provide us with an explicit backward-induction algorithm to compute the SPBE payoffs in the game $G\left(c_{1}\right)$. The former lemma says that the SPBE payoffs of $G\left(c_{1}, \tau\left(c_{1}\right)\right)$ are essentially unique, and also states these payoffs explicitly. But the latter lemma allows us to compute the essentially unique SPBE payoffs of $G\left(c_{1}, t\right)$ whenever the essentially unique SPBE payoffs of $G\left(c_{1}, t+1\right)$ are known. At each step of the backward induction procedure, Equations (9)-(10) give the aspirations in round $t$ as a function of the reservation utilities $v^{t+1}$. Then, Equation (11) translates the aspirations $\alpha^{t}$ into the reservation utilities $v^{t}$. Since the commitment $c_{1}$ expires at a finite time $\tau\left(c_{1}\right)$, we reach the payoffs $v^{0}$ after finitely many iterations and have thus computed the SPBE payoffs of the entire bargaining subgame $G\left(c_{1}\right)$. In this way, we arrive at Theorem 3.3.8, which will conclude this section. Before stating the theorem, however, we will now give some illustration of the computations carried out according to the algorithm.

Let us consider first the situation where $c_{1}+\beta_{2} \delta^{\tau\left(c_{1}\right)}>1$. (For the purpose of this illustration, we will drop the arguments $\left(c_{1}\right)$ from the notation.) Then, the backward-induction procedure begins as follows.

$$
\begin{aligned}
v^{\tau} & =\left(\beta_{1} \delta^{\tau}, \beta_{2} \delta^{\tau}\right) \\
\alpha^{\tau-1} & =\left(c_{1}, \beta_{2} \delta^{\tau}\right) \\
v^{\tau-1} & =\left(\beta_{1} \delta^{\tau}, \beta_{2} \delta^{\tau}\right)=v^{\tau}
\end{aligned}
$$

The first line follows from Lemma 3.3.3, and the second line from Definition 3.3.6. Since $c_{1}+\beta_{2} \delta^{\tau}>\delta^{\tau-1}$, Lemma 3.3.7 implies the third line. Finally, we can observe that $v^{\tau-1}=v^{\tau}$.

But now, since $c_{1}+\beta_{2} \delta^{\tau}>1 \geq \delta^{t}$ for any $t=0,1, \ldots, \tau-1$, we can iterate the argument and eventually find $v^{0}=v^{\tau}=\left(\beta_{1} \delta^{\tau}, \beta_{2} \delta^{\tau}\right)$. We depict the situation on the first of the following time-lines, where the solid line segment stands for periods where agreement is possible, and the dotted line segment stands for periods where no agreement is feasible. Player 1's commitment is so high that no agreement can be reached while the commitment is effective.

Now consider the case where $c_{1}>0$ and, furthermore, $c_{1}+\beta_{2} \delta^{\tau\left(c_{1}\right)} \leq 1$. In that case, we define

$$
a_{1}\left(c_{1}\right)=\max \left\{z \in \mathbb{N}_{0} \mid \delta^{z} \geq c_{1}+\beta_{2} \delta^{\tau\left(c_{1}\right)}\right\}
$$




$$
t=0 \quad \tau\left(c_{1}\right)
$$

In words, round $a_{1}\left(c_{1}\right)$ is the latest round of bargaining in which $c_{1}$ is effective and the surplus is sufficient to satisfy both players' aspirations. As in the previous case, the backwardinduction algorithm is initialized by the payoffs at round $\tau$ (again, we omit the arguments $\left(c_{1}\right)$ ). By the same token as before, we have

$$
\begin{aligned}
v^{\tau} & =\left(\beta_{1} \delta^{\tau}, \beta_{2} \delta^{\tau}\right) \\
& \vdots \\
v^{a_{1}+1} & =\left(\beta_{1} \delta^{\tau}, \beta_{2} \delta^{\tau}\right)=v^{\tau}
\end{aligned}
$$

But now, by definition of $a_{1}$, it holds that $c_{1}+\beta_{2} \delta^{\tau} \leq \delta^{a_{1}}$. Therefore, the second case stated in Lemma 3.3.7 applies, and we have

$$
\begin{aligned}
& v_{1}^{a_{1}}=\beta_{1}\left(\delta^{a_{1}}-\beta_{2} \delta^{\tau}\right)+\beta_{2} c_{1} \\
& v_{2}^{a_{1}}=\beta_{1} \beta_{2} \delta^{\tau}+\beta_{2}\left(\delta^{a_{1}}-c_{1}\right)
\end{aligned}
$$

Since $v_{1}^{a_{1}} \geq c_{1}$, we now have $\alpha^{a_{1}-1}=v^{a_{1}}$. In rounds $t<a_{1}$, aspirations are equal to the expected payoffs in round $t+1$, and therefore they sum up to $\delta^{t+1}<\delta^{t}$, so that agreement is always possible. Iterating this argument yields

$$
\begin{aligned}
& v_{1}^{0}\left(c_{1}\right)=\beta_{1}+\beta_{2} c_{1}-\beta_{1} \beta_{2} \delta^{\tau\left(c_{1}\right)} \\
& v_{2}^{0}\left(c_{1}\right)=\beta_{2}-\beta_{2} c_{1}+\beta_{1} \beta_{2} \delta^{\tau\left(c_{1}\right)}
\end{aligned}
$$

Again, on the second time-line a solid line indicates periods in which an agreement can be reached and dotted lines indicate periods in which delay occurs because the sum of aspirations is higher than the current surplus.

$$
t=\underset{0}{0} a_{1}\left(c_{1}\right) \quad \tau\left(c_{1}\right)
$$

The theorem below gives the SPBE payoffs in the game $G\left(c_{1}\right)$ as computed by the backward induction procedure in function of player 1's choice of $c_{1}$. 
Theorem 3.3.8 All SPBE of the bargaining subgame $G\left(c_{1}\right)$ lead to a payoff for player 1 given by the following function $\pi_{1}\left(c_{1}\right)$.

$$
\pi_{1}\left(c_{1}\right)= \begin{cases}\beta_{1}+\beta_{2} c_{1}-\beta_{1} \beta_{2} \delta^{\tau\left(c_{1}\right)} & \text { if } c_{1}+\beta_{2} \delta^{\tau\left(c_{1}\right)} \leq 1 \text { and } c_{1}>0 \\ \beta_{1} \delta^{\tau\left(c_{1}\right)} & \text { if } c_{1}+\beta_{2} \delta^{\tau\left(c_{1}\right)}>1 \\ \beta_{1} & \text { if } c_{1}=0\end{cases}
$$

In the first and third cases, an efficient agreement is reached immediately so that the payoff to player 2 equals $1-\pi_{1}\left(c_{1}\right)$. Only in the second case, delay occurs, and the payoff to player 2 is equal to $\beta_{2} \delta^{\tau\left(c_{1}\right)}$.

We emphasize that the essential uniqueness of SPBE payoffs in the bargaining subgame does not imply anything about uniqueness of SPBE strategies. For instance, in a round $t$ such that $a_{1}\left(c_{1}\right)<t<\tau\left(c_{1}\right)$, no agreement is possible, since the sum of players' aspirations exceeds the available surplus. In SPBE, no agreement can therefore occur in such a round $t$. However, it is indeterminate which proposal is made in round $t$.

\subsection{Optimal commitment}

In the previous section, we have found player 1's expected SPBE payoff in the subgame $G\left(c_{1}\right)$ for any commitment level $c_{1}$. Now we proceed to the analysis of the game $G$ and ask which level of commitment player 1 will choose in equilibrium. In the game $G$, a pair of strategies is a unilateral commitment equilibrium (UCE) if player 1's choice of commitment $\bar{c}_{1}$ satisfies $\pi_{1}\left(\bar{c}_{1}\right) \geq \pi_{1}\left(c_{1}\right)$ for all $c_{1} \in[0,1]$ and the restriction of the strategy pair to the bargaining subgame $G\left(c_{1}\right)$ is an SPBE in that subgame.

So far, we have not used the assumption that each player has a strictly positive recognition probability. It will become essential in this section.

The content of the following two lemmas is straightforward. It is shown first that in UCE, player 1 indeed makes use of the commitment device by choosing a strictly positive $c_{1}$. On the other hand, we show that in UCE, the commitment will be chosen sufficiently small so that an agreement can be reached before time $\tau\left(c_{1}\right)$.

Lemma 3.4.1 In any UCE of the game $G$, it is true that $c_{1}>0$.

Proof: Since $\lim _{c_{1} \downarrow 0}\left[c_{1}+\beta_{2} \delta^{\tau\left(c_{1}\right)}\right]=0$, we can find $c_{1}^{\prime}>0$ sufficiently small such that $c_{1}^{\prime}+\beta_{2} \delta^{\tau\left(c_{1}^{\prime}\right)} \leq 1$. Then, it follows from Theorem 3.3.8 that $\pi_{1}\left(c^{\prime}\right)=\beta_{1}+\beta_{2} c_{1}^{\prime}-\beta_{1} \beta_{2} \delta^{\tau\left(c_{1}^{\prime}\right)}$. By definition of $\tau\left(c_{1}\right)$, it holds that $c_{1}>\delta^{\tau\left(c_{1}\right)}$ for any $c_{1}>0$. If $\beta_{1}<1$, this implies that $\pi_{1}\left(c_{1}^{\prime}\right)>\beta_{1}+\beta_{2}^{2} \delta^{\tau\left(c_{1}^{\prime}\right)}$, which readily implies $\pi_{1}\left(c_{1}^{\prime}\right)>\beta_{1}$. But by Corollary 3.3.4, $\beta_{1}$ is the payoff to player 1 from the choice of $c_{1}=0$.

Lemma 3.4.2 In any UCE of the game $G$, it holds that $c_{1}+\beta_{2} \delta^{\tau\left(c_{1}\right)} \leq 1$. 
Proof: Suppose not. Then, by Theorem 3.3.8, player 1's UCE payoff in the subgame $G\left(c_{1}\right)$ equals $\beta_{1} \delta^{\tau\left(c_{1}\right)} \leq \beta_{1} \delta$. Player 1 may deviate from his choice of $c_{1}$ to a commitment level of zero. In that case, a payoff of $\beta_{1}>0$ will result. Since $\beta_{1}>\beta_{1} \delta$, this deviation is profitable, a contradiction.

The two previous lemmas show that only the first case mentioned in Theorem 3.3 .8 is relevant in a UCE of the entire game, giving rise to the following corollary.

Corollary 3.4.3 In any UCE of the game $G$, an efficient agreement is reached immediately. The commitment level $c_{1}$ of player $i$ satisfies $c_{1}>0$ and $c_{1}+\beta_{2} \delta^{\tau\left(c_{1}\right)} \leq 1$. The payoff to player 1 is given by

$$
\pi_{1}\left(c_{1}\right)=\beta_{1}+\beta_{2} c_{1}-\beta_{1} \beta_{2} \delta^{\tau\left(c_{1}\right)}
$$

and the payoff to player 2 equals $1-\pi_{1}\left(c_{1}\right)$.

The expression for player 1's payoff in the above corollary can be rewritten as $\pi_{1}\left(c_{1}\right)=$ $c_{1}+\beta_{1}\left(1-c_{1}-\beta_{2} \delta^{\tau\left(c_{1}\right)}\right)$, and the concomitant payoff of player 2 as $\beta_{2} \delta^{\tau\left(c_{1}\right)}+\beta_{2}\left(1-c_{1}-\beta_{2} \delta^{\tau\left(c_{1}\right)}\right)$. Hence, given that player 1's commitment $c_{1}$ satisfies $c_{1}>0$ and $c_{1}+\beta_{2} \delta^{\tau(c)} \leq 1$, the resulting division of the surplus can be interpreted as follows: Player 1 obtains his commitment level $c_{1}$, player 2 his resulting aspiration $\beta_{2} \delta^{\tau\left(c_{1}\right)}$, and the remainder is divided in the proportion of the recognition probabilities - as it would be if there were no commitment.

We now define a particular commitment level (depending on $\delta$ and $\beta$ ) and then show that this commitment will be chosen in UCE. Indeed, let

$$
\psi_{1}= \begin{cases}\delta^{\tilde{m}} & \text { if } \delta^{\tilde{m}} \geq \frac{1}{2-\delta \beta_{1}} \\ 1-\beta_{2} \delta^{\tilde{m}} & \text { otherwise }\end{cases}
$$

where $\tilde{m}$ is given by

$$
\tilde{m}=\min \left\{m \in \mathbb{N}_{0} \mid \delta^{m} \leq \frac{1}{1+\beta_{2} \delta}\right\}
$$

Theorem 3.4.4 In any UCE of the game $G$, player 1 commits to $\psi_{1}$. Moreover, agreement is reached immediately on the division $\left(\varphi_{1}, 1-\varphi_{1}\right)$, where $\varphi_{1}=\pi_{1}\left(\psi_{1}\right)$.

\section{Proof:}

Step 1. In UCE, agreement is reached immediately as by Corollary 3.4.3, under an effective commitment $c_{1}>0$ such that $c_{1}+\beta_{2} \delta^{\tau\left(c_{1}\right)} \leq 1$. Using the expression for the payoff in Corollary 3.4.3, the following statement is easily verified: For some $c_{1}>0$ such that $c_{1}+\beta_{2} \delta^{\tau\left(c_{1}\right)}<1$, suppose that there exists $\varepsilon>0$ sufficiently small so that $\tau\left(c_{1}+\varepsilon\right)=\tau\left(c_{1}\right)$ 
and $c_{1}+\varepsilon+\beta_{2} \delta^{\tau\left(c_{1}\right)} \leq 1$. Then, $\pi_{1}\left(c_{1}+\varepsilon\right)>\pi_{1}\left(c_{1}\right)$. Hence, $c_{1}$ cannot be the optimal choice of commitment. Conversely, we have shown that if $c_{1}$ is optimal, then either it holds that $c_{1}=\delta^{m}$ for some $m \in \mathbb{N}_{0}$, or that $c_{1}+\beta_{2} \delta^{\tau\left(c_{1}\right)}=1$.

Step 2. By construction of $\tilde{m}$, any $c_{1} \geq \delta^{\tilde{m}-1}$ would violate the condition $c_{1}+\beta_{2} \delta^{\tau\left(c_{1}\right)} \leq 1$, and can thus not be optimal by Corollary 3.4.3. We have shown that the optimal commitment level satisfies $c_{1}<\delta^{\tilde{m}-1}$.

Step 3. We show next that $c_{1} \geq \delta^{\tilde{m}}$ in UCE. To see this, suppose by way of contradiction that some $c_{1}<\delta^{\tilde{m}}$ is optimal. By definition of $\tilde{m}$, we have $c_{1}+\beta_{2} \delta^{\tau\left(c_{1}\right)}<1$. Since the inequality is strict, the argument in Step 1 above implies that $c_{1}=\delta^{m^{\prime}}$ for some $m^{\prime} \in \mathbb{N}_{0}$. But then $m^{\prime}>\tilde{m}$ because $c_{1}<\delta^{\tilde{m}}$. Plugging into the payoff function, we see that $\pi_{1}\left(\delta^{\tilde{m}}\right)>\pi_{1}\left(\delta^{m^{\prime}}\right)$. Thus, player 1 could profitably deviate from $c_{1}=\delta^{m^{\prime}}$ to a commitment of $\delta^{\tilde{m}}$, a contradiction. We have now established that $c_{1} \in\left[\delta^{\tilde{m}}, \delta^{\tilde{m}-1}\right)$ in UCE.

For clarification, we remark that $\tau\left(\delta^{\tilde{m}}\right)=\tilde{m}+1$, whereas $\tau\left(c_{1}\right)=\tilde{m}$ for any $c_{1} \in\left(\delta^{\tilde{m}}, \delta^{\tilde{m}-1}\right)$.

Step 4. In this step, we derive a condition under which there exists some $h>0$ so that $\delta^{\tilde{m}}+h+\beta_{2} \delta^{\tilde{m}} \leq 1$ and $\pi_{1}\left(\delta^{\tilde{m}}+h\right)>\pi_{1}\left(\delta^{\tilde{m}}\right)$. Rewriting the first condition, we find $h \leq 1-\left(1+\beta_{2}\right) \delta^{\tilde{m}}$. Since we are interested in $h>0$ which satisfy the first condition, we know from Corollary 3.4.3 that $\pi_{1}\left(\delta^{\tilde{m}}+h\right)=\beta_{1}+\beta_{2}\left(\delta^{\tilde{m}}+h\right)-\beta_{1} \beta_{2} \delta^{\tilde{m}}$. The condition $\pi_{1}\left(\delta^{\tilde{m}}+h\right)>$ $\pi_{1}\left(\delta^{\tilde{m}}\right)$ can then be written as $\left(\beta_{1}+\beta_{2}\left(\delta^{\tilde{m}}+h\right)-\beta_{1} \beta_{2} \delta^{\tilde{m}}\right)-\left(\beta_{1}+\beta_{2} \delta^{\tilde{m}}-\beta_{1} \beta_{2} \delta^{\tilde{m}+1}\right)>0$. Suitably rearranging the terms, this can be reduced to $h>\beta_{1} \delta^{\tilde{m}}(1-\delta)$. We are now looking for some $h$ such that $1-\left(1+\beta_{2}\right) \delta^{\tilde{m}} \geq h>\beta_{1} \delta^{\tilde{m}}(1-\delta)$. Such $h$ exists if and only if $\delta^{\tilde{m}}<\frac{1}{2-\delta \beta_{1}}$. If $\delta^{\tilde{m}} \geq \frac{1}{2-\beta_{1} \delta}$, then a commitment of $\delta^{\tilde{m}}$ is indeed optimal, as claimed in the lemma.

Step 5. Now turn to the case where $\delta^{\tilde{m}}<\frac{1}{2-\beta_{1} \delta}$. In this case, we have shown in Step 4 that there does exist $h>0$ such that $\delta^{\tilde{m}}+h+\beta_{2} \delta^{\tilde{m}} \leq 1$ and $\pi_{1}\left(\delta^{\tilde{m}}+h\right)>\pi_{1}\left(\delta^{\tilde{m}}\right)$. Thus, $\delta^{\tilde{m}}$ is not optimal, and so it follows from the conclusion of Step 3 above that the optimal $c_{1}$ belongs to the open interval $\left(\delta^{\tilde{m}}, \delta^{\tilde{m}-1}\right)$. Since this interval is open, the optimal commitment level cannot satisfy $c_{1}=\delta^{m}$ for any $m \in \mathbb{N}_{0}$. But then, by the argument in Step 1 , the optimal commitment level must satisfy $c_{1}+\beta_{2} \delta^{\tau\left(c_{1}\right)}=1$. Since $\tau\left(c_{1}\right)=\tilde{m}$ for any $c_{1} \in\left(\delta^{\tilde{m}}, \delta^{\tilde{m}-1}\right)$, we can conclude that the optimal commitment level is equal to $1-\beta_{2} \delta^{\tilde{m}}$, as claimed in the lemma.

Step 6. We have shown that in UCE, player 1 commits to $\psi_{1}$, as defined in the statement of the lemma. Corollary 3.4.3 implies immediate and efficient agreement. Moreover, the payoff to player 1 is $\pi_{1}\left(\psi_{1}\right)$, as desired.

We will now elaborate on the most important implications of the above result and its proof, and derive a number of corollaries. 
To begin with, knowing the payoff function $\pi_{1}($.$) and the UCE commitment level \psi_{1}$, we can explicitly state the UCE payoff for player 1 , which we denote by $\varphi_{1}$.

Corollary 3.4.5 Player 1's UCE payoff $\varphi_{1}$ is given by

$$
\varphi_{1}= \begin{cases}\beta_{1}+\beta_{2} \delta^{\tilde{m}}-\beta_{1} \beta_{2} \delta^{\tilde{m}+1} & \text { if } \delta^{\tilde{m}} \geq \frac{1}{2-\delta \beta_{1}} \\ 1-\beta_{2} \delta^{\tilde{m}} & \text { otherwise }\end{cases}
$$

Given that the recognition probabilities of both players and the discount factor all lie strictly between zero and one, we can see from the above expression for $\varphi_{1}$ that $\beta_{1}<\varphi_{1}<1$. While player 1 strictly benefits from his commitment power, he never obtains the entire pie. This is intuitively clear since with the commitment device at hand here, player 2 can always choose to hold out until $\tau\left(c_{1}\right)$, in which case some strictly positive surplus will be left over.

Corollary 3.4.6 For any configuration of the recognition probabilities and the discount factor, player 1's UCE payoff strictly exceeds $\beta_{1}$, but falls short of the entire pie.

Another implication of the above proof is that $\psi_{1}>\frac{1}{2}$. To see this, suppose by way of contradiction that $\psi_{1} \leq \frac{1}{2}$. Since $\frac{1}{2}+\beta_{2} \delta^{\tau\left(\frac{1}{2}\right)}<1$, the arguments in Steps 1 and 3 of the proof above imply that $\psi_{1}=\delta^{\tilde{m}}$. But by definition of $\psi_{1}$, we have that $\psi_{1}=\delta^{\tilde{m}}$ only if $\delta^{\tilde{m}} \geq \frac{1}{2-\delta \beta_{1}}$. It follows that $\frac{1}{2} \geq \frac{1}{2-\delta \beta_{1}}$, a contradiction to the assumption that $\delta$ and $\beta_{1}$ are strictly positive.

Corollary 3.4.7 The optimal commitment level $\psi_{1}$ is strictly greater than one half, irrespective of the choices of $\delta$ and $\beta$. Moreover, since in UCE agreement is reached with the commitment effective, the UCE payoff of player 1 is greater than one half.

Theorem 3.4.4 also implies that in equilibrium, player 1's commitment is chosen high enough so that disagreement in round $t=0$ would lead to delay until the expiry of the commitment. To see this, notice that from the definition of $\tilde{m}$, we have $\delta^{\tilde{m}}>\frac{\delta}{1-\beta_{2} \delta}$. Since $c_{1} \geq \delta^{\tilde{m}}$ by the proof of the above theorem, it holds that $c_{1}+\beta_{2} \delta c_{1}>\delta$. Given that $\delta c_{1} \leq \delta^{\tau\left(c_{1}\right)}$, the corollary follows.

Corollary 3.4.8 In UCE, the sum of players' aspirations exceeds $\delta^{t}$ at any time $t=$ $1, \ldots, \tau(\psi)$. Thus, $a_{1}\left(\psi_{1}\right)=0$.

Player 1 makes a commitment which is low enough to deter player 2 from holding out until time $\tau\left(c_{1}\right)$. But the previous corollary means that, loosely speaking, the optimal commitment is not "much" lower then a commitment which would lead to delay. More precisely, delay would result from any commitment $c_{1}$ such that $c_{1}>\varphi_{1}$. To see this, notice that by Theorems 3.4.4 and 3.3.8 the maximum payoff for player 1 in an SPBE with immediate agreement of a game $G\left(c_{1}\right)$ is equal to $\varphi_{1}$. This payoff is attained when $c_{1}=\psi_{1}$. Now consider a game $G\left(c_{1}\right)$ for some $c_{1}>\varphi_{1}$. Suppose that agreement is reached at $t=0$ in this game. Since the commitment $c_{1}$ is effective at time $t=0$, the payoff to player 1 must be at least $c_{1}$. But since $c_{1}>\varphi_{1}$, we have a contradiction. 
Corollary 3.4.9 If $c_{1}>\varphi_{1}$, then no SPBE of the game $G\left(c_{1}\right)$ involves agreement at time $t=0$.

The two previous corollaries indicate that a rather small deviation from the optimal level of commitment can lead into a subgame with equilibrium delay. While the equilibrium prediction in the model at hand does imply immediate agreement, this property is more fragile than in standard Rubinstein bargaining games, where immediate agreement results in every subgame.

We have pointed out that in UCE an agreement is reached while player 1's commitment is effective, therefore we have that $\varphi_{1} \geq \psi_{1}$. In the case where $c_{1}=\delta^{\tilde{m}}$, there is some "friction" between the commitment and the resulting payoff, which arises from the fact that $\delta^{\tau\left(c_{1}\right)}$ changes in a "stepwise" fashion with $c_{1}$. However, in the limit as $\delta \rightarrow 1$, these steps become ever smaller and the said friction vanishes as $c_{1}-\delta^{\tau\left(c_{1}\right)} \rightarrow 0$. It is not surprising, then, that $\psi_{1}$ and $\varphi_{1}$ do converge to the same limit. More formally, the UCE commitment level $\psi_{1}$ has been defined as equal to either $\delta^{\tilde{m}}$ or $1-\beta_{2} \delta^{\tilde{m}}$. But both of these terms are arbitrarily close to $\frac{1}{1+\beta_{2}}$ when $\delta$ is sufficiently close to one. Similarly, the term $\beta_{1}+\beta_{2} \delta^{\tilde{m}}-\beta_{1} \beta_{2} \delta^{\tilde{m}-1}$ used in the expression for $\varphi_{1}$ also converges to that same limit.

Theorem 3.4.10 In the limit as $\delta \rightarrow 1$, the UCE division of the surplus converges to $\left(\bar{\varphi}_{1}, 1-\right.$ $\left.\bar{\varphi}_{1}\right)=\left(\frac{1}{1+\beta_{2}}, \frac{\beta_{2}}{1+\beta_{2}}\right)$.

The intuition behind the limit result is as follows. If $\delta$ is close enough to one, the surplus which remains at the moment of truth is nearly equal to the commitment. Thus, player 2 can obtain $\beta_{2}$ times the committed amount by delaying agreement until the moment of truth. Anticipating this, player 1 chooses the commitment just low enough to make player 2 willing to enter into an agreement immediately. Hence, the surplus is divided in the proportion $1: \beta_{2}$.

Let us suppose that $\beta_{2}$ is very high. Given that the distribution of proposal power is very favorable to player 2, can player 1 compensate for his weakness if he is given the possibility to commit? Theorem 3.4.10 implies that if $\delta$ and $\beta_{2}$ are both close to one, player 1 can obtain about one half of the pie. Hence, if $\delta$ is large, the power of one player to commit is just sufficient to compensate for the fact that proposal power is concentrated with the other player. If $\delta$ is small, however, the ability to commit is much more powerful than that. In fact, for $\delta$ close to zero, the player who is able to commit can obtain close to the entire pie even if his proposal power is arbitrarily small. We illustrate these findings with the following numerical example.

Example 3.4.11 Let $\beta_{2}=0.9$. Suppose first that the discount factor is very small, say, $\delta=0.1$. In that case, we have that $\tilde{m}=1$. The term $\frac{1}{2-\beta_{1} \delta}$ evaluates to $\frac{1}{1.99} \approx 0.5$. Since this is greater than $\delta^{\tilde{m}}=0.1$, the optimal commitment level is given by $\psi_{1}=1-\beta_{2} \delta^{\tilde{m}}=1-0.1 \times 0.9=$ 0.91. Also, $\varphi_{1}=0.91$.

Now suppose instead that $\delta=0.9$; then $\tilde{m}=6$. The term $\frac{1}{2-\beta_{1} \delta}$ evaluates to $\frac{1}{1.91} \approx 0.52$. Since this is smaller than $\delta^{\tilde{m}}=0.9^{6} \approx 0.53$, the optimal commitment level is given by $\psi_{1}=\delta^{\tilde{m}} \approx 0.53$. 
Consequently,

$$
\begin{aligned}
\varphi_{1} & \approx 0.1+0.9 \times 0.9^{6}-0.1 \times 0.9 \times 0.9^{7} \\
& \approx 0.1+0.9 \times 0.53-0.09 \times 0.47 \\
& \approx 0.1+0.477-0.0423 \\
& \approx 0.5347
\end{aligned}
$$

This example provides a numerical illustration of the effects of large vs. small discount factors when the proposal power is prejudiced in the advantage of one player.

We see that for small $\delta$, the implications of our notion of commitment are close to those which one would expect of an irrevocable and everlasting commitment. For large $\delta$, however, the type of commitment which we propose leads to different results. This pattern will be observed more often in the sequel of the paper, when we deal with games in which both players have access to the commitment device.

\subsection{Bargaining with two committed players}

In this section, we will consider a bargaining (sub-)game $G\left(c_{1}, c_{2}\right)$. In this game, the two players bargain according to the protocol specified earlier for the game $G\left(c_{1}\right)$. That is, in each round a proposer is determined by the probability distribution $\beta$. However, in the game $G\left(c_{1}, c_{2}\right)$, both players $k=1,2$ are committed and thus have the following utility functions.

$$
u_{k}\left(x_{k}, c_{k}, t\right)= \begin{cases}x_{k}-\lambda<0 & \text { if } x_{k}<c_{k} \leq \delta^{t} \\ x_{k} & \text { otherwise }\end{cases}
$$

To prepare the analysis, a number of concepts introduced for the game $G\left(c_{1}\right)$ need to be extended formally to the game $G\left(c_{1}, c_{2}\right)$. To begin with, we denote by $G\left(c_{1}, c_{2}, t\right)$ a subgame of the game $G\left(c_{1}, c_{2}\right)$ which starts with the move of nature at time $t$. An SPBE in such a subgame is an SPE which satisfies the two conditions set forth in Definition 3.3.1. We say that the SPBE payoffs of $G\left(c_{1}, c_{2}, t\right)$ are essentially unique if all SPBE of all subgames of the type $G\left(c_{1}, c_{2}, t\right)$ lead to the same payoffs. We denote these payoffs by $v_{1}^{t}\left(c_{1}, c_{2}\right)$ and $v_{2}^{t}\left(c_{1}, c_{2}\right)$, where we will sometimes omit the arguments $\left(c_{1}, c_{2}\right)$ if no confusion arises. The definition of aspiration is extended to the new setting as follows.

Definition 3.5.1 Suppose that for some $t \in \mathbb{N}_{0}$, the SPBE payoffs of $G\left(c_{1}, c_{2}, t+1\right)$ are essentially unique, and the corresponding payoff pair is $\left(v_{1}^{t+1}, v_{2}^{t+1}\right)$. Then, player $k$ 's aspiration at time $t$ under the commitments $\left(c_{1}, c_{2}\right)$ is

$$
\left.\alpha_{k}^{t}\left(c_{1}, c_{2}\right)=\min \left\{x_{k} \in[0,1] \mid u_{k}\left(x_{k}, c_{k}, t\right) \geq v_{k}^{t+1}\right)\right\}
$$

This definition implies that 


$$
\alpha_{k}^{t}\left(c_{1}, c_{2}\right)= \begin{cases}v_{k}^{t+1}\left(c_{1}, c_{2}\right) & \text { if } t \geq \tau\left(c_{k}\right) \\ \max \left\{c_{k}, v_{k}^{t+1}\left(c_{1}, c_{2}\right)\right\} & \text { otherwise }\end{cases}
$$

for both players $k=1,2$.

The above definition and the next two lemmas provide a backward-induction algorithm to find the SPBE payoffs in the game $G\left(c_{1}, c_{2}\right)$. Intuitively, Lemma 3.5.2 shows how to reason backwards from one round to the previous round, while Lemma 3.5.3 shows how to initialize the backward induction at the time when both commitments are void.

Lemma 3.5.2 Suppose that for some $t \in \mathbb{N}_{0}$, the SPBE payoffs of $G\left(c_{1}, c_{2}, t+1\right)$ are essentially unique and equal to $\left(v_{1}^{t+1}, v_{2}^{t+1}\right)$. Then, the SPBE payoffs of $G\left(c_{1}, c_{2}, t\right)$ are essentially unique as well and given by

$$
v_{k}^{t}\left(c_{1}, c_{2}\right)= \begin{cases}\alpha_{k}^{t}\left(c_{1}, c_{2}\right)+\beta_{k}\left(\delta^{t}-\alpha_{k}^{t}\left(c_{1}, c_{2}\right)-\alpha_{l}^{t}\left(c_{1}, c_{2}\right)\right) & \text { if } \alpha_{k}^{t}\left(c_{1}, c_{2}\right)+\alpha_{l}^{t}\left(c_{1}, c_{2}\right) \leq \delta^{t} \\ v_{k}^{t+1} & \text { otherwise }\end{cases}
$$

for any player $k=1,2$ and $l \neq k$.

This result follows from the proof of Lemma 3.3.7.

Lemma 3.5.3 Let $\bar{t}=\max \left\{\tau\left(c_{1}\right), \tau\left(c_{2}\right)\right\}$. In any subgame $G\left(c_{1}, c_{2}, \bar{t}\right)$, player $k$ 's $(k=i, j)$ expected $S P B E$ payoff is equal to $\beta_{k} \delta^{\bar{t}}$.

This statement corresponds to Lemma 3.3.3, and ensures the essential uniqueness of SPBE of $G\left(c_{1}, c_{2}, \bar{t}\right)$. In previous sections, the only player who could commit was referred to as player 1. This labeling has been arbitrary. Thus, we can define $\psi_{k}$ as the optimal level of commitment which player $k=1,2$ chooses in the game in which he is the only player who can commit. In a similar way, let $\pi_{k}\left(c_{k}\right)$ be the SPBE payoff in the bargaining subgame $G\left(c_{k}\right)$, and define $\varphi_{k}=\pi_{k}\left(\psi_{k}\right)$. For the rest of the current section, we will use $i$ to denote the player with the highest commitment level. That is, we assume without loss of generality that $c_{i} \geq c_{j}$. Let us first consider a subgame $G\left(c_{i}, c_{j}, \tau\left(c_{i}\right)\right)$. That is, we are interested in a subgame which starts at the time when the higher of the two commitments has become void. In such a subgame, only player $j$ remains committed. Therefore, it seems intuitive that such a subgame is equivalent to a bargaining subgame with one committed player, as we have analyzed in Section 3. This idea is stated more formally in the following lemma.

Lemma 3.5.4 In any subgame $G\left(c_{i}, c_{j}, \tau\left(c_{i}\right)\right)$, the SPBE payoffs are equal to $\delta^{\tau\left(c_{i}\right)} \pi_{j}\left(c_{j} \delta^{-\tau\left(c_{i}\right)}\right)$.

Proof: Consider the backward-induction algorithm described by Equations (12)-(13) at any round $t \geq \tau\left(c_{i}\right)$. At such a round $t$, the Equations (12)-(13) reduce to 


$$
\begin{gathered}
\alpha_{i}^{t}=v_{i}^{t+1} \\
\alpha_{j}^{t}= \begin{cases}v_{j}^{t+1} & \text { if } t \geq \tau\left(c_{j}\right) \\
\max \left\{c_{j}, v_{j}^{t+1}\right\} & \text { otherwise }\end{cases} \\
v_{k}^{t}= \begin{cases}\alpha_{k}^{t}+\beta_{k}\left(\delta^{t}-\alpha_{i}^{t}-\alpha_{j}^{t}\right) & \text { if } \alpha_{i}^{t}+\alpha_{j}^{t} \leq \delta^{t} \\
v_{k}^{t+1} \text { otherwise, } k=i, j & \end{cases}
\end{gathered}
$$

These equations correspond to Equations (9)-(11) which describe the backward-induction algorithm in the game with one committed player. We can view each step of this algorithm as a function $f\left(v^{t+1}, c_{j}, \delta^{t}\right)$ which determines $v^{t}$. One can easily verify from Equations (9)-(11) that this function is linearly homogenous of degree one, that is, we have $f\left(\kappa v^{t+1}, \kappa c_{j}, \kappa \delta^{t}\right)=$ $\kappa f\left(v^{t+1}, c_{j}, \delta^{t}\right)$ for any $\kappa>0$. Moreover, we use the fact that $\tau\left(c_{j} \delta^{t}\right)=\tau\left(c_{j}\right)+t$ for $t \in \mathbb{N}_{0}$. One implication of this fact is that the backward induction from $\tau\left(c_{j}\right)$ to $\tau\left(c_{i}\right)$ takes as many iterative steps as the backward induction from $\tau\left(\tilde{c}_{j}\right)$ to round zero, where $\tilde{c}_{j}=c_{j} \delta^{-\tau\left(c_{i}\right)}$. Since in Section 3, the size of the initial surplus was normalized to one without loss of generality, the claim follows.

The backward-induction algorithm described by Equations (12)-(13) finds the essentially unique SPBE payoffs in any bargaining (sub-)game $G\left(c_{i}, c_{j}\right)$. We will write these payoffs to players $i$ and $j$, respectively, as $\omega_{i}\left(c_{i}, c_{j}\right)$ and $\omega_{j}\left(c_{i}, c_{j}\right)$.

Lemma 3.5.4 has two important implications, which we state in the following two corollaries. In Theorem 3.4.4 we have found the optimal payoff for the only committed player. Since a subgame $G\left(c_{i}, c_{j}, \tau\left(c_{i}\right)\right)$ is indeed a game with one committed player, we have the following corollary.

Corollary 3.5.5 In a subgame $G\left(c_{i}, c_{j}, \tau\left(c_{i}\right)\right)$, player $j$ 's SPBE payoff is at most $\varphi_{j} \delta^{\tau\left(c_{i}\right)}$.

Given any commitment level $c_{i}$ chosen by player $i$, player $j$ may adopt a strategy which involves making the commitment $\psi_{j} \delta^{\tau\left(c_{i}\right)}$ and not entering into any agreement before time $\tau\left(c_{i}\right)$.

Corollary 3.5.6 It holds that $\omega_{j}\left(c_{i}, \psi_{j} \delta^{\tau\left(c_{j}\right)}\right) \geq \varphi_{j} \delta^{\tau\left(c_{i}\right)}$.

Intuitively, given any commitment level $c_{i}$ of player $i$, player $j$ has the option to hold out until time $\tau\left(c_{i}\right)$ and then play a subgame in which he is the only committed player. In such a subgame, the analysis of Section 3 is applicable, and player $j$ 's commitment and proposal power combined will allow him to capture a share of $\varphi_{j}$ of the remaining surplus. However, this surplus has shrunk by a factor $\delta^{\tau\left(c_{i}\right)}$ in the meantime. There is a trade-off between the advantageous position of the only committed player and the cost of delay incurred to acquire this position. 
We will now provide some illustration of the backward induction procedure in the game at hand, in which the notion of $c_{i}$ and $c_{j}$ being close to each other plays a crucial role. Let us suppose that $c_{j}$ is sufficiently small so that $c_{j}+\beta_{i} \delta^{\tau\left(c_{j}\right)} \leq 1$. Then, we can define

$$
a_{j}\left(c_{j}\right)=\max \left\{z \in \mathbb{N}_{0} \mid \delta^{z} \geq c_{j}+\beta_{i} \delta^{\tau\left(c_{j}\right)}\right\}
$$

Definition 3.5.7 We say that the commitments $c_{i}$ and $c_{j}$ are close to each other if $a_{j}\left(c_{j}\right)<$ $\tau\left(c_{i}\right)$.

Lemma 3.5.8 Suppose $c_{j}+\beta_{i} \delta^{\tau\left(c_{j}\right)} \leq 1$. Then, $c_{i}$ and $c_{j}$ are close to each other if $c_{j}>\varphi_{j} \delta^{\tau\left(c_{i}\right)}$.

This statement follows from Corollary 3.4.9.

Let us suppose first that the commitments are not close to each other. We can then illustrate the situation with the following time-lines. Lemma 3.5.3 gives the payoffs in a subgame starting in round $\tau\left(c_{j}\right)$, allowing us to initialize the backward induction specified in Equations (12)-(13). Applying these equations iteratively, we find the following. Before round $\tau\left(c_{j}\right)$, the aspirations are $\beta_{i} \delta^{\tau\left(c_{i}\right)}$ and $c_{j}$. Thus, no agreement is possible between rounds $a_{j}\left(c_{j}\right)$ and $\tau\left(c_{j}\right)$, as indicated by the dotted line segment. But an agreement can be reached between time $\tau\left(c_{i}\right)$ and time $a_{j}\left(c_{j}\right)$. But going backwards from $\tau\left(c_{i}\right)$ to $\tau\left(c_{i}\right)-1$, player $i$ 's aspiration jumps to $c_{i}$ while player $j$ 's aspiration is equal to $\delta^{\tau\left(c_{i}\right)} \pi_{j}\left(c_{j} \delta^{-\tau\left(c_{i}\right)}\right)$ as by Lemma 3.5.4. If $c_{i}+\delta^{\tau\left(c_{i}\right)} \pi_{j}\left(c_{j} \delta^{-\tau\left(c_{i}\right)}\right)>1$, then no agreement can be reached before time $\tau\left(c_{i}\right)$, as indicated on the first time-line below.

$$
t=0 \quad \tau\left(c_{i}\right) \quad a_{j}\left(c_{j}\right) \quad \tau\left(c_{j}\right)
$$

If, to the contrary, it holds that $c_{i}+\delta^{\tau\left(c_{i}\right)} \pi_{j}\left(c_{j} \delta^{-\tau\left(c_{i}\right)}\right) \leq 1$, then we find

$$
b\left(c_{i}, c_{j}\right):=\max \left\{z \in \mathbb{N}_{0} \mid \delta^{z} \geq c_{i}+\delta^{\tau\left(c_{i}\right)} \pi_{j}\left(c_{j} \delta^{-\tau\left(c_{i}\right)}\right)\right\}
$$

as the latest round in which agreement can be reached before $\tau\left(c_{i}\right)$ - a situation depicted in the next time-line.

$$
t=\underset{0}{0} \quad b\left(c_{i}, c_{j}\right) \quad \tau\left(c_{i}\right) \quad a_{j}\left(c_{j}\right) \quad \tau\left(c_{j}\right)
$$

Now consider the case where $c_{i}$ and $c_{j}$ are close to each other. In this case, it is not possible to reach agreement at round $a_{j}\left(c_{j}\right)$ since player $i$ 's aspiration has jumped up to $c_{i}$ already before the backward induction procedure reached round $a_{j}\left(c_{j}\right)$. Hence agreement is impossible 


$$
t=0 \quad \tau\left(c_{i}+c_{j}\right)-1 \quad a_{j}\left(c_{j}\right) \quad \tau\left(c_{i}\right) \quad \tau\left(c_{j}\right)
$$

between time $\tau\left(c_{i}+c_{j}\right)-1$ (the latest point in time when the surplus is at least $\left.c_{i}+c_{j}\right)$ and time $\tau\left(c_{j}\right)$, as shown in the following time-line. Of course, if $c_{i}+c_{j}>1$ and $c_{i}$ and $c_{j}$ are close to each other, no agreement can be reached at all until round $\tau\left(c_{j}\right)$.

To end the section, we provide two auxiliary results which we will use in the sequel of the paper. The first statement below says that in SPBE, the lower of the two commitments is effective at the time of the agreement unless the sum of commitments exceeds one.

Lemma 3.5.9 If $c_{i}+c_{j} \leq 1$, then $\omega_{j}\left(c_{i}, c_{j}\right) \geq c_{j}$.

Proof: Suppose not. Then, there is $\left(c_{i}, c_{j}\right)$ such that $c_{i}+c_{j} \leq 1$ and $\omega_{j}\left(c_{i}, c_{j}\right)<c_{j}$. By definition of $\omega_{j}($.$) , this function gives the payoff arising from some SPBE of the game G\left(c_{i}, c_{j}\right)$. But in an SPBE, player $j$ does not agree to less than $c_{j}$ before time $\tau\left(c_{j}\right)$. Hence, an SPBE of $G\left(c_{i}, c_{j}\right)$ must involve delay until at least round $\tau\left(c_{j}\right)$. By Lemma 3.5.2, we have $\alpha_{i}^{t}+\alpha_{j}^{t}>\delta^{t}$ for all $t=0,1, \ldots, \tau\left(c_{j}\right)-1$. Notice that the assumption $c_{i} \geq c_{j}$ and the supposition $c_{i}+c_{j} \leq 1$ imply that $c_{j} \leq \frac{1}{2}$. But since $\delta^{\tau\left(c_{j}\right)}<c_{j}$ and $\beta_{i}<1$, this in turn implies $c_{j}+\beta_{i} \delta^{\tau\left(c_{j}\right)} \leq 1$. Thus, $a_{j}\left(c_{j}\right)$ is well-defined and agreement can be reached in that round unless $c_{i}$ and $c_{j}$ are close to each other. Indeed, suppose now that $c_{i}$ and $c_{j}$ are close to each other. But then the aspirations in round $\tau\left(c_{i}+c_{j}\right)-1$ are equal to the commitments $c_{i}$ and $c_{j}$. Since $c_{i}+c_{j} \leq 1$, we have that $\tau\left(c_{i}+c_{j}\right) \geq 1$ and thus agreement can be reached at some round $t \in \mathbb{N}_{0}$, the desired contradiction.

In particular, if the commitments are close to each other and sum up to exactly one, then we have $\alpha_{i}^{0}+\alpha_{j}^{0}=1$ : The aspirations at $t=0$ are equal to the commitments and sum up to the total surplus. By the conditions set forth in the definition of an SPBE, agreement must be reached on the division of the surplus according to the commitments.

Corollary 3.5.10 If $c_{i}+c_{j}=1$ and $c_{i}$ and $c_{j}$ are close to each other, then the SPBE payoffs are equal to $c_{i}$ and $c_{j}$.

\subsection{Optimal commitment for two players}

In this section, we turn to the equilibrium analysis of games where both players can commit before bargaining starts. We will consider one game in which the players make their commitments sequentially, and one in which the commitments are chosen simultaneously. Recall that the SPBE payoffs in a bargaining subgame with a given pair of commitments $\left(c_{1}, c_{2}\right)$ are given by $\omega_{1}\left(c_{1}, c_{2}\right)$ and $\omega_{2}\left(c_{1}, c_{2}\right)$. Hence, we are effectively considering a game in which each player's action is to choose a commitment from the interval $[0,1]$ and the payoff functions are given by $\omega_{1}\left(c_{1}, c_{2}\right)$ and $\omega_{2}\left(c_{1}, c_{2}\right)$. 
In this section, we abandon the notational convention that $c_{i} \geq c_{j}$. After giving a formal definition of a (unique) best-response, we will state a number of auxiliary lemmas, which will be needed to prove the main results of this section.

Definition 3.6.1 We say that the commitment $\bar{c}_{j}$ is a best-response to the commitment $\bar{c}_{i}$ of player $i \neq j$ if it holds that $\omega_{j}\left(\bar{c}_{i}, \bar{c}_{j}\right) \geq \omega_{j}\left(\bar{c}_{i}, c_{j}\right)$ for all $c_{j} \in[0,1]$. If the inequality holds strictly for all $c_{j} \in[0,1]$, then we say that $\bar{c}_{j}$ is the unique best-response to $\bar{c}_{i}$.

The next lemma claims that there is some intermediate range of commitments for player $i$ so that player $j$ 's best-response is to commit to the exact complement.

Lemma 3.6.2 Suppose that $c_{i} \leq 1-\varphi_{j} \delta^{\tau\left(c_{i}\right)}$ and $c_{i} \geq \varphi_{i} \delta^{\tau\left(1-c_{i}\right)}$. Then, it is a best-response for player $j$ to choose the commitment level $c_{j}=1-c_{i}$. The best-response is unique if the inequalities hold strictly.

Proof: Indeed, suppose that $c_{i} \leq 1-\varphi_{j} \delta^{\tau\left(c_{i}\right)}$ and $c_{i} \geq \varphi_{i} \delta^{\tau\left(1-c_{i}\right)}$. By Lemma 3.5.8, $c_{i}$ and $1-c_{i}$ are close to each other, so that $\omega_{i}\left(c_{i}, 1-c_{i}\right)=c_{i}$ and $\omega_{j}\left(c_{i}, 1-c_{i}\right)=1-c_{i}$. We want to show that no choice of commitment $c_{j}$ gives player $j$ a strictly higher payoff than $1-c_{i}$. If player $j$ chooses some $c_{j}$ such that the SPBE of $G\left(c_{i}, c_{j}\right)$ involves agreement before $\tau\left(c_{i}\right)$, the payoff to player $j$ is bounded above by $1-c_{i}$. But if $j$ chooses $c_{j}$ such that delay occurs until round $\tau\left(c_{i}\right)$, then by Corollary 3.5.5, his payoff is bounded above by $\varphi_{j} \delta^{\tau\left(c_{i}\right)} \leq 1-c_{i}$, as desired.

In the next lemma, we consider the case where a player has chosen a commitment which is too high to fall into the range of commitments to which Lemma 3.6.2 applies. In that case, we show that it is optimal for the other player to hold out and delay agreement until this high commitment has expired.

Lemma 3.6.3 Suppose that $c_{i}>1-\varphi_{j} \delta^{\tau\left(c_{i}\right)}$. Then, the unique best-response for player $j$ is to choose the commitment level $c_{j}=\psi_{j} \delta^{\tau\left(c_{i}\right)}$.

Proof: Suppose indeed that $1-c_{i}<\varphi_{j} \delta^{\tau\left(c_{i}\right)}$. Suppose first that player $j$ chooses some $c_{j}$ such that in the essentially unique SPBE of $G\left(c_{i}, c_{j}\right)$, agreement is reached before $\tau\left(c_{i}\right)$. Since the commitment $c_{i}$ is effective at the time of this agreement, the payoff to player $j$ in this SPBE is at most $1-c_{i}$. But by Corollary 3.5.6, he can obtain $\varphi_{j} \delta^{\tau\left(c_{i}\right)}>1-c_{i}$ by committing to $\psi_{j} \delta^{\tau\left(c_{i}\right)}$. Thus, a commitment level $c_{j}$ can only be a best-response for player $j$ if in an SPBE of $G\left(c_{i}, c_{j}\right)$, no agreement is made before round $\tau\left(c_{i}\right)$. But from Theorem 3.4.4 and Lemma 3.5.4, it follows that $\psi_{j} \delta^{\tau\left(c_{i}\right)}$ is uniquely optimal among all commitments $c_{j}$ which do lead to a delay until round $\tau\left(c_{i}\right)$ in an $\mathrm{SPBE}$ of $G\left(c_{i}, c_{j}\right)$.

Lemma 3.6.4 below says the following. If player $i$ makes a "high" commitment in the sense of Lemma 3.6.3 above, and if player $j$ responds optimally to this by delaying agreement, then a unilateral deviation to a commitment of zero would be profitable for player $i$.

Lemma 3.6.4 If $c_{i}$ is such that $1-c_{i}<\varphi_{j} \delta^{\tau\left(c_{i}\right)}$, then $\omega_{i}\left(c_{i}, \psi_{j} \delta^{\tau\left(c_{i}\right)}\right)<\omega_{i}\left(0, \psi_{j} \delta^{\tau\left(c_{i}\right)}\right)$. 
Proof: Suppose that the pair of commitments $\left(c_{i}, c_{j}\right)$ is such that $c_{j}=\psi_{j} \delta^{\tau\left(c_{i}\right)}$ and $1-c_{i}<$ $\varphi_{j} \delta^{\tau\left(c_{i}\right)}$. In the SPBE of a subgame $G\left(c_{i}, c_{j}, \tau\left(c_{i}\right)\right)$, the payoffs to players $i$ and $j$, respectively, will be $\left(1-\varphi_{j}\right) \delta^{\tau\left(c_{i}\right)}$ and $\varphi_{j} \delta^{\tau\left(c_{i}\right)}$. But since $1<c_{i}+\varphi_{j} \delta^{\tau\left(c_{i}\right)}$, no agreement can be reached in an SPBE at any $t<\tau\left(c_{i}\right)$. Thus, these payoffs are also the payoffs in the SPBE of the entire bargaining game $G\left(c_{i}, c_{j}\right)$. It holds that $c_{j}=\psi_{j} \delta^{\tau\left(c_{i}\right)} \leq \psi_{j} \delta<\psi_{j}$. The first inequality follows because $c_{i}>0$ and thus $\tau\left(c_{i}\right) \geq 1$. (Suppose to the contrary that $c_{i}=0$. Then, the supposition that $1-c_{i}<\varphi_{j} \delta^{\tau\left(c_{i}\right)}$ reduces to $1<\varphi_{j}$, a contradiction.) The second inequality follows because $\delta<1$. Now suppose player $i$ deviates to the commitment $\hat{c}_{i}=0$, while player $j$ remains at his commitment level $c_{j}<\psi_{j}$. The induced bargaining game $G\left(0, c_{j}\right)$ is equivalent to the game with one committed player, as analyzed in Section 3. Since $c_{j} \leq \psi_{j} \delta<\psi_{j}$, Theorem 3.3.8 implies that an efficient agreement is reached immediately in SPBE of $G\left(0, c_{j}\right)$. But, by Corollary 3.5.6, it holds that the payoff to player $j$ in that agreement is at most $\varphi_{j}$. Thus, the payoff to player $i$ is at least $1-\varphi_{j}$. But $1-\varphi_{j}>\left(1-\varphi_{j}\right) \delta^{\tau\left(c_{i}\right)}$, so the deviation from $c_{i}$ to $\hat{c}_{i}$ is profitable for player $i$, as desired.

For player $i=1,2$, we now define a particular commitment level $\eta_{i}$ as follows.

$$
\eta_{i}=\max \left\{c_{i} \mid c_{i}+\varphi_{j} \delta^{\tau\left(c_{i}\right)} \leq 1\right\}
$$

Loosely speaking, $\eta_{i}$ is the maximal commitment level which is not "high" in the sense of Lemma 3.6.3 above. It is important to realize that $\eta_{i} \geq \frac{1}{2}$. In order to see this, suppose to the contrary that $\eta_{i}<\frac{1}{2}$. Then, it follows that $\frac{1}{2}+\varphi_{j} \delta^{\tau\left(\frac{1}{2}\right)}>1$. But $\varphi_{j}<1$ and $\delta^{\tau\left(\frac{1}{2}\right)}<\frac{1}{2}$, a contradiction.

Remark 3.6.5 The fact that $\eta_{i} \geq \frac{1}{2}$ implies that $\varphi_{i} \delta^{\tau\left(1-\eta_{i}\right)}<\frac{1}{2} \leq \eta_{i}$. Thus, the commitments $\left(\eta_{i}, 1-\eta_{i}\right)$ are close to each other.

The next lemma is the last auxiliary which we need to prove the main results of the section. The lemma says that if player $i$ commits to less than $\eta_{i}$, then player $j$ can always obtain a greater payoff than $1-\eta_{i}$ by choosing an appropriate commitment.

Lemma 3.6.6 Suppose that $c_{i}<\eta_{i}$. Then, there exists $\varepsilon>0$ sufficiently small so that $\omega_{j}\left(c_{i}, 1-\right.$ $\left.\eta_{i}+\varepsilon\right)>1-\eta_{i}$.

Proof: Suppose to the contrary that there is a pair of commitments $\left(\bar{c}_{i}, \bar{c}_{j}\right)$ such that $\bar{c}_{i}<\eta_{i}$ and $\bar{c}_{j}=1-\eta_{i}+\varepsilon$, but $\omega_{j}\left(\bar{c}_{i}, \bar{c}_{j}\right) \leq 1-\eta_{i}<\bar{c}_{j}$. We will derive a contradiction for sufficiently small $\varepsilon>0$. Before time $\tau\left(\bar{c}_{j}\right)$, player $j$ does not agree to less than $\bar{c}_{j}$ in SPBE. Thus, in any SPBE of $G\left(\bar{c}_{i}, \bar{c}_{j}\right)$, there must be delay until at least time $\tau\left(\bar{c}_{j}\right)$. Let us consider first the case where $\bar{c}_{i}>\bar{c}_{j}$. In SPBE, the smaller of two commitments expires only if $\bar{c}_{i}+\bar{c}_{j}>1$ (see Lemma 3.5.9). This inequality is equivalent to $\bar{c}_{i}+1-\eta_{i}+\varepsilon>1$. Since $\eta_{i}>\bar{c}_{i}$, we obtain the desired contradiction for sufficiently small $\varepsilon>0$. Now consider the case where $\bar{c}_{j} \geq \bar{c}_{i}$. Since there is delay until round $\tau\left(\bar{c}_{j}\right)$, and since $\delta^{\tau\left(c_{j}\right)}<\bar{c}_{j}$, the payoff to player $i$ in a subgame $G\left(c_{i}, c_{j}, \tau\left(c_{j}\right)\right)$ is strictly smaller than $\bar{c}_{j}=1-\eta_{i}+\varepsilon$. In terms of the aspirations at time $\bar{t}=\tau\left(c_{j}\right)-1$, we have $\alpha_{i}^{\bar{t}}<\bar{c}_{j}=1-\eta_{i}+\varepsilon$ and $\alpha_{j}^{\bar{t}}=\bar{c}_{j}=1-\eta_{i}+\varepsilon$. Summing up the aspirations yields 
$\alpha_{i}^{\bar{t}}+\alpha_{i}^{\bar{t}}<2 \bar{c}_{j}=2\left(1-\eta_{i}+\varepsilon\right)$. Using the fact that $\eta_{i} \geq \frac{1}{2}$, we find $\alpha_{i}^{\bar{t}}+\alpha_{i}^{\bar{t}}<1+2 \varepsilon$. But if $\varepsilon>0$ is sufficiently small, then $\alpha_{i}^{\bar{t}}+\alpha_{i}^{\bar{t}} \leq 1$, in which case agreement is reached in SPBE in round $\bar{t}$. Since $\bar{t}<\tau\left(\bar{c}_{j}\right)$, we have obtained the desired contradiction.

We will now turn to the proof of the main results, for which the previous lemmas will be crucial. We begin with the case in which players choose their commitments sequentially. More formally, by the sequential commitment game, we mean the game in which first player 1 chooses a commitment level $c_{1} \in[0,1]$, then player 2 chooses a commitment level $c_{2} \in[0,1]$, and then the bargaining game $G\left(c_{1}, c_{2}\right)$ is played. Let $\left(\bar{s}_{1}, \bar{s}_{2}\right)$ be a strategy profile in the sequential commitment game, and suppose that under this strategy profile, the players choose commitments $\left(\bar{c}_{1}, \bar{c}_{2}\right)$. The profile $\bar{s}$ is a sequential commitment equilibrium (SQCE) if it satisfies the following conditions.

1. The restriction of $\bar{s}$ to the bargaining subgame $G\left(\bar{c}_{1}, \bar{c}_{2}\right)$ is an SPBE in that bargaining game.

2. It holds that $\omega_{2}\left(\bar{c}_{1}, \bar{c}_{2}\right) \geq \omega_{2}\left(\bar{c}_{1}, c_{2}\right)$ for all $c_{2} \in[0,1]$.

3. There is no $\left(\tilde{c}_{1}, \tilde{c}_{2}\right) \in[0,1] \times[0,1]$ such that $\omega_{2}\left(\tilde{c}_{1}, \tilde{c}_{2}\right) \geq \omega_{2}\left(\tilde{c}_{1}, c_{2}\right)$ for all $c_{2} \in[0,1]$, and $\omega_{1}\left(\tilde{c}_{1}, \tilde{c}_{2}\right)>\omega_{1}\left(\bar{c}_{1}, \bar{c}_{2}\right)$.

The second condition above means that player 2 responds optimally to player 1's commitment choice in any SQCE. The third condition means that player 1 chooses his commitment optimally, anticipating the response of player 2. The next theorem claims that there is a unique SQCE division of the surplus, which is immediately agreed upon.

Theorem 3.6.7 In any SQCE of the sequential commitment game, an immediate and efficient agreement is reached on the division $\left(\eta_{1}, 1-\eta_{1}\right)$.

Proof: Suppose first that in some SQCE player 1 chooses $c_{1}>\eta_{1}$. By Lemma 3.6.3, player 2 will choose $c_{2}=\psi_{2} \delta^{\tau\left(c_{1}\right)}$ in response. The resulting payoffs for player 1 will be $\left(1-\varphi_{2}\right) \delta^{\tau\left(c_{1}\right)}$. But if player 1 had chosen a commitment of zero, then player 2's best-response would have been to choose $\psi_{2}$, which would give player 1 the payoff of $1-\varphi_{2}>\left(1-\varphi_{2}\right) \delta^{\tau\left(c_{1}\right)}$. Hence, we have shown that in SQCE, player 1 chooses $c_{1}$ such that $c_{1} \leq \eta_{1}$.

Suppose that player 1 chooses $c_{1}<\eta_{1}$. By Lemma 3.6.6, player 1's payoff is then strictly less than $\eta_{1}$. However, he can obtain a payoff arbitrarily close to $\eta_{1}$. More precisely, if player 1 chooses $c_{1}=\eta_{1}-\varepsilon$ for some sufficiently small $\varepsilon>0$, then by Lemma 3.6.2, player 2 will choose $c_{2}=1-c_{1}$ in response and thus player 1 can realize a payoff of $c_{1}=\eta_{1}-\varepsilon$. Thus, a commitment $c_{1}$ so that $c_{1}<\eta_{1}$ can never be optimal.

We have now shown that $c_{1}=\eta_{1}$ in an SQCE. By Lemma 3.6.2, committing to $1-\eta_{1}$ is a best-response for player 2 . Since $\eta_{1}$ and $1-\eta_{1}$ are close to each other, agreement is then reached immediately on the division $\left(\eta_{1}, 1-\eta_{1}\right)$. Finally, notice that if $\varphi_{2} \delta^{\tau\left(c_{1}\right)}=1-c_{1}$ but $\psi_{2}<\varphi_{2}$, then the best-response commitment $1-\eta_{1}$ is not unique. It would also be a best-response for player 2 to choose $\psi_{2} \delta^{\tau\left(c_{1}\right)}$. However, this would lead to aspirations of $c_{1}$ and $\varphi_{2} \delta^{\tau\left(c_{1}\right)}=1-c_{1}$ 
at round $t=0$. By the definition of SPBE, agreement would also be reached immediately on $\left(\eta_{1}, 1-\eta_{1}\right)$, as desired.

Intuitively, Theorem 3.6.7 can be understood as follows. Once player 1 has chosen $c_{1}$, player 2 always has the option to delay agreement until time $\tau\left(c_{1}\right)$ and then obtain the payoff $\varphi_{2} \delta^{\tau\left(c_{1}\right)}$. Player 1 chooses his commitment just low enough so that this option becomes unattractive for player 2. Qualitatively, the result is similar to that in the game with one committed player. In that game, the player who is not committed can hold out until the commitment expires. But after the commitment has expired, the bargaining powers are given by the recognition probabilities $\beta$. In the sequential commitment case, however, once round $\tau\left(c_{1}\right)$ is reached, player 2's bargaining power is given by $\varphi_{2}$ rather than just by his recognition probability $\beta_{2}$. In sum, we obtain a unique equilibrium prediction for the division of the surplus. The player who commits first has a first-mover advantage, but this advantage is weaker than the advantage of the committed over the un-committed player in the game with a single committed player. In the limit as $\delta \rightarrow 1$, we have that $c_{i}-\delta^{\tau\left(c_{i}\right)} \rightarrow 0$, and thus $\eta_{i}$ converges to the limit $\bar{\eta}_{i}=\frac{1}{1+\bar{\varphi}_{j}}$. But by Theorem 3.4.10, $\bar{\varphi}_{j}=\frac{1}{1+\beta_{i}}$. Consequently, we have the following limit result.

Theorem 3.6.8 In the limit as $\delta \rightarrow 1$, the SQCE division of the surplus converges to $\left(\bar{\eta}_{1}, 1-\right.$ $\left.\bar{\eta}_{1}\right)=\left(\frac{1+\beta_{1}}{2+\beta_{1}}, \frac{1}{2+\beta_{1}}\right)$.

We will now turn to the version of the model where both players choose their commitments simultaneously before bargaining starts. More formally, by the simultaneous commitment game, we mean the game in which first players 1 and 2 simultaneously choose commitment levels $c_{1} \in[0,1]$ and $c_{2} \in[0,1]$, and then the bargaining game $G\left(c_{1}, c_{2}\right)$ is played. Let $\left(\bar{s}_{1}, \bar{s}_{2}\right)$ be a strategy profile in the simultaneous commitment game, and $\left(\bar{c}_{1}, \bar{c}_{2}\right)$ the commitments chosen under that profile. The profile $\bar{s}$ is a simultaneous commitment equilibrium (SMCE) if its restriction to the bargaining subgame $G\left(\bar{c}_{1}, \bar{c}_{2}\right)$ is an SPBE, and if for $i=1,2$ and $j \neq i$, it holds that $\omega_{i}\left(\bar{c}_{i}, \bar{c}_{j}\right) \geq \omega_{i}\left(c_{i}, \bar{c}_{j}\right)$ for all $c_{i} \in[0,1]$. That is, each player's commitment is a best-response to the other player's commitment. Theorem 3.6.9 below shows that there is a range of divisions of the surplus which can be supported by SMCE. The endpoints of this range are given by the divisions which occur in the sequential commitment equilibrium, when either player acts as the first mover.

Theorem 3.6.9 A division $\left(x_{1}, x_{2}\right) \in \mathbb{R}_{+}^{2}$ of the surplus can be supported by an SMCE of the simultaneous commitment game if and only if $x_{1}+x_{2}=1$ and $x_{i} \geq 1-\eta_{j}$ for $i=1,2$ and $j \neq i$.

Proof: If: Consider a pair of commitments $\left(\bar{c}_{1}, \bar{c}_{2}\right)$ such that $\bar{c}_{1}+\bar{c}_{2}=1$ and $\bar{c}_{i} \geq 1-\eta_{j}$ for both $i=1,2$ and $j \neq i$. By definition of $\eta_{i}$, it follows that $\bar{c}_{i} \geq \varphi_{i} \delta^{\tau\left(\bar{c}_{j}\right)}$ for both $i=1,2$ and $j \neq i$. By Lemma 3.6.2, the commitments $\left(\bar{c}_{1}, \bar{c}_{2}\right)$ are best-responses to each other. Since they are also close to each other, Corollary 3.5.10 implies that payoffs in the SPBE of $G\left(\bar{c}_{1}, \bar{c}_{2}\right)$ will be $\left(\bar{c}_{1}, \bar{c}_{2}\right)$. 
Only If: We show first that in any SMCE, we have $c_{i} \leq \eta_{i}$ for both $i=1,2$. Suppose by way of contradiction that there is some SMCE in which the commitments are $\left(\bar{c}_{1}, \bar{c}_{2}\right)$ and $\bar{c}_{i}>\eta_{i}$ for some $i=1,2$. Then, by definition of $\eta_{i}$, it holds that $\bar{c}_{i}+\varphi_{j} \delta^{\tau\left(\bar{c}_{i}\right)}>1$ and by Lemma 3.6.3, it must hold that $\bar{c}_{j}=\psi_{j} \delta^{\tau\left(\bar{c}_{i}\right)}$ for $j \neq i$. But then, by Lemma 3.6.4, setting a commitment $\hat{c}_{i}=0$ is a profitable deviation for player $i$, the desired contradiction.

We show next that player $i=1,2$ obtains at least a payoff of $1-\eta_{j}$ in SMCE. Suppose by way of contradiction that there is some SMCE leading to payoffs $\left(\bar{u}_{1}, \bar{u}_{2}\right)$ such that $\bar{u}_{j}<1-\eta_{i}$ for some $j=1,2$ and $i \neq j$. Again, let $\left(\bar{c}_{1}, \bar{c}_{2}\right)$ be the commitment levels in the supposed SMCE. Lemma 3.6.6 then implies that $\bar{c}_{i} \geq \eta_{i}$. Suppose first that $\bar{c}_{i}=\eta_{i}$. Since $\eta_{i}$ and $1-\eta_{i}$ are close to each other, player $j$ could obtain the payoff of $1-\eta_{i}$ by committing to it. Thus, we must have that $\bar{c}_{i}>\eta_{i}$. But we have shown before that this is not consistent with SMCE, a contradiction.

Finally, we have to show that any SMCE is efficient. Suppose by way of contradiction that there is some SMCE leading to payoffs $\left(\bar{u}_{1}, \bar{u}_{2}\right)$ such that $\bar{u}_{i} \geq 1-\eta_{j}$ for both $i=1,2$ and $j \neq i$ but $\bar{u}_{1}+\bar{u}_{2}<1$. Let $\left(\bar{c}_{1}, \bar{c}_{2}\right)$ be the commitments in the supposed SMCE. Lemma 3.5.2 implies that if an agreement $x$ is reached in round $t$, then $x_{1}+x_{2}=\delta^{t}$. Since $\bar{u}_{1}+\bar{u}_{2}<1$, the supposed SMCE must involve disagreement at $t=0$. Disagreement continues until time $\tau\left(c_{k}\right)$, where we assume $c_{k} \geq c_{l}$ without loss of generality. By Corollary 3.5.5, we have $\bar{u}_{l} \leq \varphi_{l} \delta^{\tau\left(c_{k}\right)}$. But the supposition is that $\bar{u}_{l} \geq 1-\eta_{k}$. These two inequalities imply that $\eta_{k}+\varphi_{l} \delta^{\tau\left(c_{k}\right)} \geq 1$. But by the definition of $\eta_{k}$, we have $\eta_{k}+\varphi_{l} \delta^{\tau\left(\eta_{k}\right)} \leq 1$. We see that $c_{k} \geq \eta_{k}$. But we have already shown that $c_{k} \leq \eta_{k}$ in an SMCE; thus we can conclude that $c_{k}=\eta_{k}$ and, moreover, $c_{k}+\varphi_{l} \delta^{\tau\left(c_{k}\right)}=1$. By Lemma 3.6.2, player $l$ has two potential best-responses, namely $c_{l}^{\prime}=1-c_{k}$ and $c_{l}^{\prime \prime}=\psi_{l} \delta^{\tau\left(c_{k}\right)}$. In the former case, immediate agreement is reached since $c_{k}=\eta_{k}$ and $1-c_{k}$ are close to each other, a contradiction. Consider the latter case. At round $t=0$, player 2's aspiration will be $\varphi_{l} \delta^{\tau\left(c_{k}\right)}$. But since $\eta_{k}+\varphi_{l} \delta^{\tau\left(\eta_{k}\right)} \leq 1$ and $c_{k}=\eta_{k}$, the aspirations at time $t=0$ sum up to exactly one. But any SMCE strategy profile induces an SPBE when restricted to the bargaining subgame, and in an SPBE agreement is reached when the aspirations sum to the available surplus. Therefore, we find immediate agreement, a contradiction.

Passing to the limit as $\delta \rightarrow 1$, we find the following equilibrium range.

Theorem 3.6.10 If $\delta$ is sufficiently close to one, the surplus division $\left(x_{1}, x_{2}\right) \in \mathbb{R}_{+}^{2}$ can be supported by an SMCE of the simultaneous commitment game if and only if $x_{1}+x_{2}=1$ and $x_{i} \geq \frac{1}{3-\beta_{i}}$ for both $i=1,2$.

One implication of Theorem 3.6.10 is that the division $\left(\beta_{1}, \beta_{2}\right)$ need not be supported by an equilibrium if it is very lopsided towards one player. Put another way, if the distribution of proposal power is very disadvantageous for one player, then the commitment power can mitigate this disadvantage, even if both players have access to the commitment device simultaneously.

With the conventional notion of irrevocable share-commitments, one would expect the simultaneous commitment case to be a mere coordination problem in which any distribution 
can be supported by some equilibrium. In the model at hand, this is still nearly true if $\delta$ is close to zero. However, if $\delta$ is close to one, the range of equilibrium divisions shrinks considerably. More precisely, in the limit as $\delta \rightarrow 1$, the share of the surplus whose allocation is left unpredicted by SMCE is at most one fifth. Conversely, for large $\delta$, SMCE is sufficiently strong as a solution concept to determine eighty percent of the allocation. Moreover, we note that each player's share is bounded below by one third, even with arbitrarily low recognition probability.

Comparing the results for the sequential and simultaneous commitment games, two common points emerge.

First, our model yields predictions tantamount to what one would expect with irrevocable, everlasting commitments if $\delta$ is close to zero, but produces very different results if $\delta$ is close to one. The intuition is that with a small $\delta$, the option to hold out until the opponent's commitment becomes void is very unattractive and hence commitment confers a lot of power.

Second, for large $\delta$, the ability to make a commitment of the type which we propose ensures that a player will get at least one third of the surplus, even if his recognition probability is arbitrarily small. In a sense, the ability to commit is worth one third of the surplus to each player, while the value of proposal power lies in determining the allocation of the remaining third.

Since the range of SMCE divisions depends on the recognition probabilities and the discount factor, we ask whether there is any equilibrium division which is robust to changes in these parameters. The following theorem claims that the equal split of the surplus is consistent with SMCE irrespective of the aforementioned parameters. Moreover, the equal split is the only division with this property.

Theorem 3.6.11 A surplus division $\left(x_{1}, x_{2}\right) \in \mathbb{R}_{+}^{2}$ can be supported by an SMCE of the simultaneous commitment game for all $\delta$ and for all $\beta$ if and only if $\left(x_{1}, x_{2}\right)=\left(\frac{1}{2}, \frac{1}{2}\right)$.

Proof: If: Since $\eta_{i} \geq \frac{1}{2}$ for $i=1,2$, we also have $1-\eta_{i} \leq \frac{1}{2}$. The claim follows from Theorem 3.6.9.

Only if: Consider a pie division in which player $k=1,2$ obtains a payoff of $\frac{1}{2}-\varepsilon$, where $\varepsilon>0$. By Theorem 3.6.10, if $\delta$ is sufficiently large, SMCE requires $\frac{1}{2}-\varepsilon \geq \frac{1}{3-\beta_{k}}$. This can be rewritten as $\beta_{k} \leq \frac{\frac{1}{2}-3 \varepsilon}{\frac{1}{2}-\varepsilon}$. But we have assumed that $\varepsilon>0$, thus $\frac{\frac{1}{2}-3 \varepsilon}{\frac{1}{2}-\varepsilon}<1$. Consequently, choosing $\delta$ sufficiently large and $\beta_{k} \in\left(\frac{\frac{1}{2}-3 \varepsilon}{\frac{1}{2}-\varepsilon}, 1\right)$ ensures that the pie division under consideration is not supported by any SMCE.

In the simultaneous commitment game, any division $(x, 1-x)$ with $0<x<1$ can be supported by SMCE for some choice of $\beta$ and $\delta$, but Theorem 3.6.11 shows that the equal split is unique in being consistent with SMCE for any choice of these parameters. The equal split emerges as a robust focal point within the range of equilibria. 


\subsection{Conclusion}

We have studied the division of a shrinking surplus through a bilateral bargaining procedure with commitment. We have proposed a new notion of commitment, which has two main characteristics. Commitment is stated in time value terms, and a commitment expires when it has been rendered infeasible by the passage of time and the ensuing shrinkage of the surplus. As a result of this modeling approach, there is a trade-off between the amount to which one commits and the duration of the commitment. Relatively moderate commitments stay in effect for longer than extreme commitments.

We have focussed our analysis on three games, namely, a game in which only one player can commit before bargaining starts, a game in which both players commit sequentially before bargaining starts, and a game in which the two players commit simultaneously prior to the bargaining stage. In each of these cases, we have applied slight refinements of subgame-perfect equilibrium as solution concepts. In an equilibrium, we always find immediate agreement on a division which corresponds to the commitments. In a sense, the agreement is already predetermined in the commitment stage, while the bargaining stage plays the role of a threat which has a moderating influence on players' choice of a commitment.

In the game with one committed player, we have uniquely predicted the division of the surplus. The committed player obtains strictly between one half and the entire surplus, and always does strictly better than in a benchmark game without commitment. The equilibrium division of the surplus depends in a discontinuous and non-monotonic way on the discount factor. However, when the discount factor is very small, commitment confers the most bargaining power. Conversely, when the discount factor is very close to one, the recognition probabilities are relatively important as a source of bargaining power. More precisely, if the discount factor is close to one and the recognition probability of the committed player is close to zero, then the surplus is divided nearly equally. In this sense, proposal power and commitment power are "equally important" in the limit.

In the game where both players commit sequentially, we have also found a unique prediction for the division of the surplus. There is a first-mover advantage since the first mover obtains between one half and two thirds of the surplus, depending on the choice of the recognition probabilities.

In the game with simultaneous commitments, a range of surplus divisions is supported by equilibria. If the discount factor is sufficiently large, this range shrinks to at most one fifth of the entire range of feasible divisions. If the commitments were of unlimited duration, then we would expect the strategic situation to collapse to a pure coordination problem, where subgame-perfect equilibrium (or a technical refinement thereof) would have no predictive power with regard to the division. With our notion of commitment, the range of equilibrium divisions is significantly smaller when we choose the discount factor large enough. Moreover, in the model at hand, the equal split emerges as a focal point within the range of equilibrium divisions. We have established that the equal split is the only division which is supported by an equilibrium regardless of the values of the discount factor and the recognition probabilities. 


\section{Theory of the firm - Bargaining and competitive equi- librium}

\subsection{Introduction}

Suppose that a firm has several owners and that the future is uncertain in the sense that one out of many different states of nature will realize tomorrow. An owner's time preference and risk attitude will determine the importance he places on payoffs in the different states. It is a well-known problem in the literature that under incomplete asset markets, a conflict about the firm's objective function tends to arise among its owners.

We take a non-cooperative bargaining approach to this conflict, which is new to the literature. We present a model in which the internal decision making of the firm is formalized explicitly as a strategic bargaining game. In contrast to standard theory, we thus consider the firm as a coalition of owners who use strategic power in order to influence the firm's production decision and thereby maximize their own payoffs. ${ }^{7}$

The standard approach in the existing literature on incomplete markets with production originates from contributions by Diamond (1967), Drèze (1974), and Grossman and Hart (1979). Diamond (1967) formulates the notion of constrained Pareto optimality, an optimality notion that takes the incompleteness of asset markets into account. Drèze (1974) shows that necessary first-order conditions for constrained Pareto optimality require that the firm should choose a production plan which is optimal when evaluated against a weighted average of the shareholders' utility gradients, where the weights are given by the ownership shares. This objective function for the firm is usually referred to as the Drèze criterion.

Standard notions of constrained Pareto optimality allow for arbitrary redistributions in the initial period, and so does Drèze (1974), pp. 141-142:

"The definition does not place any restrictions on the allocation among consumers of the adjustments in current consumption required to offset the adjustment in the input level $a^{j}-\bar{a}^{j}$. Alternatively stated, the definition is consistent with arbitrary transfers of initial resources among consumers."

Arbitrary redistributions in the initial period leads to indeterminateness of equilibrium. One would in general expect a multiplicity of one degree less than the number of shareholders.

Grossman and Hart (1979) use the Drèze criterion as the objective function for the firm, but with final ownership shares replaced by initial ones. Contrary to Drèze (1974), they also require that at equilibrium no transfers are made in the initial period. The advantage of this approach is that it leads to determinateness of equilibrium, its downside is inconsistency. Indeed, initial period transfers are used in Grossman and Hart (1979) in their definition of constrained Pareto optimality, which motivates the decision criterion for the firm. At the same time, the equilibrium decision of the firm is imposed to involve no transfers itself. Absence

\footnotetext{
${ }^{7}$ This chapter corresponds to the paper V.Britz, P.J.J.Herings, and A.Predtetchinski (2010), "Theory of the firm - Bargaining and competitive equilibrium, METEOR Research Memorandum RM/10/05\%.
} 
of initial-period transfers has become the standard concept of equilibrium, called stock market equilibrium, see for instance the treatment in the authoritative book of Magill and Quinzii (1996) and in the paper by Dierker, Dierker, and Grodal (2002) on the constrained inefficiency of stock market equilibria.

Recognizing that the Drèze criterion is normative in nature, some authors have tried to link it to outcomes of majority voting. A typical approach in this stream of literature is to ask: If a certain production plan was given as a default option, would there exist an alternative plan preferred by more than at least a certain (super-)majority of the shareholders? If no winning alternative can be found, the default plan is considered "stable." Tvede and Crès (2005) discuss the relationship between the Drèze criterion and such a voting approach and find conditions under which both lead to compatible predictions. Another voting analysis is given by DeMarzo (1993) who emphasizes the importance of the largest shareholder. Kelsey and Milne (1996) give a proof for equilibrium existence in a more general model which emphasizes externalities between firms and shareholders. However, both stock market equilibria and the approaches related to majority voting seem to suffer from a common problem. Both approaches ask only which production plans are stable to being replaced by other plans through a certain mechanism. However, there is no explanation why a particular plan would serve as a default setting or how any one particular plan is to be chosen in case there are several plans satisfying the criterion used.

Few papers have taken a truly positive approach to decision making within the firm, as is the case in this paper.

In our setup, there is a single firm which exists in an environment of competitive but potentially incomplete markets. We take the ownership structure of the firm as exogenously fixed to focus attention on the decision making within the firm, rather than on the role of expectations regarding future stock price as a consequence of current production decisions and the identity of future shareholders as in Bonnisseau and Lachiri (2004) and Drèze, Lachiri, and Minelli (2009). When applied to our setting without stock markets, we refer to the stock market equilibrium concept as competitive equilibrium.

The firm will be active in two time periods. A production plan and a transfer scheme have to be chosen knowing the state of the world in the first period, but under uncertainty about the state of the world in the second period. There are assets which the owners can use to shift consumption across time periods and states. Owners are price-takers in the asset markets.

We address the issue raised in Magill and Quinzii (1996), who write on p. 364 when referring to the concept of partnership equilibrium:

"A weakness of this concept of equilibrium is that it does not provide a well-defined bargaining process by which partners could come up with such an agreement."

In this paper, we propose such a bargaining process. We have the owners of the firm bargain in order to determine a production plan for the firm. In each round of bargaining, an owner is chosen to make a proposal. This owner offers a production plan as well as a transfer scheme of side-payments in terms of first-period consumption. If the owners unanimously agree, the proposal is implemented. Otherwise, the negotiation breaks down with some probability, in 
which case no production takes place and no transfers are made. With the complementary continuation probability, bargaining continues to another bargaining round. In the case of perpetual disagreement, the firm will not produce, and no transfers will be made. Through the bargaining process, the owners of the firm collectively decide on a production plan and a transfer scheme. Moreover, as individuals they may invest in the asset markets to determine their amounts of consumption at each state of the world. We refer to our notion of equilibrium as bargaining equilibrium.

Our main results are the following. We show that the bargaining equilibrium corresponds to a weighted Nash Bargaining Solution; a unique prediction for a production plan as well as a system of transfer payments in terms of good 0 is derived. In the special case where markets are complete, the bargaining equilibrium selects the profit-maximizing production plan and is therefore in line with the predictions of the Arrow-Debreu model as far as the firm's production decision is concerned. However, contrary to the Arrow-Debreu model, owners make use of their bargaining power to redistribute the profits among themselves via the transfer scheme. Hence, owners obtain payoffs which are generically different from those in standard economic theory, even if markets are complete.

In the case of incomplete markets, we find that the production plan which the firm adopts under the bargaining equilibrium is almost always different from the competitive equilibrium one. Moreover, non-zero transfers are made in general. At the same time, bargaining equilibria are constrained Pareto optimal, and are therefore consistent with the use of the Drèze criterion. However, since contrary to competitive equilibrium transfers are made, the shareholders' utility gradients are not the same as the competitive equilibrium ones, explaining why the chosen production plan does typically not coincide with the one corresponding to a competitive equilibrium. The bargaining equilibrium satisfies the requirements of an equilibrium as defined in Drèze (1974). Like the competitive equilibrium, the bargaining equilibrium can therefore be viewed as selecting a particular Drèze equilibrium.

In Section 2, we present the model and the most important assumptions formally. In Section 3, we show how bargaining on the production plan and a transfer scheme can be reformulated as bargaining on the implied payoffs. In Section 4, we characterize the relevant bargaining outcome. In Section 5, this bargaining outcome is interpreted in light of the existing literature and, in particular, compared to the Drèze criterion. Section 6 concludes.

\subsection{Model Description}

We study a firm with several owners in a setting with incomplete markets. Each owner $i=$ $1, \ldots, I$ holds a share $\theta^{i}>0$ of the firm so that $\sum_{i=1}^{I} \theta^{i}=1$. The set of owners $\{1, \ldots, I\}$ will be denoted by $\mathcal{I}$.

The firm carries out some productive activity which extends over two periods. In the first period, the state of the world is known to be $s=0$. In the second period, any one of the states of the world $s=1, \ldots, S$ may be realized; we write $\mathcal{S}=\{0,1, \ldots, S\}$. A particular productive activity of the firm is described by a production plan $y \in \mathbb{R}^{S+1}$. If $y_{s}<0$ for some $s \in \mathcal{S}$, then each owner $i$ has to provide the firm with an input of $\left|\theta^{i} y_{s}\right|$ in state $s$. Similarly, if $y_{s}>0$, then 
this output will be distributed to the owners in the proportion in which they own the firm. The set of all production plans which are feasible for the firm is called its production set and is denoted by $Y$.

Each owner $i$ has initial endowments $\omega^{i} \in \mathbb{R}_{++}^{S+1}$, which can be used to finance the provision of inputs. Consumption in state $s=0$ can be transferred across owners and assets can be used to shift consumption across states. Transfers and assets will be introduced in detail in the sequel.

A production plan can be chosen only by unanimous consent of the owners. In order to reach agreement, the following bargaining procedure is used. Bargaining takes place in a, potentially infinite, number of rounds $r=0,1, \ldots$. In the beginning of any round $r$, a draw from a given probability distribution $\mu \in \Delta^{I}$, where $\Delta^{I}$ is the set of strictly positive vectors in the unit simplex in $\mathbb{R}^{I}$, determines the proposer in round $r .^{8}$

This proposer then makes an offer $(y, t) \in Y \times T$, where $T=\left\{t \in \mathbb{R}^{I} \mid \sum_{i \in \mathcal{I}} t^{i} \leq 0\right\}$. Here $t^{i}$ denotes the transfer owner $i$ receives, and which is made in terms of consumption in state 0 . The owners then either accept or reject the offer in some given order. It is assumed that an owner cannot accept a proposal which leads to his insolvency irrespective of his choice of an asset portfolio. This assumption will be stated more formally later on.

If unanimous agreement ${ }^{9}$ on the production plan and transfer scheme is reached, the bargaining stage ends and the chosen production plan and transfer scheme are implemented. If, however, an owner rejects a proposal, bargaining moves to round $r+1$ with probability $\delta$. With probability $1-\delta$, a breakdown of bargaining occurs. In that case, we assume that no production will take place and no transfers are made. Each individual owner is merely left to choose his asset portfolio. Likewise, perpetual disagreement means that no production takes place. One interpretation of the breakdown probability is that the investment opportunities implicit in the production set may slip away if one waits too long to exploit them.

Once bargaining has led to an agreement (or broken down), the sequel of the model does not incorporate any strategic interaction anymore. Each owner individually decides on an asset portfolio. The owner can purchase assets $j=1, \ldots, J$ at exogenously given prices $q_{1}, \ldots, q_{J}$. Owners act as price takers in the asset markets. In state $s=1, \ldots, S$, each unit of asset $j$ will give a payoff of $a_{s}^{j}$. We summarize the asset structure in the $(S \times J)$-matrix $A$, which we assume to be of full column rank. Redundant assets are ignored without loss of generality. It

\footnotetext{
${ }^{8}$ We do not place further restrictions on the distribution of bargaining power. In particular, bargaining power may but need not be determined by the shares of ownership. The model can accommodate the case where, for example, everybody has equal bargaining power, but also the case where bargaining power does increase with the share of ownership. Bargaining power may therefore capture strengths (such as bargaining skill) which are not derived from the firm's ownership structure.

${ }^{9} \mathrm{We}$ mainly have in mind rather small firms (such as partnerships) where the unanimity rule is a realistic assumption at least for major decisions. Considering decision-making among the shareholders of a corporation, one can easily extend the model to a more general approval rule (such as majority voting). However, this would greatly complicate the equilibrium analysis. In particular, we expect that it would require considering equilibria in mixed strategies.
} 
will sometimes be convenient to use the notation

$$
W=\left(\begin{array}{c}
-q \\
A
\end{array}\right) \text {. }
$$

Assets are perfectly divisible and may be sold short. We write $z_{j}^{i}$ for owner $i$ 's holdings of asset $j$.

Economic activity in state 0 therefore consists of the implementation of the agreed production plan and transfers, the choice of asset portfolios, and consumption. Next, one state of nature $s \in\{1, \ldots, S\}$ realizes, and contingent on $s$ the assets pay off, the firm realizes its output, and the owners consume.

Owner $i$ has preferences over consumption plans $x^{i} \in \mathbb{R}_{++}^{S+1}$, which are represented by a von Neumann-Morgenstern utility function $u^{i}: \mathbb{R}_{++}^{S+1} \rightarrow \mathbb{R}$. Given the bargaining outcome $(y, t) \in Y \times T$, owner $i$ solves the following maximization problem:

$$
\begin{aligned}
\max _{x^{i} \in \mathbb{R}_{++}^{S+1}, z^{i} \in \mathbb{R}^{J}} u^{i}\left(x^{i}\right) \text { subject to } x_{0}^{i} & =\omega_{0}^{i}+\theta^{i} y_{0}+t^{i}-q z^{i}, \\
x_{s}^{i} & =\omega_{s}^{i}+\theta^{i} y_{s}+A_{s} z^{i}, s=1, \ldots, S .
\end{aligned}
$$

Let $e(0)$ denote the $(S+1)$-dimensional column vector $(1,0, \ldots, 0)^{\top}$. For $i \in \mathcal{I}$, we define

$$
B^{i}=\left\{(y, t) \in Y \times T \mid \exists z^{i} \in \mathbb{R}^{J}, \omega^{i}+\theta^{i} y+e(0) t^{i}+W z^{i} \gg 0\right\},
$$

and $B=\cap_{i \in \mathcal{I}} B^{i}$. The set $B$ contains all bargaining outcomes which allow each owner to remain solvent by an appropriate portfolio choice. Solvency in this context means a strictly positive consumption in each state. Owner $i$ can only accept a proposal if it belongs to $B^{i}$. Since $\omega^{i} \gg 0$ for all $i \in \mathcal{I}$, it holds that $(0,0) \in B$ and thus $B$ is non-empty.

The results in this paper are derived under a number of assumptions on the utility functions, the production possibility set, and the asset structure. These assumptions are now introduced.

\section{Assumption 4.2.1 (Production Set)}

1. $Y$ is closed, strictly convex, and bounded from above.

2. $Y \supset \mathbb{R}_{-}^{S+1}$ : Output can be freely disposed of and inaction is possible.

3. $Y \cap \mathbb{R}_{+}^{S+1} \subset\{0\}$ : One cannot produce a positive output without inputs.

4. $\partial Y$ is a $C^{2}$ manifold with nonzero Gaussian curvature.

\section{Assumption 4.2.2 (Utility functions)}

For all $i \in \mathcal{I}$ we assume the following.

1. $u^{i}$ is twice continuously differentiable on $\mathbb{R}_{++}^{S+1}$.

2. $u^{i}$ is differentiably strictly concave on $\mathbb{R}_{++}^{S+1}$, i.e. the Hessian matrix of $u^{i}$ is negative definite on $\mathbb{R}_{++}^{S+1}$. 
3. For any $s=0,1, \ldots, S$, and any $x_{-s}^{i} \in \mathbb{R}_{++}^{S}$ it holds that $u^{i}\left(x_{s}^{i}, x_{-s}^{i}\right)$ goes to negative infinity in the limit as $x_{s}^{i}$ approaches zero.

We define the set of normalized state prices $\Pi$ by

$$
\Pi=\left\{\pi \in \mathbb{R}_{++}^{S} \mid(1, \pi) W=0\right\}
$$

Asset markets are said to be complete if $\Pi$ is single-valued. If $\Pi$ is not single-valued, markets are called incomplete. We also note that $\Pi$ is a convex set.

\section{Assumption 4.2.3 (Asset Structure)}

1. The matrix $A$ has full column-rank.

2. The set $\Pi$ is non-empty.

3. There is $\pi \in \Pi$ such that $\pi$ is not normal to $Y$ at $y=0$.

Assumption 2.3.2 would be satisfied in general equilibrium, but has to be imposed here since we conduct our analysis in a partial equilibrium context. Assumption 2.3.3 rules out the (uninteresting) special case in which markets are complete and the unique profit-maximizing production plan is $y=0$.

\subsection{Reduced Form Bargaining Game}

When the owners bargain about a production plan and a transfer scheme, they implicitly bargain about the associated payoffs. In this section, we will analyze the bargaining problem in payoff space. In order to motivate this approach, we will show that, given efficiency and individual rationality, there is a one-to-one correspondence between both problems. In other words, any Pareto-efficient and individually rational outcome of the bargaining problem in the payoff space is supported by a unique combination of a production plan, a transfer scheme, and asset portfolios for each owner. This result is driven by the assumptions of strict convexity of the production set and strict concavity of the utility functions.

Consider some $(y, t) \in B$. By definition of $B$, there must be some $z \in \mathbb{R}^{I J}$ such that the vector $x^{i}=\omega^{i}+\theta^{i} y+e(0) t^{i}+W z^{i}$ is strictly positive for all $i \in \mathcal{I}$. Using Assumption 2.3, we see that the set of portfolio choices for owner $i$ which lead to a utility of at least $u^{i}\left(x^{i}\right)$ is compact. Hence, the indirect utility $\bar{u}^{i}(y, t)$ is well-defined. We write $\bar{u}(y, t)$ for the profile of utilities $\left(\bar{u}^{1}(y, t), \ldots, \bar{u}^{I}(y, t)\right)$. It holds that the optimal consumption bundle, denoted by $\xi^{i}(y, t)$, is unique. Suppose to the contrary that there are two distinct feasible consumption bundles $x^{i}$ and $\bar{x}^{i}$ leading to utility $\bar{u}^{i}(y, t)$ Since any convex combination of $x^{i}$ and $\bar{x}^{i}$ is feasible and gives strictly higher utility than $\bar{u}^{i}(y, t)$, we have a contradiction. The uniqueness of $\xi^{i}(y, t)$ combined with the full column-rank of $A$ implies that the optimal portfolio choice is unique. The set $V$ of feasible payoffs for the owners is given by $V=\bar{u}(B)$, a subset of $\mathbb{R}^{I}$.

We denote by $V^{+}$the individually rational part of $V$, and by $\partial V$ and $\partial V^{+}$the weak Pareto boundaries of the sets $V$ and $V^{+}$. Individually rational payoffs are here defined as the payoffs 
that exceed the payoffs $v^{0}=\bar{u}(0,0)$ owners could achieve without relying on firm production and transfers, but by making use of trades in the asset market. We define

$$
\begin{aligned}
V^{+} & =\left\{v \in V \mid v \geq v^{0}\right\}, \\
\partial V & =\left\{v \in V \mid \nexists v^{\prime} \in V, v^{\prime} \gg v\right\}, \\
\partial V^{+} & =V^{+} \cap \partial V .
\end{aligned}
$$

The next five lemmas state that the set $V^{+}$satisfies a number of desirable properties. In particular, we demonstrate that $V^{+}$is compact and convex, that $V$ is comprehensive from below, that all points in $\partial V^{+}$are strongly Pareto efficient, and that there is a unique vector in the normal to $V$ at any point in $\partial V^{+}$.

Lemma 4.3.1 The set $V$ is comprehensive from below.

Proof: Take $\bar{v} \in V$. We want to show that any $v \in \mathbb{R}^{I}$ such that $v \leq \bar{v}$ belongs to $V$. Let $(\bar{x}, \bar{y}, \bar{t}, \bar{z}) \in \mathbb{R}_{++}^{(S+1) I} \times B \times \mathbb{R}^{J I}$ be such that $\bar{v}=\bar{u}(\bar{y}, \bar{t})$ and let $(\bar{x}, \bar{z})$ correspond to optimal consumption choices and portfolio plans given $(\bar{y}, \bar{t})$. Consider a particular $i \in \mathcal{I}$, and define the set $L^{i}$ as follows.

$$
L^{i}=\left\{x^{i} \in \mathbb{R}_{+}^{S+1} \mid \exists z^{i} \in \mathbb{R}^{J}, x^{i}=\omega^{i}+\theta^{i} \bar{y}+e(0) \bar{t}^{i}+W z^{i}\right\} .
$$

The set $L^{i}$ is non-empty, closed, and bounded. Thus, there exists $\hat{x}^{i} \in L^{i}$ such that $\hat{x}_{0}^{i} \geq x_{0}^{i}$ for all $x^{i} \in L^{i}$. Let $\hat{z}^{i}$ be the asset portfolio associated with $\hat{x}^{i}$.

For some $\kappa \in(0,1)$, define $\tilde{x}^{i}=\kappa \bar{x}^{i}+(1-\kappa)\left(0, \hat{x}_{1}^{i}, \ldots, \hat{x}_{S}^{i}\right)^{\top}$. The consumption plan $\tilde{x}^{i}$ results from the production plan $\bar{y}$, the transfer $\tilde{t}^{i}:=\bar{t}^{i}-(1-\kappa) \hat{x}_{0}^{i}$, and the asset portfolio $\tilde{z}^{i}=\kappa \bar{z}^{i}+(1-\kappa) \hat{z}^{i}$.

Since $\kappa$ is strictly positive by construction, we have that $\tilde{x}^{i} \in \mathbb{R}_{++}^{S+1}$, and therefore $(\bar{y}, \tilde{t}) \in B^{i}$.

By construction of $\hat{x}^{i}$ and $\tilde{t}^{i}$, it holds that $\xi_{0}^{i}(\bar{y}, \tilde{t}) \leq \hat{x}_{0}^{i}-\bar{t}^{i}+\tilde{t}^{i}=\kappa \hat{x}_{0}^{i}$.

We can use a direct argument to show that $\xi^{i}$ is continuous in $(y, t) \in B^{i}$, and we have assumed that the direct utility function $u^{i}$ is continuous on $\mathbb{R}_{++}^{S+1}$. Thus, the indirect utility function $\bar{u}^{i}=u^{i} \circ \xi^{i}$ is continuous and reaches any value in the interval $\left[\bar{u}^{i}(\bar{y}, \tilde{t}), \bar{u}^{i}(\bar{y}, \bar{t})\right]$. The statement follows from passing to the limit as $\kappa \downarrow 0$, in which case $\xi_{0}^{i}(\bar{y}, \tilde{t})$ becomes arbitrarily small and Assumption 2.2.3 implies that $\bar{u}^{i}(\bar{y}, \tilde{t})$ becomes arbitrarily negative. Finally, notice that our construction for owner $i$ only involves the transfer $t^{i}$, so that we can deal with the functions $\bar{u}^{1}, \ldots, \bar{u}^{I}$ independently.

We have defined $\partial V^{+}$as the weak Pareto-boundary of $V^{+}$. We will show next that it coincides with the strong Pareto-boundary. That is, $\bar{v}$ belongs to $\partial V^{+}$if and only if there is no $v \in V$ such that $v \geq \bar{v}$, with strict inequality in at least one component.

Lemma 4.3.2 All points in $\partial V^{+}$are strongly Pareto-efficient.

Proof: Consider some $v \in \partial V^{+}$. Let $(x, y, t, z) \in \mathbb{R}_{++}^{(S+1) I} \times B \times \mathbb{R}^{J I}$ be such that $v=\bar{u}(y, t)$ and let $(x, z)$ correspond to an optimal consumption choice and portfolio plan given $(y, t)$. 
Suppose that there is $\bar{v} \in V$ such that $\bar{v}^{i^{\prime}}>v^{i^{\prime}}$ for some $i^{\prime} \in \mathcal{I}$ and $\bar{v}^{i} \geq v^{i}$ for all $i \in \mathcal{I} \backslash\left\{i^{\prime}\right\}$. Let $(\bar{x}, \bar{y}, \bar{t}, \bar{z}) \in \mathbb{R}_{++}^{(S+1) I} \times B \times \mathbb{R}^{J I}$ be such that $\bar{v}=\bar{u}(\bar{y}, \bar{t})$ and let $(\bar{x}, \bar{z})$ correspond to an optimal consumption choice and portfolio plan given $(\bar{y}, \bar{t})$. For $\varepsilon \in\left(0, \bar{x}_{0}^{i^{\prime}}\right)$, we construct the consumption plan $\tilde{x}^{i^{\prime}}=\bar{x}^{i^{\prime}}-\varepsilon e(0)$ and $\tilde{x}^{i}=\bar{x}^{i}+(\varepsilon /(I-1)) e(0)$ for $i \in \mathcal{I} \backslash\left\{i^{\prime}\right\}$. Since $u^{i^{\prime}}$ is continuous, $u^{i^{\prime}}\left(\tilde{x}^{i^{\prime}}\right)>u^{i^{\prime}}\left(x^{i^{\prime}}\right)$ for $\varepsilon$ sufficiently small. Assumption 2.2.2 implies that $u^{i}\left(\tilde{x}^{i}\right)>u^{i}\left(x^{i}\right)$ for all $i \in \mathcal{I} \backslash\left\{i^{\prime}\right\}$. We define the transfer scheme $\tilde{t} \in T$ by $\tilde{t}^{i^{\prime}}=\bar{t}^{i^{\prime}}-\varepsilon e(0)$ and $\tilde{t}^{i}=\bar{t}^{i}+(\varepsilon /(I-1)) e(0)$ for $i \in \mathcal{I} \backslash\left\{i^{\prime}\right\}$. Then $\bar{u}(\bar{y}, \tilde{t}) \geq\left(u^{1}\left(\tilde{x}^{1}\right), \ldots, u^{I}\left(\tilde{x}^{I}\right)\right) \gg v$, a contradiction to the weak Pareto efficiency of $v$.

Lemma 4.3.3 The set $V^{+}$is compact.

\section{Proof:}

We define the set $X$ as follows,

$$
X=\left\{x \in \mathbb{R}_{+}^{(S+1) I} \mid \exists(y, t, z) \in B \times \mathbb{R}^{J I}, \forall i \in \mathcal{I}, x^{i}=\omega^{i}+\theta^{i} y+e(0) t^{i}+W z^{i}\right\} .
$$

We will show first that $X$ is bounded.

Since $X$ is a subset of $\mathbb{R}_{+}^{(S+1) I}$, it is clearly bounded from below. To show it is bounded from above, take any $x \in X$ and compute

$$
\sum_{i \in \mathcal{I}} x^{i}=\sum_{i \in \mathcal{I}} \omega^{i}+y+e(0) \sum_{i \in \mathcal{I}} t^{i}+W \sum_{i \in \mathcal{I}} z^{i} \leq \sum_{i \in \mathcal{I}} \omega^{i}+y+W \sum_{i \in \mathcal{I}} z^{i} .
$$

By Assumption 2.3.2, the set $\Pi$ is non-empty. For $\pi \in \Pi$ it holds that $\pi \in \mathbb{R}_{++}^{S}$ and $(1, \pi) W=0$, so

$$
(1, \pi) \sum_{i \in \mathcal{I}} x^{i} \leq(1, \pi)\left(\sum_{i \in \mathcal{I}} \omega^{i}+y\right) .
$$

It follows that $(1, \pi) \sum_{i \in \mathcal{I}} x^{i}$ is bounded from above since $Y$ is bounded from above by Assumption 2.1.1. Since $x^{i}$ is bounded from below for all $i,(1, \pi) \sum_{i \in \mathcal{I}} x^{i}$ bounded from above implies that $x^{i}$ is bounded from above for all $i \in \mathcal{I}$. We have shown that $X$ is bounded.

The boundedness of $X$ and Assumption 2.2.3 imply that for each $i \in \mathcal{I}$ there is a vector $b^{i} \in \mathbb{R}_{++}^{S+1}$ such that $x \in X$ and $u^{i}\left(x^{i}\right) \geq v^{0 i}$ imply $x^{i} \geq b^{i}$.

Now define the set

$$
X^{*}=X \cap\left\{x \in \mathbb{R}_{+}^{(S+1) I} \mid x^{i} \geq b^{i}, \forall i \in \mathcal{I}\right\}
$$

Since $X^{*} \subset X$, it is immediate that $X^{*}$ is bounded. We will show next that $X^{*}$ is closed. To this end, we define the sets

$$
\begin{aligned}
\tilde{Y} & =\left\{\left(\theta^{1} y, \ldots, \theta^{I} y\right) \in \mathbb{R}^{(S+1) I} \mid y \in Y\right\} \\
\tilde{T} & =\left\{\tau \in \mathbb{R}^{(S+1) I} \mid \sum_{i \in \mathcal{I}} \tau_{0}^{i} \leq 0 ; \forall i \in \mathcal{I}, \forall s \neq 0, \tau_{s}^{i}=0\right\}, \\
\tilde{W} & =\prod_{i \in \mathcal{I}} \mathcal{R}(W),
\end{aligned}
$$

where $\mathcal{R}(W)$ denotes the column space of $W$. We can write $X^{*}$ as the intersection of the closed set $\left\{x \in \mathbb{R}_{+}^{(S+1) I} \mid x^{i} \geq b^{i}, \forall i \in \mathcal{I}\right\}$ and the set $\{\omega\}+\tilde{Y}+\tilde{T}+\tilde{W}$. We show the latter set to be closed, thereby proving that $X^{*}$ is closed. 
To show that $\{\omega\}+\tilde{Y}+\tilde{T}+\tilde{W}$ is closed, we show that the asymptotic cones of the terms in the sum are positively semi-independent. Since $\{\omega\}$ is bounded, the asymptotic cone $\mathrm{A}(\{\omega\})=\{0\}$. Since $\tilde{Y}$ is bounded from above, $\mathrm{A}(\tilde{Y})$ is contained in $-\mathbb{R}_{+}^{(S+1) I}$. Since $\tilde{T}$ and $\tilde{W}$ are cones themselves, they coincide with their asymptotic cones.

Let $y, \tau$, and $w$ be elements of $\mathrm{A}(\tilde{Y}), \mathrm{A}(\tilde{T})$, and $\mathrm{A}(\tilde{W})$ summing up to zero. We show that $y, \tau$, and $w$ are zero vectors, thereby proving that the asymptotic cones are positively semi-independent. For $i \in \mathcal{I}$, let $z^{i} \in \mathbb{R}^{J}$ be such that $w^{i}=W z^{i}$. We have that

$$
\begin{array}{ll}
y_{0}^{i}+\tau_{0}^{i}-q z^{i}=0, & i \in \mathcal{I}, \\
y_{-0}^{i}+A z^{i}=0, & i \in \mathcal{I} .
\end{array}
$$

We take a weighted sum of these equalities, with weights equal to $(1, \pi)$ for some $\pi \in \Pi$ and obtain

$$
y_{0}^{i}+\tau_{0}^{i}-q z^{i}+\pi y_{-0}^{i}+q z^{i}=0, \quad i \in \mathcal{I} .
$$

Finally, we take the sum over $i \in \mathcal{I}$ and find

$$
\sum_{i \in \mathcal{I}} y_{0}^{i}+\sum_{i \in \mathcal{I}} \tau_{0}^{i}+\pi \sum_{i \in \mathcal{I}} y_{-0}^{i}=0
$$

Since the vector $\pi$ is strictly positive, $\sum_{i \in \mathcal{I}} \tau_{0}^{i} \leq 0$, and $y^{i} \in-\mathbb{R}_{+}^{S+1}$, we find that $y^{i}=0$ for all $i \in \mathcal{I}$ and $\sum_{i \in \mathcal{I}} \tau_{0}^{i}=0$. For all $i \in \mathcal{I}$, since $0=y_{-0}^{i}=-A z^{i}$, the full column-rank of $A$ implies that $z^{i}=0$, and consequently that $w^{i}=0$. Now it holds that $\tau_{0}^{i}=-y_{0}^{i}-w_{0}^{i}=0$. We have shown that the set $X^{*}$ is closed.

We have assumed the utility functions to be continuous on the strictly positive orthant. By definition, $X^{*} \subset \mathbb{R}_{++}^{(S+1) I}$, and so the utility functions are continuous on $X^{*}$, which we have just shown to be a compact set. Hence, the set $U=\left\{v \in \mathbb{R}^{I} \mid \exists x \in X^{*}, v=\left(u^{1}\left(x^{1}\right), \ldots, u^{n}\left(x^{n}\right)\right)\right\}$ is compact as well. We argue next that $V^{+}$coincides with $U \cap\left\{v \in \mathbb{R}^{I} \mid v \geq v^{0}\right\}$, so is the intersection of a compact set and a closed set, and therefore compact, proving the result. It follows from the definition of the vectors $b^{1}, \ldots, b^{I}$ that $V^{+} \subset U \cap\left\{v \in \mathbb{R}^{I} \mid v \geq v^{0}\right\}$.

Consider some $v \in U \cap\left\{v \in \mathbb{R}^{I} \mid v \geq v^{0}\right\}$. Let $(x, y, t, z) \in X^{*} \times B \times \mathbb{R}^{J I}$ be such that, for all $i \in \mathcal{I}, x^{i}=\omega^{i}+\theta^{i} y+e(0) t^{i}+W z^{i}$, and $u^{i}\left(x^{i}\right)=v^{i}$. Since $\bar{u}(y, t) \geq v \geq v^{0}$ and since $V$ is comprehensive from below by Lemma 3.1, we have that $v \in V^{+}$.

Lemma 4.3.4 The set $V^{+}$is convex.

Proof: Let $v, \bar{v}$ be distinct elements of $V^{+}$, and $\tilde{v}=\alpha v+(1-\alpha) \bar{v}$ for some $\alpha \in(0,1)$.

There are $(y, t),(\bar{y}, \bar{t}) \in B$ such that $\bar{u}(y, t)=v$ and $\bar{u}(\bar{y}, \bar{t})=\bar{v}$. Let $z$ and $\bar{z}$ be such that for all $i \in \mathcal{I}, \xi^{i}(y, t)=\omega^{i}+\theta^{i} y+e(0) t^{i}+W z^{i}$, and $\xi^{i}(\bar{y}, \bar{t})=\omega^{i}+\theta^{i} \bar{y}+e(0) \bar{t}^{i}+W \bar{z}^{i}$.

We define $(\tilde{y}, \tilde{t})=\alpha(y, t)+(1-\alpha)(\bar{y}, \bar{t})$ and, for $i \in \mathcal{I}, \tilde{x}^{i}=\alpha \xi^{i}(y, t)+(1-\alpha) \xi^{i}(\bar{y}, \bar{t})$. Since $B$ is convex, we have that $(\tilde{y}, \tilde{t}) \in B$. Furthermore, since

$$
\tilde{x}^{i}=\omega^{i}+\theta^{i} \tilde{y}+e(0) \tilde{t}^{i}+W\left(\alpha z^{i}+(1-\alpha) \bar{z}^{i}\right),
$$


the consumption plan $\tilde{x}^{i}$ is attainable for $i$. Therefore, it holds that $\bar{u}^{i}(\tilde{y}, \tilde{t}) \geq u^{i}\left(\tilde{x}^{i}\right) \geq \tilde{v}^{i}$ for all $i \in \mathcal{I}$, where the last inequality follows from the concavity of $u^{i}$. Since $V$ is comprehensive from below, we have $\tilde{v} \in V$. Since $v \geq v^{0}$ and $\bar{v} \geq v^{0}$, it follows that $\tilde{v} \in V^{+}$.

The normal of a convex subset $C$ of $\mathbb{R}^{m}$ at a point $\bar{c}$ in $C$ is defined as the set of vectors $c^{*} \in \mathbb{R}^{m}$ satisfying $\left\|c^{*}\right\|=1$ and $(c-\bar{c}) \cdot c^{*} \leq 0$ for every $c \in C$. Equivalent to the uniqueness of the normal at every point in the boundary $\partial C$, we may assume that $\partial C$ is a $C^{1}$ manifold; see Rockafellar (1970).

Lemma 4.3.5 At any point of $\partial V^{+}$there is a unique vector in the normal to $V$.

Proof: Take any $\bar{v} \in \partial V^{+}$. Let $(\bar{y}, \bar{t})$ be such that $\bar{v}=\bar{u}(\bar{y}, \bar{t})$ and define $\bar{x}^{i}=\xi^{i}(\bar{y}, \bar{t}), i \in \mathcal{I}$. We define the set $T^{\prime}$ by

$$
T^{\prime}=\left\{\left(\tau^{1}, \ldots, \tau^{I-1}, \tau^{I}\right) \in \mathbb{R}^{I-1} \times \mathbb{R}_{+} \mid \bar{x}_{0}^{I}-\sum_{i \in \mathcal{I}} \tau^{i}>0 ; \forall i \in \mathcal{I} \backslash\{I\}, \bar{x}_{0}^{i}+\tau^{i}>0\right\}
$$

The interpretation of $\tau \in T^{\prime}$ is that owner $i=1, \ldots, I-1$ receives a transfer $\tau^{i}$ in period 0 additional to $\bar{x}_{0}^{i}$, whereas owner $I$ receives an additional transfer $-\sum_{i \in \mathcal{I}} \tau^{i}$. Notice that $\tau^{I}$ corresponds to an amount of resource that is wasted. We define the function $f: T^{\prime} \rightarrow \mathbb{R}^{(S+1) I}$ by $f_{0}^{I}(\tau)=\bar{x}_{0}^{I}-\sum_{i \in \mathcal{I}} \tau^{i}, f_{0}^{i}(\tau)=\bar{x}_{0}^{i}+\tau^{i}, i \in \mathcal{I} \backslash\{I\}$, and $f_{s}^{i}(\tau)=\bar{x}_{s}^{i}, i \in \mathcal{I}, s \in \mathcal{S} \backslash\{0\}$. We define $K(\bar{v})$ as the image of $T^{\prime}$ under the function $\phi=\left(u^{1} \circ f^{1}, \ldots, u^{I} \circ f^{I}\right)$. Then $\phi^{-1}$ serves as a $C^{2}$ coordinate system for $K(\bar{v})$ around $\bar{v}$, i.e. $\phi^{-1}$ is injective and surjective, $\phi$ is twice differentiable, and we can show that $\phi^{-1}$ is twice differentiable by means of the inverse function theorem using the property that for all $i \in \mathcal{I}, \partial_{x_{0}^{i}} u^{i}\left(\bar{x}^{i}\right)>0$. It follows that $K(\bar{v})$ is a $C^{2}$ manifold with boundary. Since $K(\bar{v})$ is convex, it has a unique outward normal vector at $\bar{v}$, say $v^{*}$.

We want to show that $v^{*}$ is also the unique normal to $V$ at $\bar{v}$. Suppose to the contrary that there is a normal vector $v^{\prime} \neq v^{*}$ to $V$ at $\bar{v}$. Since $v^{\prime}$ cannot be normal to $K(\bar{v})$, there is $v \in K(\bar{v})$ such that $(v-\bar{v}) \cdot v^{\prime}>0$. But $K(\bar{v}) \subset V$, so that $v \in V$ as well, contradicting that $v^{\prime}$ is normal to $V$ at $\bar{v}$.

It may be interesting to note that the proof of differentiability of $\partial V$ does not rely on the differentiability of $\partial Y$, though we have assumed the latter for later purposes.

We have established that the set $V^{+}$is compact and convex, that the set $V$ is comprehensive from below, that all points in $\partial V^{+}$are strongly Pareto-efficient, and there is a unique vector in the normal to $V$ at any point in $\partial V^{+}$. In the non-cooperative bargaining literature, one considers abstract sets of feasible payoffs which are assumed to have these properties. Therefore, our preceding analysis of the set $V^{+}$demonstrates that the model we study in this paper lends itself to the application of results already established in the bargaining literature. In particular, given an $I$-player bargaining protocol of the type which we have in our model, and a set of feasible payoffs with the aforementioned properties, a characterization of subgame-perfect equilibria in stationary strategies is known in the literature. All such equilibria lead to the selection of payoffs in $\partial V^{+}$and are characterized by absence of delay in reaching an agreement. 
Moreover, it is known that in the limit as $\delta$ goes to one, the payoffs implied by such equilibria converge to a weighted Nash Bargaining Solution, where the weights are given by the vector $\mu$ of recognition probabilities, see Hart and Mas-Colell (1996), Miyakawa (2008), and Laruelle and Valenciano (2007). Britz, Herings, and Predtetchinski (2010) have shown that the result can be suitably generalized to the case where proposers are chosen according to an irreducible Markov process. In this case, the weights of the Nash Bargaining Solution are given by the stationary distribution of this Markov process.

Below, we first give a definition of the weighted Nash Bargaining Solution, and then state the aforementioned convergence result formally as Theorem 4.3.7.

Definition 4.3.6 The $\mu$-weighted Nash Bargaining Solution ( $\mu$-NBS) is the payoff allocation $v^{*} \in V^{+}$which solves

$$
\max _{v \in V^{+}} \prod_{i=1}^{I}\left(v^{i}-v^{0 i}\right)^{\mu^{i}}
$$

The $\mu$-weighted Nash Bargaining Solution can be interpreted as the choice of a social planner who has a Cobb-Douglas social welfare function with weights $\mu$ and set of feasible alternatives given by $V^{+}$. The convexity of $V^{+}$as demonstrated in Lemma 4.3 .4 implies that the $\mu$-weighted Nash bargaining solution is unique.

Theorem 4.3.7 In the limit as $\delta \uparrow 1$, the payoffs of all subgame perfect bargaining equilibria in stationary strategies converge to the $\mu-N B S$.

We finally argue that it is irrelevant whether negotiations are on the implied payoffs directly or on a production plan and transfers since they are in a one to one relationship between each other.

Theorem 4.3.8 For every $\bar{v} \in \partial V^{+}$there is a unique $(\bar{y}, \bar{t}) \in B$ such that $\bar{v}=\bar{u}(\bar{y}, \bar{t})$.

Proof: Consider a payoff vector $\bar{v} \in \partial V^{+}$and $(y, t),(\bar{y}, \bar{t}) \in B$ such that $\bar{u}(y, t)=\bar{u}(\bar{y}, \bar{t})=$ $\bar{v}$. We want to show that $(y, t)=(\bar{y}, \bar{t})$. We have that $\xi^{i}(y, t) \gg 0$ and $\xi^{i}(\bar{y}, \bar{t}) \gg 0$ for all $i \in \mathcal{I}$.

Suppose that there exists some $i^{\prime} \in \mathcal{I}$ such that $\xi^{i^{\prime}}(y, t) \neq \xi^{i^{\prime}}(\bar{y}, \bar{t})$. Since utility functions are strictly concave on $\mathbb{R}_{++}^{S+1}$ by Assumption 2.2.3, it holds that $\bar{u}^{i^{\prime}}(\tilde{y}, \tilde{t}) \geq u^{i^{\prime}}\left(\tilde{x}^{i^{\prime}}\right)>\bar{v}^{i^{\prime}}$, where $\tilde{x}^{i^{\prime}}=\alpha \xi^{i^{\prime}}(y, t)+(1-\alpha) \xi^{i^{\prime}}(\bar{y}, \bar{t})$ and $(\tilde{y}, \tilde{t})=\alpha(y, t)+(1-\alpha)(\bar{y}, \bar{t})$ for some $\alpha \in(0,1)$. But then $\bar{u}(\tilde{y}, \tilde{t}) \geq \bar{v}$ with strict inequality in component $i^{\prime}$. Thus, we have found an element of $V^{+}$which Pareto-dominates $\bar{v}$, a contradiction. We have shown that $\xi(y, t)=\xi(\bar{y}, \bar{t})$.

Now suppose that $y \neq \bar{y}$ and define $(\tilde{y}, \tilde{t})$ as before. By strict convexity of $Y$, there is $y^{\prime} \in Y$ such that $y^{\prime} \gg \tilde{y}$, and it follows that $\bar{u}\left(y^{\prime}, \tilde{t}\right) \gg \bar{v}$, a contradiction to $\bar{v} \in \partial V^{+}$. We have shown that $y=\bar{y}$.

For all $i \in \mathcal{I}$, we know that $\bar{u}^{i}$ is strictly increasing in the transfer, given the production plan. It follows that $t=\bar{t}$.

Theorem 4.3.7 characterizes the equilibrium payoffs of the bargaining procedure. Now, we will analyze the production and transfer decisions which lead to these payoffs. 
Definition 4.3.9 The tuple $(x, y, t, z) \in \mathbb{R}_{++}^{(S+1) I} \times B \times \mathbb{R}^{J I}$ is a bargaining equilibrium if $\bar{u}(y, t)=v^{*}$, where $v^{*} \in V^{+}$is the $\mu$-weighted Nash Bargaining Solution, and $(x, z)$ are optimal consumption bundles and asset portfolios given $(y, t)$.

The convexity of $V^{+}$implies that the $\mu$-NBS $v^{*}$ is unique. We have shown in Theorem 4.3.8 that any efficient and individually rational payoff allocation, and thus the $\mu$-weighted NBS, is supported by a unique $(y, t) \in B$, and indeed by a unique $(x, y, t, z) \in \mathbb{R}_{++}^{(S+1) I} \times B \times \mathbb{R}^{J I}$, since optimal consumption plans and portfolio choices were argued to be unique for $(y, t) \in B$. We have therefore obtained the following result.

Theorem 4.3.10 The bargaining equilibrium is unique.

\subsection{Weighted Nash Bargaining Solution}

To characterize the bargaining equilibrium, we introduce a twice differentiable quasi-convex transformation function $f: \mathbb{R}^{S+1} \rightarrow \mathbb{R}$ to describe the production possibility set. We choose $f$ such that $Y=\left\{y \in \mathbb{R}^{S+1} \mid f(y) \leq 0\right\}$. If $y \in \partial Y$, the gradient of $f(y)$ corresponds to the outward normal vector to $Y$ at $y$. It holds that $\partial f(y) \gg 0$ and $\alpha^{\top} \partial^{2} f(y) \alpha>0$ for all $\alpha \in \mathbb{R}^{S+1} \backslash\{0\}$ such that $\partial f(y) \alpha=0$.

It may be the case that the set $V^{+}$contains only the point $v^{0}$. Then, the $\mu$-weighted Nash product is equal to $v^{0}$. It follows immediately from Theorem 4.3 .8 that $y=0$ and $t=0$, but owners might still be active on the asset markets. In this case owners have nothing to gain from production by the firm, which renders the bargaining problem uninteresting. At a later stage, we will show formally that this uninteresting case can only occur degenerately.

In what follows, we will focus on the case in which $V^{+}$contains points different from $v^{0}$. In that case, Lemma 4.3.2 implies that the set $V^{++}=\left\{v \in V^{+} \mid v \gg v^{0}\right\}$ of strictly individually rational elements of $V$ is non-empty. Since all the weights $\mu$ are strictly positive, it follows that the $\mu$-NBS $v^{*}$ strictly exceeds $v^{0}$ in each component. Moreover, the set $\partial V^{++}=\partial V \cap V^{++}$of strictly individually rational and Pareto-efficient payoff allocations is parameterized by all the $\mu$-Nash Bargaining Solutions when we vary $\mu$. We proceed to derive the first-order conditions for maximizing the $\mu$-weighted Nash product.

Let $(x, y, t, z)$ be a bargaining equilibrium. It is immediate that the feasibility constraints $f(y) \leq 0$ and $\sum_{i \in \mathcal{I}} t^{i} \leq 0$ hold with equality. The optimization problem involved leads to the following first-order conditions: 


$$
\begin{array}{rlrl}
\sum_{i \in \mathcal{I}} \frac{\theta^{i} \mu^{i}}{u^{i}\left(x^{i}\right)-v^{0 i}} \partial_{x_{s}^{i}} u^{i}\left(x^{i}\right) & =\lambda \partial_{y_{s}} f(y), & s \in \mathcal{S} \\
\frac{\mu^{i}}{u^{i}\left(x^{i}\right)-v^{0 i}} \partial_{x_{0}^{i}} u^{i}\left(x^{i}\right) & =\nu, & i \in \mathcal{I}, \\
\left(\partial_{x_{0}^{i}} u^{i}\left(x^{i}\right), \partial_{x_{1}^{i}} u^{i}\left(x^{i}\right), \ldots, \partial_{x_{S}^{i}} u^{i}\left(x^{i}\right)\right) W & =0, & i \in \mathcal{I}, \\
\omega^{i}+\theta^{i} y+e(0) t^{i}+W z^{i}-x^{i} & = & 0, & i \in \mathcal{I}, \\
f(y) & =0, & \\
\sum_{i \in \mathcal{I}} t^{i} & = & &
\end{array}
$$

where $\lambda$ and $\nu$ are Lagrangian parameters corresponding to the equations specifying the feasibility of the production plan and the transfer scheme, respectively. Equation (16) corresponds to the standard conditions for optimal portfolio choice. We remark that the first-order conditions are both necessary and sufficient.

We denote the $S$-dimensional vector of marginal rates of substitution by $\nabla u^{i}\left(x^{i}\right)$, so $\nabla_{s} u^{i}\left(x^{i}\right)=\partial_{x_{s}^{i}} u^{i}\left(x^{i}\right) / \partial_{x_{0}^{i}} u^{i}\left(x^{i}\right)$ for $s=1, \ldots, S$. Similarly, we denote the $S$-dimensional vector of marginal rates of transformation by $\nabla f(y)$, so $\nabla_{s} f(y)=\partial_{y_{s}} f(y) / \partial_{y_{0}} f(y)$ for $s=1, \ldots, S$. When we substitute $\mu^{i} /\left(u^{i}\left(x^{i}\right)-v^{0 i}\right)=\nu / \partial_{x_{0}^{i}} u^{i}\left(x^{i}\right)$ in the system of equations (14), we find

$$
\begin{aligned}
\nu & =\lambda \partial_{y_{0}} f(y), \\
\sum_{i \in \mathcal{I}} \theta^{i} \nabla u^{i}\left(x^{i}\right) & =\nabla f(y) .
\end{aligned}
$$

The last equality implies that the marginal rates of transformation vector is equal to the $\theta$-weighted average of the owners' marginal rates of substitution vectors. Notice that due to potential market incompleteness, it is not guaranteed that marginal rates of substitution vectors are all equal or are equal to the marginal rate of transformation vector. Since the marginal rate of transformation vector corresponds to the outward normal vector to $Y$ at $y$, we find that $(1, \nabla f(y)) y \geq(1, \nabla f(y)) y^{\prime}$ for all $y^{\prime} \in Y$, and therefore it holds that the production plan chosen in a bargaining equilibrium maximizes

$$
y_{0}+\left(\sum_{i \in \mathcal{I}} \theta^{i} \nabla u^{i}\left(x^{i}\right)\right) y_{-0}
$$

over all production plans in $Y$. The corresponding objective function of the firm is known as the Drèze criterion. We have thereby shown the following result.

Theorem 4.4.1 In a bargaining equilibrium, the production plan is chosen according to the Drèze criterion.

For $i \in \mathcal{I}$, for $x^{i}$ with $u^{i}\left(x^{i}\right)>v^{0 i}$, define $\eta^{i}\left(x^{i}\right)=\partial_{x_{0}^{i}} u^{i}\left(x^{i}\right) /\left(u^{i}\left(x^{i}\right)-v^{0 i}\right)$. Then, (15) is equivalent to

$$
\mu^{i} \eta^{i}\left(x^{i}\right)=\nu, \quad i \in \mathcal{I}
$$


In Aumann and Kurz (1977), $\eta^{i}\left(x^{i}\right)$ is considered a measure of the owner's boldness. Consider a gamble where an owner $i$ receives utility $v^{0 i}$ with probability $p$ and $u^{i}\left(x^{i}+\varepsilon e(0)\right)$ with probability $1-p$, where $\varepsilon>0$. Let $p^{i}\left(x^{i}, \varepsilon\right)$ be the maximum probability for which owner $i$ weakly prefers the gamble over consuming $x^{i}$ for sure. As pointed out in Roth (1989), boldness corresponds to the maximum probability for which owner $i$ is willing to accept the gamble, per dollar of additional gains, when $\varepsilon$ tends to zero. That is, $\eta^{i}\left(x^{i}\right)=\lim _{\varepsilon \downarrow 0} p^{i}\left(x^{i}, \varepsilon\right) / \varepsilon$. Aumann and Kurz (1977) identify the point where boldness is equal across all owners as the Nash Bargaining Solution. The above condition says that a weighted Nash Bargaining Solution is the point where the product of boldness and probability to propose is equal for all owners. In the special case where all owners have the same probability to propose, it follows that at a bargaining equilibrium, all owners have equal boldness. Although Aumann and Kurz (1977) define boldness in a context with a single good, here we obtain a similar specification since only the marginal utility of consumption in state 0 enters $\eta^{i}\left(x^{i}\right)$.

By definition of $\nabla u^{i}\left(x^{i}\right)$, we can write Equation (16) as

$$
\nabla u^{i}\left(x^{i}\right) A=q, \quad i \in \mathcal{I} .
$$

These are simply the conditions for optimal portfolio choice for each owner. When markets are complete, for instance when $A$ is the identity, marginal rate of substitution vectors are all equal to each other and to the marginal rate of transformation vector.

The next result specifies the first-order conditions for constrained Pareto optimality and relates them to the ones corresponding to the bargaining equilibrium.

Theorem 4.4.2 It holds that $\bar{u}(y, t) \in \partial V$ if and only if $(x, y, t, z) \in \mathbb{R}_{++}^{(S+1) I} \times B \times \mathbb{R}^{J I}$ satisfies

$$
\begin{aligned}
\omega^{i}+\theta^{i} y+e(0) t^{i}+W z^{i} & =x^{i}, \quad i \in \mathcal{I}, \\
\sum_{i \in \mathcal{I}} \theta^{i} \nabla u^{i}\left(x^{i}\right) & =\nabla f(y), \\
\nabla u^{i}\left(x^{i}\right) A & =q, \quad i \in \mathcal{I}, \\
f(y) & =0, \\
\sum_{i \in \mathcal{I}} t^{i} & =0 .
\end{aligned}
$$

Moreover, $(x, y, t, z)$ is a bargaining equilibrium with $\bar{u}(y, t) \in V^{++}$if and only if it satisfies Equations (20)-(24), and

$$
\mu^{i} \eta^{i}\left(x^{i}\right)-\mu^{I} \eta^{I}\left(x^{i}\right)=0, \quad i \in \mathcal{I} \backslash\{I\}
$$

Proof: Equations (20)-(24) follow from the same derivations as in this section when applied to the maximization of the function $\prod_{i=1}^{I}\left(v^{i}\right)^{\mu^{i}}$ for arbitrarily chosen strictly positive weights $\mu^{i}$ and correspond to standard constrained Pareto optimality conditions. For a bargaining equilibrium with utilities in $V^{++}$, the equations follow from the derivations in the beginning of this section. 
The requirement $\bar{u}(y, t) \in V^{++}$is added in the second part of Theorem 4.4.2 to rule out the less interesting case where the bargaining equilibrium satisfies $\bar{u}(y, t)=v^{0}$. In that case Equations (20)-(24) are still valid, but $\eta^{i}\left(x^{i}\right)$ is not well-defined since $u^{i}\left(x^{i}\right)$ is equal to $v^{0 i}$.

We have shown that in the limit of subgame perfect equilibria in stationary strategies, the owners' bargaining procedure leads to payoffs corresponding to the $\mu$-weighted Nash Bargaining Solution. Moreover, in the previous theorem, we have translated this result on the equilibrium payoffs into a result on the production plan and transfer scheme chosen by the bargaining procedure. We will now give a formal definition of a competitive equilibrium and begin contrasting it with our findings.

Definition 4.4.3 The tuple $(x, y, t, z) \in \mathbb{R}_{++}^{(S+1) I} \times B \times \mathbb{R}^{J I}$ is a competitive equilibrium if

$$
\begin{aligned}
x^{i} & =\omega^{i}+\theta^{i} y+e(0) t^{i}+W z^{i}, & i \in \mathcal{I} \\
\sum_{i \in \mathcal{I}} \theta^{i} \nabla u^{i}\left(x^{i}\right) & =\nabla f(y), & \\
\nabla u^{i}\left(x^{i}\right) A & =q, & i \in \mathcal{I}, \\
f(y) & =0, & \\
t & =0 . &
\end{aligned}
$$

The definition of competitive equilibrium corresponds to what Magill and Quinzii (1996) define as a stock market equilibrium, when taking into account that shares are not traded. Because of this latter property, the name stock market equilibrium would be inappropriate, and we have chosen the name competitive equilibrium. We see that both the bargaining equilibrium and the competitive equilibrium require constrained Pareto-optimality, optimal choice of portfolios by all owners, and efficient production. Moreover, both notions are consistent with the Drèze criterion. The difference between both approaches is the selection made from the outcomes which satisfy constrained Pareto optimality, and which would be called pseudo equilibria or Lindahl equilibria in Drèze (1974). We next present a formal definition of such equilibria, and call them Drèze equilibria.

Definition 4.4.4 The tuple $(x, y, t, z) \in \mathbb{R}_{++}^{(S+1) I} \times B \times \mathbb{R}^{J I}$ is a Drèze equilibrium if

$$
\begin{array}{rlr}
x^{i} & =\omega^{i}+\theta^{i} y+e(0) t^{i}+W z^{i}, \quad i \in \mathcal{I}, \\
\sum_{i \in \mathcal{I}} \theta^{i} \nabla u^{i}\left(x^{i}\right) & =\nabla f(y), & \\
\nabla u^{i}\left(x^{i}\right) A & =q, & i \in \mathcal{I}, \\
f(y) & =0, & \\
\sum_{i \in \mathcal{I}} t^{i} & =0 . &
\end{array}
$$

The competitive equilibrium chooses a Drèze equilibrium allocation which does not require transfers. Bargaining power or the disagreement point play no role in this selection. Under the bargaining equilibrium, one chooses the unique allocation which can be reached by non-wasteful transfers and at which the $\mu$-weighted boldness of all owners is equal. Both the bargaining equilibrium and the competitive equilibrium are Drèze equilibria. 


\subsection{Producer Choice}

We have studied the equilibrium production and transfer decision of the firm resulting from the bargaining procedure. In the last section, we have characterized the outcome of the bargaining procedure and given a first comparison to the competitive equilibrium. In this section, we make use of the characterization given in Theorem 4.4.2 in order to study the bargaining equilibrium in much more detail and explore its relation to important concepts well-established in the literature, such as value-maximization and the competitive equilibrium.

Any matrix of security payoffs $W$ implies a set $\Pi$ of (normalized) state prices. A production plan is said to be value-maximizing if it is optimal with regard to some element of $\Pi$ (DeMarzo 1993). It turns out that the set of value-maximizing production plans is closely related to the strictly individually rational Pareto-boundary of $V$, parameterized by the weights $\mu$ of the Nash Bargaining Solution, which are in turn given by the recognition probabilities of the bargaining procedure.

Definition 4.5.1 A production plan $\bar{y} \in Y$ is value-maximizing if there is a state price vector $\pi \in \Pi$ such that for all $y \in Y$,

$$
\pi y \leq \pi \bar{y} .
$$

In what follows, we show how our previous characterization of the $\mu$-weighted NBS relates to the value-maximization concept:

Lemma 4.5.2 A production plan $\bar{y} \in Y$ is value-maximizing if and only if it satisfies

$$
\begin{aligned}
\nabla f(\bar{y}) A & =q, \\
f(\bar{y}) & =0 .
\end{aligned}
$$

Proof: This follows from the standard first-order conditions.

Theorem 4.4.2 and Lemma 4.5.2 imply that $\partial V$ is supported only by production plans which are value-maximizing. In the special case with complete markets, $\Pi$ is single-valued and value-maximization reduces to the usual profit-maximization.

Corollary 4.5.3 If $S=J$, then every $v \in \partial V$ is supported by the profit-maximizing production plan.

If markets are complete, the production decision of the firm in a bargaining equilibrium is thus consistent with the usual profit-maximizing predictions of competitive equilibrium. However, the distribution of bargaining power will determine how the firm's profits are to be divided among its owners. The bargaining equilibrium utilities may therefore differ from the competitive equilibrium utilities even if markets are complete.

For the remainder of the section, we use the notation

$$
\begin{aligned}
u_{s s^{\prime}}^{i} & =\partial^{2} u^{i}\left(x^{i}\right) / \partial x_{s}^{i} \partial x_{s^{\prime}}^{i}, \\
\nabla_{s} u^{i}\left(x^{i}\right) & =\left(\partial u^{i}\left(x^{i}\right) / \partial x_{s}^{i}\right) /\left(\partial u^{i}\left(x^{i}\right) / \partial x_{0}^{i}\right), s \in \mathcal{S} \backslash\{0\}, \\
\hat{u}_{s s^{\prime}}^{i} & =\partial\left(\nabla_{s} u^{i}\left(x^{i}\right)\right) / \partial x_{s^{\prime}}^{i} .
\end{aligned}
$$


We summarize the second-order derivatives in matrices

$$
U^{i}=\left(\begin{array}{cccc}
u_{00}^{i} & u_{01}^{i} & \cdots & u_{0 S}^{i} \\
u_{10}^{i} & u_{11}^{i} & \cdots & u_{1 S}^{i} \\
\vdots & \vdots & \ddots & \vdots \\
u_{S 0}^{i} & u_{S 1}^{i} & \cdots & u_{S S}^{i}
\end{array}\right) \text { and } \hat{U}^{i}=\left(\begin{array}{cccc}
\hat{u}_{10}^{i} & \hat{u}_{11}^{i} & \cdots & \hat{u}_{1 S}^{i} \\
\vdots & \vdots & \ddots & \vdots \\
\hat{u}_{S 0}^{i} & \hat{u}_{S 1}^{i} & \cdots & \hat{u}_{S S}^{i}
\end{array}\right) .
$$

Similarly, we write

$$
\begin{aligned}
f_{s s^{\prime}} & =\partial^{2} f(y) / \partial y_{s} \partial y_{s^{\prime}} \\
\nabla_{s} f(y) & =\left(\partial f(y) / \partial y_{s}\right) /\left(\partial f(y) / \partial y_{0}\right), s \in \mathcal{S} \backslash\{0\} \\
\hat{f}_{s s^{\prime}} & =\partial\left(\nabla_{s} f(y)\right) / \partial y_{s^{\prime}} .
\end{aligned}
$$

and use the matrices

$$
F=\left(\begin{array}{cccc}
f_{00} & f_{01} & \cdots & f_{0 S} \\
f_{10} & f_{11} & \cdots & f_{1 S} \\
\vdots & \vdots & \ddots & \vdots \\
f_{S 0} & f_{S 1} & \cdots & f_{S S}
\end{array}\right) \text { and } \hat{F}=\left(\begin{array}{cccc}
\hat{f}_{10} & \hat{f}_{11} & \cdots & \hat{f}_{1 S} \\
\vdots & \vdots & \ddots & \vdots \\
\hat{f}_{S 0} & \hat{f}_{S 1} & \cdots & \hat{f}_{S S}
\end{array}\right)
$$

Theorem 4.5.4 The set of value-maximizing production plans is an $(S-J)$-dimensional manifold.

Proof: We have to show that the matrix of partial derivatives of the following system of equations

$$
\begin{aligned}
\nabla f(y) A-q & =0, \\
f(y) & =0,
\end{aligned}
$$

has row rank $J+1$ when evaluated at a solution $y$, after which the result follows from counting equations and unknowns. To this end, we have to show that the rows of the matrix

$$
\left(\begin{array}{c}
A^{\top} \hat{F} \\
\partial f(y)
\end{array}\right)
$$

are linearly independent. We first rewrite the entry in row $j$ and column $s^{\prime}$ of $A^{\top} \hat{F}$ as follows:

$$
\begin{aligned}
{\left[A^{\top} \hat{F}\right]_{j}^{s^{\prime}} } & =\sum_{s=1}^{S} A_{s}^{j} \hat{f}_{s s^{\prime}} \\
& =\sum_{s=1}^{S} A_{s}^{j}\left(\frac{f_{s s^{\prime}} f_{0}-f_{s} f_{0 s^{\prime}}}{\left(f_{0}\right)^{2}}\right) \\
& =\frac{1}{f_{0}}\left[\sum_{s=1}^{S} A_{s}^{j} f_{s s^{\prime}}-\sum_{s=1}^{S} A_{s}^{j} \nabla_{s} f f_{0 s^{\prime}}\right] \\
& =\frac{1}{f_{0}}\left[\sum_{s=1}^{S} A_{s}^{j} f_{s s^{\prime}}-q_{j} f_{0 s^{\prime}}\right] \\
& =\left[\frac{1}{f_{0}} W^{\top} F\right]_{j}^{s^{\prime}} .
\end{aligned}
$$


The second line follows by applying the quotient rule, and the fourth line results from $\nabla f(y) A=$ $q$. Hence, we have that $A^{\top} \hat{F}=\left(1 / f_{0}\right) W^{\top} F$. Now suppose by way of contradiction that there is a row vector $\alpha \in \mathbb{R}^{J}$ and $\beta \in \mathbb{R}$ with $(\alpha, \beta) \neq 0$ such that

$$
\alpha A^{\top} \hat{F}+\beta \partial f(y)=0
$$

From right-multiplication by $W$ and $\partial f(y) W=0$, we obtain $\alpha A^{\top} \hat{F} W=0$. From rightmultiplication by $\alpha^{\top}$ and substitution of the previously derived expression for $A^{\top} \hat{F}$, we find that $\alpha W^{\top} F W \alpha^{\top}=0$. Since $f$ is differentiably quasi-convex, the last equation implies $\partial f(y) W \alpha^{\top} \neq 0$, contradicting the fact that $\partial f(y) W=0$.

We have assumed that $U^{i}$ is negative definite, implying that it is of full rank. In order to prove the next theorem, an important auxiliary result is that also the matrix of normalized second-order derivatives $\hat{U}^{i}$ has linearly independent rows. This is shown in the following lemma.

Lemma 4.5.5 The matrix $\hat{U}^{i}$ has linearly independent rows.

Proof: Suppose by way of contradiction that there is a row vector $\alpha \in \mathbb{R}^{S} \backslash\{0\}$ such that $\alpha \hat{U}^{i}=0$. By definition of $\hat{U}^{i}$ and the quotient rule

$$
\sum_{s=1}^{S} \alpha_{s}\left[\frac{u_{s s^{\prime}}^{i}}{u_{0}^{i}}-\frac{u_{s}^{i} u_{0 s^{\prime}}^{i}}{\left(u_{0}^{i}\right)^{2}}\right]=0, \quad s^{\prime}=0, \ldots, S,
$$

so we have that

$$
\sum_{s=1}^{S} \alpha_{s} u_{s s^{\prime}}^{i}-\sum_{s=1}^{S} \alpha_{s} \frac{u_{s}^{i}}{u_{0}^{i}} u_{0 s^{\prime}}^{i}=0, \quad s^{\prime}=0, \ldots, S .
$$

Now define a vector $\alpha^{\prime}=\left(-\sum_{s=1}^{S} \alpha_{s} \frac{u_{s}^{i}}{u_{0}^{i}}, \alpha_{1}, \ldots, \alpha_{S}\right)$. Then, $\sum_{s=0}^{S} \alpha_{s}^{\prime} u_{s s^{\prime}}^{i}=0$, contradicting the assumption that $U^{i}$ is negative definite.

Consider a particular $v \in \partial V$. If there exists a production plan $y \in Y$ and asset portfolios $z^{1}, \ldots, z^{I}$ such that $u^{i}\left(\omega^{i}+\theta^{i} y+W z^{i}\right)=v^{i}$ for all $i \in \mathcal{I}$, then we say that the point $v$ is supported without transfers. Notice that competitive equilibria lead to points in $\partial V$ which are supported without transfers.

In what follows, we will parameterize the economy by the initial endowments $\omega$ and the bargaining weights $\mu$. From now on, we will make this explicit by using the notation $\partial V_{\omega}$.

We state a number of results which all rely on a similar proof strategy. In each case, we phrase the problem of interest in such a way that it amounts to finding the dimension of the solution set of some system of equations. Each equation in this system can be identified with the zero of a function in which the endowment schedule $\omega$ and/or the bargaining weights $\mu$ are parameters, while the variables are production plans, transfer schemes, and asset portfolios. We summarize the relevant partial derivatives of these functions with respect to the parameters and the variables in a matrix, and prove that this matrix has linearly independent rows. The parametric transversality theorem then implies that the equations are linearly independent 
for almost all choices of the parameters. The pre-image theorem is then invoked to find the dimension of the solution set.

An important auxiliary result is that, generically in $\omega$, the disagreement point $v_{\omega}^{0}$ lies in the interior of the bargaining set.

Lemma 4.5.6 There is an open set $\Omega^{*} \subset \mathbb{R}_{++}^{(S+1) I}$ of full Lebesgue measure such that for all endowments $\omega \in \Omega^{*}$, the payoff allocation $v_{\omega}^{0}$ which arises from the optimal portfolio choice of all owners given no production and no transfers, does not belong to $\partial V_{\omega}^{+}$.

Proof: Consider the following system of equations in $\omega$ and $z$, where $\omega \in \mathbb{R}_{++}^{(S+1) I}$ and $\omega^{i}+W z^{i} \in \mathbb{R}_{++}^{S+1}$ for all $i \in \mathcal{I}$.

$$
\begin{aligned}
\sum_{i \in \mathcal{I}} \theta^{i} \nabla u^{i}\left(\omega^{i}+W z^{i}\right)-\nabla f(0) & =0 \\
\nabla u^{i}\left(\omega^{i}+W z^{i}\right) A-q & =0, i \in \mathcal{I}, \\
\nabla f(0) A-q & =0 .
\end{aligned}
$$

It holds that $v_{\omega}^{0} \in \partial V_{\omega}^{+}$if and only if (25)-(27) has a solution, as follows from the characterization of the boundary in Theorem 4.4 .2 by setting $y=0$ and $t=0$. We observe that condition (27) is independent of $\omega$. If (27) does not hold, then $v_{\omega}^{0} \notin \partial V_{\omega}^{+}$. In particular, by Assumption 2.3.3, condition (27) fails whenever markets are complete. Now consider the case where condition (27) does hold, and hence $S>J$. Consider the derivatives of conditions (25) and (26) above with respect to $\omega^{I}, \omega^{1}, \ldots, \omega^{I-1}$, which can be written in matrix form as

$$
N=\left(\begin{array}{ccccc}
\theta^{I} \hat{U}^{I} & \theta^{1} \hat{U}^{1} & \cdots & \cdots & \theta^{I-1} \hat{U}^{I-1} \\
0 & A^{\top} \hat{U}^{1} & 0 & \cdots & 0 \\
0 & 0 & \ddots & 0 & 0 \\
\vdots & \vdots & 0 & \ddots & 0 \\
0 & 0 & \cdots & 0 & A^{\top} \hat{U}^{I-1}
\end{array}\right)
$$

Lemma 4.5.5 and the full column rank of $A$ imply that the diagonal blocks $\theta^{I} \hat{U}^{I}$ and $A^{\top} \hat{U}^{i}, i=1, \ldots, I-1$, have linearly independent rows. Therefore, the rows of $N$ are independent. By the parametric transversality theorem, this implies that the derivatives of the aforementioned expressions with respect to $z$ alone are linearly independent for almost all $\omega \in \mathbb{R}_{++}^{(S+1) I}$. Applying the pre-image theorem and counting unknowns and equations, we find that the set of solutions to the above system is a manifold of dimension $J-S<0$ for almost all $\omega$. We have shown that the set $\Omega^{*}$ of endowments $\omega$ such that $v_{\omega}^{0} \notin \partial V_{\omega}^{+}$is of full Lebesgue measure. It holds that $v_{\omega}^{0}$ is a continuous function of $\omega$, and $\partial V_{\omega}^{+}$is an upper-hemi-continuous correspondence in $\omega$. Hence, if $v_{\bar{\omega}}^{0} \notin \partial V_{\bar{\omega}}^{+}$for some particular $\bar{\omega} \in \Omega^{*}$, then $v_{\omega}^{0} \notin \partial V_{\omega}^{+}$for all $\omega$ in a sufficiently small neighborhood of $\bar{\omega}$. Therefore, $\Omega^{*}$ is open, and the lemma follows.

Theorem 4.5.7 There is an open set $P^{*} \subset \mathbb{R}_{++}^{(S+1) I} \times \Delta^{I}$ of full Lebesgue measure such that for all $(\omega, \mu) \in P^{*}$, the bargaining equilibrium involves non-zero transfers. 
Proof: We will introduce a system of equations in $\omega, \mu, y, z$, and $\bar{z}$, where the variables are restricted to satisfy the following conditions.

$$
\begin{aligned}
\mu^{i} & >0, i \in \mathcal{I}, \\
\omega^{i} & \gg 0, i \in \mathcal{I}, \\
\omega^{i}+\theta^{i} y+W z^{i} & \gg 0, i \in \mathcal{I}, \\
\omega^{i}+W \bar{z}^{i} & \gg 0, i \in \mathcal{I}, \\
u^{i}\left(\omega^{i}+\theta^{i} y+W z^{i}\right)-u^{i}\left(\omega^{i}+W \bar{z}^{i}\right) & >0, i \in \mathcal{I} .
\end{aligned}
$$

The set of $(\omega, \mu, y, z, \bar{z})$ satisfying these restrictions is open, and therefore a manifold. The system of equations is as follows.

$$
\begin{aligned}
\nabla f(y) A-q & =0, \\
f(y) & =0, \\
\nabla u^{i}\left(\omega^{i}+\theta^{i} y+W z^{i}\right) A-q & =0, i \in \mathcal{I} \backslash\{I\}, \\
\sum_{i \in \mathcal{I}} \theta^{i} \nabla u^{i}\left(\omega^{i}+\theta^{i} y+W z^{i}\right)-\nabla f(y) & =0, \\
\gamma^{1}\left(\omega^{1}, \mu^{1}, y, z^{1}, \bar{z}^{1}\right)-\gamma^{I}\left(\omega^{I}, \mu^{I}, y, z^{I}, \bar{z}^{I}\right) & =0, \\
\nabla u^{i}\left(\omega^{i}+W \bar{z}^{i}\right) A-q & =0, i \in \mathcal{I},
\end{aligned}
$$

where we have defined

$$
\gamma^{i}\left(\omega^{i}, \mu^{i}, y, z^{i}, \bar{z}^{i}\right)=\frac{u^{i}\left(\omega^{i}+\theta^{i} y+W z^{i}\right)-u^{i}\left(\omega^{i}+W \bar{z}^{i}\right)}{\mu^{i} \partial_{0} u^{i}\left(\omega^{i}+\theta^{i} y+W z^{i}\right)}, i \in \mathcal{I} .
$$

Given $(\omega, \mu)$, the solution to this system is a tuple $(y, z, \bar{z})$ such that the production plan $y$, the asset portfolios $z$, and zero transfers correspond to a $\mu$-bargaining equilibrium with $v_{\omega}^{0} \in \partial V_{\omega}^{+}$and, moreover, $\bar{z}$ are asset portfolios corresponding to the disagreement point. We want to show that for generically chosen $(\omega, \mu)$, this system is over-determined. This amounts to proving that the derivatives of the above equations are linearly independent.

Consider a block matrix $M$ in which the first row corresponds to the $J+1$ derivatives of equations (28)-(29), the second row to the $I J+S-J+1$ derivatives of equations (30)-(32), and the third row to the $I J$ derivatives of equation (33). Moreover, the first column gives the derivatives with respect to $y$, the second column with respect to $(\omega, \mu)$, and the third column with respect to $\bar{z}$. The proof strategy is to show that $M$ is of the lower-triangular form,

$$
M=\left(\begin{array}{ccc}
* & 0 & 0 \\
& * & 0 \\
& & *
\end{array}\right)
$$

where the diagonal blocks have rank $J+1, I J+S-J+1$, and $I J$, respectively.

The proof of Theorem 4.5.4 shows that the first diagonal block is of rank $J+1$. The corresponding equations do not involve $(\omega, \mu)$ nor $\bar{z}$. This explains the form of the first row of $M$. 
Now consider the last diagonal block of $M$. It corresponds to the block-diagonal matrix

$$
M^{33}=\left(\begin{array}{cccc}
A^{\top} \hat{U}^{1} W & 0 & \cdots & 0 \\
0 & \ddots & & \vdots \\
\vdots & & \ddots & 0 \\
0 & \cdots & 0 & A^{\top} \hat{U}^{I} W
\end{array}\right)
$$

Each block of the matrix above is of rank $J$, reflecting the fact that there is a unique optimal asset portfolio for each agent, given the endowments, production decision, and transfers (which are zero in the case at hand).

We now proceed to the second row of $M$. We argue first that the derivatives of equations (30)-(32) with respect to $\bar{z}$ are all zero. This is obvious for equations (30)-(31) which do not involve $\bar{z}$. With regard to equation (32), the derivative of any $\gamma^{i}$ with respect to $\bar{z}^{i}$ is of the form $\kappa \partial u^{i}\left(\omega^{i}+W \bar{z}^{i}\right) W$, where $\kappa$ is a scalar. But at the solution to the equation system above, $\bar{z}^{i}$ is chosen optimally as by equation (33), so that $\partial u^{i}\left(\omega^{i}+W \bar{z}^{i}\right) W=0$, as desired.

Now it remains to show that the block in the center of the matrix $M$ is of full rank. It corresponds to the derivatives of equations (30)-(32) with respect to $(\omega, \mu)$. Omitting the derivatives with respect to $\mu^{i}$ for $i=1, \ldots, I-1$, it can be spelled out as follows.

$$
M^{22}=\left(\begin{array}{cccccc}
A^{\top} \hat{U}^{1} & 0 & \cdots & \cdots & 0 & 0 \\
0 & \ddots & & & \vdots & \vdots \\
\vdots & & \ddots & & 0 & 0 \\
0 & \cdots & \cdots & A^{\top} \hat{U}^{I-1} & 0 & 0 \\
\theta^{1} \hat{U}^{1} & \cdots & \cdots & \cdots & \theta^{I} \hat{U}^{I} & 0 \\
\partial_{\omega^{1}} \gamma^{1} & 0 & \cdots & 0 & \partial_{\omega^{I}} \gamma^{I} & \partial_{\mu^{I}} \gamma^{I}
\end{array}\right)
$$

The linear independence of the first $(I-1) J+S-J$ rows of $M^{22}$ has been shown in the proof of Lemma 4.5.6. We can write $\partial_{\mu^{I}} \gamma^{I}=-\kappa^{I}\left(\mu^{I}\right)^{(-2)}$, where the factor $\kappa^{I}$ is strictly positive by the restriction that $u^{i}\left(\omega^{i}+\theta^{i} y+W z^{i}\right)>u^{i}\left(\omega^{i}+W \bar{z}^{i}\right)$ for all $i \in \mathcal{I}$. Also, we have assumed $\mu \gg 0$, thus $\partial_{\mu^{I}} \gamma^{I}<0$, as required.

We have now shown that the rows of $M$ are linearly independent. By the parametric transversality theorem, we can conclude that the set of $(\omega, \mu) \in \mathbb{R}_{++}^{(S+1) I} \times \Delta^{I}$ for which the derivatives with respect to the remaining variables are linearly independent is of full Lebesgue measure. By the preimage theorem, we may then count equations and unknowns and find that the set of $(\omega, \mu) \in \mathbb{R}_{++}^{(S+1) I} \times \Delta^{I}$ for which the equation system is over-determined is of full Lebesgue measure. Denote the intersection of that set with $\Omega^{*}$ by $P^{*}$. If $(\omega, \mu) \in P^{*}$, then the bargaining equilibrium involves non-zero transfers. The set $P^{*}$ is of full Lebesgue measure. Moreover, the (unique) bargaining equilibrium is a continuous function of $(\omega, \mu)$. Therefore, if the property of non-zero transfers holds for some $(\bar{\omega}, \bar{\mu}) \in \mathbb{R}_{++}^{(S+1) I} \times \Delta^{I}$, then it also holds for $(\omega, \mu) \in \mathbb{R}_{++}^{(S+1) I} \times \Delta^{I}$ in a sufficiently small neighborhood of $(\bar{\omega}, \bar{\mu})$. Hence, the set $P^{*}$ is open.

Theorem 4.5.7 says that generically in endowments and bargaining weights the bargaining 
procedure will lead to transfers. Since competitive equilibria involve zero transfers, bargaining equilibria are generically distinct from competitive equilibria.

Corollary 4.5.8 There is an open set $P^{*} \subset \mathbb{R}_{++}^{(S+1) I} \times \Delta^{I}$ of full Lebesgue measure such that for all $(\omega, \mu) \in P^{*}$, the bargaining equilibrium is not a competitive equilibrium.

Since by Theorem 4.3.8 points in $\partial V^{+}$are supported by uniquely defined production plans and transfer schemes, the bargaining equilibrium utilities and the competitive equilibrium utilities are different for almost all endowments and bargaining weights.

Corollary 4.5.9 There is an open set $P^{*} \subset \mathbb{R}_{++}^{(S+1) I} \times \Delta^{I}$ of full Lebesgue measure such that for all $(\omega, \mu) \in P^{*}$, the bargaining equilibrium utilities are not equal to competitive equilibrium utilities.

This difference in payoff allocation holds for both complete and incomplete markets. In the case of complete markets, we have previously shown that the profit-maximizing production plan is selected by the bargaining procedure. Thus, with complete markets, any difference in payoff allocation must be due to the transfers. With regard to incomplete markets, however, we will show in the sequel of this section that the difference in payoff allocation is not only the result of transfers, but that the chosen production plan is different as well.

Theorem 4.5.10 Suppose that markets are incomplete, that is, $J<S$. Then, there is an open set $P^{* *} \subset \mathbb{R}_{++}^{(S+1) I} \times \Delta^{I}$ of full Lebesgue measure such that for all $(\omega, \mu) \in P^{* *}$, the bargaining equilibrium production plan is not a competitive equilibrium production plan.

Proof: We introduce a system of equations in $(\omega, \mu), y, t, z, \hat{z}$, and $\bar{z}$, where the variables are restricted as follows.

$$
\begin{aligned}
\mu^{i} & >0, i \in \mathcal{I}, \\
\omega^{i} & \gg 0, i \in \mathcal{I}, \\
\omega^{i}+\theta^{i} y+e(0) t^{i}+W z^{i} & \gg 0, i \in \mathcal{I}, \\
\omega^{i}+\theta^{i} y+W \hat{z}^{i} & \gg 0, i \in \mathcal{I}, \\
\omega^{i}+W \bar{z}^{i} & \gg 0, i \in \mathcal{I}, \\
u^{i}\left(\omega^{i}+\theta^{i} y+e(0) t^{i}+W z^{i}\right)-u^{i}\left(\omega^{i}+W \bar{z}^{i}\right) & >0, i \in \mathcal{I} .
\end{aligned}
$$

The set of variables satisfying these restrictions is open, and is therefore a manifold. The system of equations under consideration is as follows. 


$$
\begin{aligned}
\sum_{i \in \mathcal{I}} t^{i} & =0, \\
\nabla f(y) A-q & =0, \\
f(y) & =0, \\
\nabla u^{i}\left(\omega^{i}+\theta^{i} y+e(0) t^{i}+W z^{i}\right) A-q & =0, i \in \mathcal{I} \backslash\{I\}, \\
\sum_{i \in \mathcal{I}} \theta^{i} \nabla u^{i}\left(\omega^{i}+\theta^{i} y+e(0) t^{i}+W z^{i}\right)-\nabla f(y) & =0, \\
\gamma^{i}\left(\omega^{i}, \mu^{i}, y, t^{i}, z^{i}, \bar{z}^{i}\right)-\gamma^{I}\left(\omega^{I}, \mu^{I}, y, t^{I}, z^{I}, \bar{z}^{I}\right) & =0, i \in \mathcal{I} \backslash\{I\}, \\
\nabla u^{i}\left(\omega^{i}+\theta^{i} y+W \hat{z}^{i}\right) A-q & =0, i \in \mathcal{I} \backslash\{I\}, \\
\sum_{i \in \mathcal{I}} \theta^{i} \nabla u^{i}\left(\omega^{i}+\theta^{i} y+W \hat{z}^{i}\right)-\nabla f(y) & =0, \\
A^{\top} \nabla u^{i}\left(\omega^{i}+W \bar{z}^{i}\right)-q & =0, i \in \mathcal{I} .
\end{aligned}
$$

In words, the equations specify that -given $(\omega, \mu)-(y, t, z)$ is the $\mu$-bargaining equilibrium, and $(y, 0, \hat{z})$ is the competitive equilibrium, where for $i \in \mathcal{I}$, we have $x^{i}=\omega^{i}+\theta^{i} y+e(0) t^{i}+W z^{i}$ and $\hat{x}^{i}=\omega^{i}+\theta^{i} y+W \hat{z}^{i}$. We want to show that for generically chosen $(\omega, \mu)$, this system is overdetermined whenever markets are complete. This is equivalent to proving that the equations in the system above are all linearly independent.

Let $N$ be a matrix where the first row corresponds to the derivative of equation (34), the second row corresponds to the $J+1$ derivatives of equations (35)-(36), the third row corresponds to $I J+S-J+I-1$ derivatives of equations (37)-(39), the fourth row to the $I J+S-J$ derivatives of equations (40)-(41) and the fifth row corresponds to the $I J$ derivatives of equation (42). Moreover, the first column refers to derivatives with respect to $t$, the second column with respect to $y$, the third column with respect to $(\omega, \mu)$, the fourth column with respect to $\hat{z}$, and the fifth column with respect to $\bar{z}$.

We will show that $N$ is of the lower triangular form,

$$
N=\left(\begin{array}{ccccc}
* & 0 & 0 & 0 & 0 \\
& * & 0 & 0 & 0 \\
& & * & 0 & 0 \\
& & & * & 0 \\
& & & & *
\end{array}\right),
$$

where the diagonal blocks are of full row rank $1, J+1, I J+S-J+I-1, I J+S-J$, and $I J$, respectively. By the parametric transversality theorem, this means that for generic $(\omega, \mu)$, the matrix of derivatives with respect to the remaining variables $(y, z, \hat{z}, \bar{z}, t)$ has linearly independent rows. Applying the pre-image theorem, this will imply that the set of $(y, z, \hat{z}, \bar{z}, t)$ which solve the above equation system given a generic $(\omega, \mu)$ is a manifold of dimension $J-S$, and corresponds therefore to the empty set when $J<S$.

Equation (34) involves only $t$, which explains the form of the first row of $N$. Equations (35)-(36) involve only $y$. This together with Theorem 4.5.4 explains that the second row of $N$ 
is of the form indicated above. The linear independence of the $I J$ rows in the last diagonal block of $N$ follows by the same argument as in the proof of Theorem 4.5.7. Aside from equation (42), the portfolios $\bar{z}$ are only involved in equation (39). But the derivative of (39) with respect to $\bar{z}$ must be zero at the solution to the equation system, whence the zero entries in the fifth column of $N$ - the argument is as in the proof of Theorem 4.5.7. Similarly, the portfolios $\hat{z}$ are involved only in equations (40)-(41), explaining the zero entries in the fourth column of $N$.

Consider the third diagonal block of $N$. After appropriate permutations of the rows and columns, it can be spelled out as follows.

$$
N^{33}=\left(\begin{array}{cccccccccc}
A^{\top} \hat{U}^{1} & 0 & \cdots & \cdots & 0 & 0 & 0 & 0 & 0 & 0 \\
0 & \ddots & & & \vdots & \vdots & \vdots & \vdots & \vdots & \vdots \\
\vdots & & \ddots & & 0 & \vdots & \vdots & \vdots & \vdots & \vdots \\
0 & \cdots & \cdots & A^{\top} \hat{U}^{I-1} & 0 & \vdots & \vdots & \vdots & \vdots & \vdots \\
\theta^{1} \hat{U}^{1} & \cdots & \cdots & \cdots & \theta^{I} \hat{U}^{I} & 0 & 0 & 0 & 0 & 0 \\
\partial_{\omega^{1}} \gamma^{1} & 0 & \cdots & 0 & \partial_{\omega^{I}} \gamma^{I} & \partial_{\mu^{1}} \gamma^{1} & 0 & \cdots & 0 & \partial_{\mu^{I}} \gamma^{I} \\
0 & \ddots & & & \vdots & 0 & \ddots & & \vdots & \vdots \\
\vdots & & \ddots & & \vdots & \vdots & & \ddots & 0 & \vdots \\
0 & \cdots & 0 & \partial_{\omega^{I-1}} \gamma^{I-1} & \partial_{\omega^{I}} \gamma^{I} & 0 & \cdots & 0 & \partial_{\mu^{I-1}} \gamma^{I-1} & \partial_{\mu^{I}} \gamma^{I}
\end{array}\right)
$$

The block in the upper left corner has previously been shown to be of rank $I J+S-J$, see the proof of Lemma 4.5.6. We have argued in the proof of Theorem 4.5.7 that the terms of the form $\partial_{\mu^{i}} \gamma^{i}$ are strictly positive, which readily implies the full rank $I-1$ of the block in the lower right corner. Let $P^{* *}$ be the intersection of $\Omega^{*}$ with the set of all $(\omega, \mu) \in \mathbb{R}_{++}^{(S+1) I} \times \Delta^{I}$ for which the matrix under consideration has linearly independent rows. We have shown that $P^{* *}$ is of full Lebesgue measure. The bargaining equilibrium is a continuous function of $(\omega, \mu)$, and the competitive equilibria are an upper-hemi-continuous correspondence of $(\omega, \mu)$. If the property of strictly positive distance between the bargaining and competitive equilibrium production plans holds for some $(\bar{\omega}, \bar{\mu}) \in \mathbb{R}_{++}^{(S+1) I} \times \Delta^{I}$, then it is preserved for $(\omega, \mu) \in \mathbb{R}_{++}^{(S+1) I} \times \Delta^{I}$ in a sufficiently small neighborhood around $(\bar{\omega}, \bar{\mu})$.

We had previously shown that generically in endowments and bargaining weights the payoff allocation resulting from the bargaining equilibrium and that resulting from a competitive equilibrium are different. We have seen that in the case of complete markets, the production plan chosen under both approaches is the same, so that the different payoff allocation is merely a result of redistribution via transfers. The last theorem complements these findings by saying that for the case of incomplete markets, the two approaches almost always lead to different production plans.

\subsection{Conclusion}

We have introduced a non-cooperative bargaining procedure to resolve the conflict among shareholders of a firm when markets are incomplete. In contrast to many existing models, we 
obtain a unique prediction for the production plan as well as for the resulting payoff allocation. This solution is parameterized by the distribution of bargaining power across the different owners. This bargaining power distribution is independent of the shares of ownership.

An important feature of the model is that transfers are possible in equilibrium. The wellknown Drèze criterion rules out a production plan which can be Pareto-improved upon by an alternative plan and transfers. The well-known solution concept of a stock market equilibrium satisfies this criterion but requires that the chosen production plan itself should be implemented without transfers. Our solution concept, called a bargaining equilibrium, satisfies the Drèze criterion but does allow for transfers to be made in equilibrium. Indeed, it turns out that transfers will almost always be used. Furthermore, the bargaining equilibrium is derived from an explicit non-cooperative bargaining model. The outcome of the bargaining procedure proposed in this paper is different from the predictions of standard economic theory. If markets are complete, the production decision of the firm is driven by profit-maximization as in the Arrow-Debreu model. However, the profits are redistributed among the owners of the firm in accordance with their bargaining power, which derives from the ability to make a proposal and from the disagreement payoff. In the case of incomplete markets, the production plan adopted under the bargaining procedure almost always fails to be a competitive equilibrium production plan. Non-zero transfers are almost always made. We have given positive support for the use of the Drèze criterion, though the utility gradients of owners implied by our theory differ from the competitive equilibrium ones.

In our bargaining game we have not considered the option for owners to modify ownership shares and/or to sell the firm to outsiders. An intriguing question for future research is whether allowing such possibilities would give support for the criterion proposed by Bisin, Gottardi and Ruta (2009), which loosely speaking corresponds to the maximal utility gradient in the population rather than a weighted average of the owner's utility gradients as a criterion. 



\section{Summary}

This dissertation has addressed the problem of dividing gains from cooperation through a bargaining process. It has discussed two models where such gains from cooperation are abstract and one where they result from a particular economic situation, namely the cooperation among different owners of a firm. In each of the three models under consideration, a bargaining process in the spirit of Rubinstein is used as the mechanism by which agreement on the payoff allocation is found.

Chapter 2 has focussed on the Rubinstein bargaining process itself and, more in particular, on the relationship between the proposer selection rule and the equilibrium outcome in the limit as bargaining friction goes to zero. The chapter concludes that a player's relative bargaining power converges to the fraction of the time that this player expects to be the proposer in the long run.

Chapter 3 considers a setup where such a Rubinstein bargaining process (now with two players only) is preceded by a commitment stage. In that stage, players can make binding commitments not to enter into certain agreements. Their ability to do so is subject to two straight-forward restrictions. A commitment must be expressed in the terms which the player derives utility from, and a player cannot remain committed to something which has become impossible. In this setup, it turns out that there is an intermediate optimal level of commitment; that is, a player does not optimally choose to commit to the entire surplus. Moreover, Chapter 3 contains a result which suggests that the equal split is a uniquely robust focal point within a range of surplus divisions which can be supported by equilibria.

Chapter 4 applies bargaining theory to the conflict of interest which tend to arise among the owners of a firm when financial markets are incomplete, thereby contributing a new approach to the literature on the theory of the firm. The decision-making problem within a firm has typically been looked at from a normative perspective, and criteria to resolve the conflict have not so far been based on explicit bargaining foundations. Chapter 4 aims to begin filling this gap in the literature. 



\section{Nederlandse Samenvatting}

In dit proefschrift wordt onderzocht hoe spelers door een onderhandelingsproces een akkoord bereiken over de verdeling van winsten uit samenwerking. Het proefschrift omvat twee modellen waarin die winst abstract is en één waarin die winst voortvloeit uit een specifieke vorm van samenwerking, namelijk die tussen de verschillende aandeelhouders van een bedrijf. Er worden methoden uit de (micro-)economische theorie en in het bijzonder uit de speltheorie toegepast.

Hoofdstuk 2 richt zich op het onderhandelingsproces zelf en op de uitbetalingen van de spelers in een evenwicht van zo'n onderhandelingsspel. In elke onderhandelingsronde heeft een van de spelers het recht om een voorstel te doen waarmee iedereen zich vervolgens wel of niet akkoord verklaart. Er zijn regels die bepalen wanneer welke speler dit recht heeft. Die regels worden ook het protocol genoemd en vormen een belangrijke bron van onderhandelingsmacht. De uitbetaling van een speler is dan ook gekoppeld aan de fractie van de tijd waarin hij op lange termijn verwacht voorstellen te kunnen doen.

Hoofdstuk 3 behandelt een andere bron van onderhandelingsmacht, namelijk commitment. Er is sprake van commitment wanneer een speler voorafgaand aan de onderhandeling bepaalde opties geloofwaardig kan uitsluiten. Bijvoorbeeld zou de speler minimale eisen kunnen stellen en beloven dat hij nooit met een voorstel zal instemmen die daar niet aan voldoet. In hoofdstuk 3 wordt verondersteld dat de geloofwaardigheid van een commitment in twee opzichten beperkt is: De speler mag niets eisen waar hij niet om geeft, en de speler mag niets blijven eisen wat onmogelijk is geworden. Die modellering van commitment is nieuw in de literatuur en leidt tot inzichten over de optimale keuze van commitments.

In hoofdstuk 4 worden inzichten uit onderhandelingsspelen toegepast op de besluitvorming in een bedrijf met meerdere aandeelhouders. Als hun gemeenschappelijk bedrijf een onzekere toekomst tegemoet gaat willen de aandeelhouders zich tegen die onzekerheid beschermen door een verstandige individuele keuze van beleggingen. Als echter de financiële markten hun hiertoe onvoldoende mogelijkheden bieden (incomplete markets) dan ontstaat er een conflict tussen de aandeelhouders. Dit conflict wordt in de literatuur meestal normatief benadert. Hoofdstuk 4 voegt hier op basis van de speltheorie een positieve benadering aan toe. 



\section{References}

Abreu, D. And F. Gul (2000), "Bargaining and reputation," Econometrica, 68, 85-117.

Aumann, R.J., And M. Kurz (1977), "Power and Taxes," Econometrica, 45, 1137-1161.

Banks, J. And J. Duggan (2000), "A Bargaining Model of Collective Choice," American Political Science Review, 94, 73-88.

Bester, H. (1993), "Bargaining versus Price Competition in Markets with Quality Unvertainty," The American Economic Review, 83 (1993), 278-288.

Binmore, K.G. (1987), "Perfect Equilibria in Bargaining Models," in K.Binmore and P.Dasgupta (Eds.), The Economics of Bargaining, Blackwell, Oxford.

Binmore, K., A. Rubinstein, And A. Wolinsky (1986), "The Nash Bargaining Solution in Economic Modelling," The RAND Journal of Economics, 17, 176-188.

Bisin, A., P. Gottardi, and G. Ruta (2009), "Equilibrium Corporate Finance," Working Paper, European University Institute.

Bonnisseau, J.-M., And O. Lachiri (2004), "On the Objective of Firms under Uncertainty with Stock Markets," Journal of Mathematical Economics, 40, 493-513.

Britz, V. (2010), "Optimal Value Commitment in Bilateral Bargaining," METEOR Research Memorandum RM/10/056.

Britz, V., P.J.J. Herings, and A. Predtetchinski (2010), "Non-cooperative Support for the Asymmetric Nash Bargaining Solution," Journal of Economic Theory, 145, 1951-1967.

Britz, V., P.J.J. Herings, and A.Predtetchinski (2010), "Theory of the Firm - Bargaining and Competitive Equilibrium," METEOR Research Memorandum RM/10/057.

Calabuig, V., A. Cunyat, and G. Olcina (2002), "Commitment and choice of partner in a negotiation with a deadline," Spanish Economic Review, 4, 61-87.

Carlsson, H. (1991), "A Bargaining Model Where Parties Make Errors," Econometrica, 59, 14871496.

Chae, S. And J.A. Yang (1994), "An N-Person Pure Bargaining Game," Journal of Economic Theory, 62 (1994), 86-102.

Cho, S.J., And J. Duggan (2005), "Bargaining Foundations of the Median Voter Theorem," Working Paper, 1-32.

Cunyat, A. (2004), "The optimal degree of commitment in a negotiation with a deadline," Economic Theory, 23, 455-465.

DeMarzo, P. (1993), "Majority Voting and Corporate Control: The Rule of the Dominant Shareholder," Review of Economic Studies, 60, 713-734.

Diamond, P.A. (1967), "The Role of a Stock Market in a General Equilibrium Model with Technological Uncertainty," American Economic Review, 57, 759-776.

Dierker, E., H. Dierker, And B. Grodal (2002), "Nonexistence of Constrained Efficient Equilibria When Markets Are Incomplete," Econometrica 70, 1245-1251.

Drèze, J.H. (1974), "Investment under Private Ownership: Optimality, Equilibrium, and Stability," in J.H. Drèze, ed., Allocation under Uncertainty: Equilibrium and Optimality (New York: Macmillan, 1974) 9. 
Drèze, J.H., O. Lachiri, And E. Mineldi (2009), "Stock Prices, Anticipations and Investment in General Equilibrium," CORE Discussion Paper, 2009/83, 1-46.

Fershtman, C. and D. Seidmann (1993), "Deadline effects and inefficient delay in bargaining with endogenous commitment," Journal of Economic Theory, 60, 306-321.

Grossman, S.J., and O. Hart (1979), "A Theory of Competitive Equilibrium in Stock Market Economies," Econometrica, 47, 293-330.

Grout, P.A. (1984), "Investment and Wages in the Absence of Binding Contracts: A Nash Bargaining Approach," Econometrica, 52 , 449-460.

Harsanyi, J.C. and R. Selten (1988), A General Theory of Equilibrium Selection in Games, MIT Press, Cambridge.

Hart, S. And A. Mas-Colell (1996), "Bargaining and Value," Econometrica, 64, 357-380.

Herings, P.J.J., And A. Predtetchinski (2009), "One-dimensional Bargaining with Markov Recognition Probabilities," Journal of Economic Theory, doi:10.1016/j.jet.2009.10.002.

Hyndman, K., And D. Ray (2007), "Coalition Formation with Binding Agreements," Review of Economic Studies, 74, 1125-1147.

Kalai, E. (1977), "Nonsymmetric Nash Solutions and Replications of 2-Person Bargaining," International Journal of Game Theory, 6, 129-133.

Kalandrakis, T. (2004), "Equilibria in Sequential Bargaining Games as Solutions to Systems of Equations," Economics Letters, 84, 407-411.

KamBe, S. (1999), "Bargaining with imperfect commitment," Games and Economic Behavior, 28, 217-237.

Kelsey, D., And F. Milne (1996), "The Existence of Equilibrium in Incomplete Markets and the Objective Function of the Firm," Journal of Mathematical Economics, 25, 229-245.

Krishna, V., And R. Serrano (1996), "Multilateral Bargaining," The Review of Economic Studies, 63, 61-80.

Kultti, K., And H. Vartiainen (2007), "Multilateral Non-Cooperative Bargaining in a General Utility Space," Working Paper, downloadable from http://personal.inet.fi/tiede/yjs-hv.

Laruelle, A., and F.Valenciano (2007), "Bargaining in Committees as an Extension of Nash's Bargaining Theory," Journal of Economic Theory, 132, 291-305.

Laruelle, A., And F. Valenciano (2008), "Noncooperative Foundations of Bargaining Power in Committees and the Shapley-Shubik Index," Games and Economic Behavior, 63, 341-353.

Lensberg, T. (1988), "Stability and the Nash Solution," Journal of Economic Theory, 45, 330-341.

LI, D. (2007), "Bargaining with history-dependent preferences," Journal of Economic Theory, 136, 695-708.

Magill, M., And M. QuinziI (1996), Theory of Incomplete Markets, MIT Press, Cambridge, Massachusetts.

Merlo, A., And C. Wilson (1995), "A Stochastic Model of Sequential Bargaining with Complete Information," Econometrica, 63, 371-399.

MryakaWa, T. (2008), "Noncooperative Foundation of $n$-Person Asymmetric Nash Bargaining Solution," Journal of Economics of Kwansei Gakuin University, 62, 1-18. 
Muthoo, A. (1992), "Revocable commitment and sequential bargaining," The Economic Journal, 102, 378-387.

Muthoo, A. (1996), "A bargaining model based on the commitment tactic," Journal of Economic Theory, 69, 134-152

NAsh, J.F. (1950), "The Bargaining Problem," Econometrica, 18, 155-162.

Nash, J.F. (1953), "Two-Person Cooperative Games," Econometrica, 21, 128-140.

Rockafellar, R.T. (1970), Convex Analysis, Princeton University Press, Princeton, New Jersey.

Roth, A.E. (1989), "Risk Aversion and the Relationship between Nash's Solution and Subgame Perfect Equilibrium of Sequential Bargaining," Journal of Risk and Uncertainty, 2, 353-365.

Rubinstein, A.(1982), "Perfect equilibrium in a bargaining model," Econometrica, 50, 97-109.

Schelling, T.C. (1956), "An essay on bargaining," American Economic Review, 46, 281-306.

Schelling, T.C. (1960), "The Strategy of Conflict," Cambridge: Harvard University Press.

Shaked, A. And J. Sutton (1984), "Involuntary Unemployment as a Perfect Equilibrium in a Bargaining Model," Econometrica, 52, 1351-1364.

Tvede, M., ANd H. CRes (2005), "Voting in Assemblies of Shareholders and Incomplete Markets," Economic Theory, 26, 887-906. 



\section{Short Curriculum Vitae}

Volker Britz was born on May 26, 1982 in Bonn, Germany. He attended the Rurtal-Gymnasium in Düren for secondary school and graduated in 2001. Afterwards, he studied Economics at Maastricht University and graduated in 2005 with the B.Sc. degree in the graduate option International Economic Studies. In 2006, Volker obtained his M.Sc. degree in Economics from Maastricht University. As a M.Sc. student, he focussed on growth theory and wrote his thesis on the resource curse. Afterwards, his main interests have been in microeconomic theory and, in particular, game theory. From 2006 until 2011, Volker prepared his dissertation under

the supervision of Prof. Dr. P. Jean-Jacques Herings and Dr. Arkadi Predtetchinski in the Department of Economics, Maastricht University. 